Portland State University

PDXScholar

Spring 6-3-2015

\title{
Transformational Leadership, Diversity, and Creativity at Work: A Moderated Mediation Model
}

Aisha Smith Taylor

Portland State University

Follow this and additional works at: https://pdxscholar.library.pdx.edu/open_access_etds

Part of the Industrial and Organizational Psychology Commons

Let us know how access to this document benefits you.

\section{Recommended Citation}

Taylor, Aisha Smith, "Transformational Leadership, Diversity, and Creativity at Work: A Moderated Mediation Model" (2015). Dissertations and Theses. Paper 2341.

https://doi.org/10.15760/etd.2338

This Dissertation is brought to you for free and open access. It has been accepted for inclusion in Dissertations and Theses by an authorized administrator of PDXScholar. For more information, please contact pdxscholar@pdx.edu. 
Transformational Leadership, Diversity, and Creativity at Work:

A Moderated Mediation Model

by

Aisha Smith Taylor

A dissertation submitted in partial fulfillment of the requirements for the degree of

\author{
Doctor of Philosophy \\ in \\ Applied Psychology
}
Dissertation Committee:
Keith James, Chair
Todd Bodner
Sully Taylor
Liu-Qin Yang

Portland State University

2015 
(C) 2015 Aisha Smith Taylor 


\begin{abstract}
Organizational leaders often seek to hire and retain innovative employees as a source of competitive advantage. Both transformational leadership and effectively managed workplace diversity have been theorized and shown to lead to increased employee creative performance at work; however, a full model of the relationships between leadership and the multi-dimensional construct of workplace diversity has not yet been tested. Using a sample of 371 employees in three Chinese high-technology firms matched with 64 supervisors collected at three time points, this study theorized and tested a moderated mediation path model in which transformational leadership and diversity climate were predicted to significantly interact to influence the workplace diversity constructs of organizational justice and organizational identity, which in turn, influence individual creative performance. Based on major theories of leadership, diversity, and creativity, several partial mediation hypotheses are presented, including diversity climate as a mediator of the relationship between transformational leadership and creative performance as well as organizational justice and organizational identity as mediators of the relationship between the interaction of transformational leadership and diversity climate and creative performance. Several single- and multilevel path analyses were conducted to test the model, using two measures of creative performance: self-ratings and supervisor ratings. The results showed that the interaction of transformational leadership and diversity climate significantly predicted self-rated creative performance, and organizational identity significantly predicted supervisor ratings of creative performance. In addition, transformational leadership was found to significantly predict diversity
\end{abstract}


climate and organizational justice was a significant predictor of organizational identity.

Finally, transformational leadership had a significant indirect effect on creative performance through diversity climate. The contributions of this study to three major bodies of literature, as well as the implications of the results for research and practice, are discussed. 


\section{Dedication}

This dissertation is dedicated to Bonnie Smith, my mother, who instilled in me a love of learning and a desire to attain the highest level of education. This achievement is a result of regular weekend trips to the library, numerous book orders in elementary school, and strong encouragement to read as a child and young adult. She gave me the greatest gift in the world - a hunger to learn from everyone and everything around me, especially books. Thank you, Mom! I love you.

I dedicate the completion of my doctoral program to Gene Smith - the walk across the stage is for you, Gene! I hope it makes you smile from your place in the universe. 


\section{Acknowledgements}

I would like to thank my adviser, Dr. Keith James, for making it possible for me to attend graduate school. I am grateful for the opportunity to work with this data set, which combines three of my greatest research and life interests: diversity, leadership, and creativity. While the road has been long and bumpy, it has been a privilege to study these topics in an in-depth manner. Dr. James made this achievement possible and provided helpful and constructive feedback throughout the doctoral program.

Throughout the six years of this program, my parents, Bonnie Smith, David Taylor, and Judy Taylor, have consistently offered their unconditional love, support, and understanding, even when I could not give much in return. I am incredibly grateful for all the ways they have supported me over the past six years.

Adam Murry has my undying gratitude for the hours we spent thinking about and talking through the statistical analysis and interpretation of this study. For six years, Adam has been a stalwart support in my academic pursuits and a true friend who has supported me in both big and small ways.

Finally, I would like to thank my close friends for providing a listening ear and a voice of reason during the times when motivation was difficult to muster. Specifically, I express gratitude to Cailin Daly, Rekha Rao, Brooke Dayton, Kealani Balfour, Sarah Van Dyck, Roxana Gutierrez, and Kathleen Celmins. I could not have completed this study or this program without the support of those named here and many others. It truly does take a community, and I am very grateful to have such a supportive and loving one. 


\section{Table of Contents}

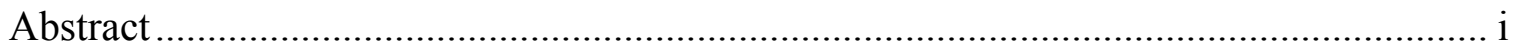

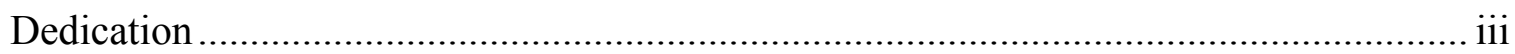

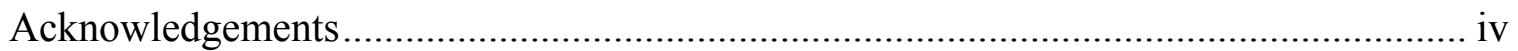

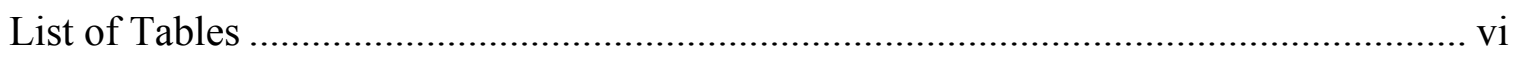

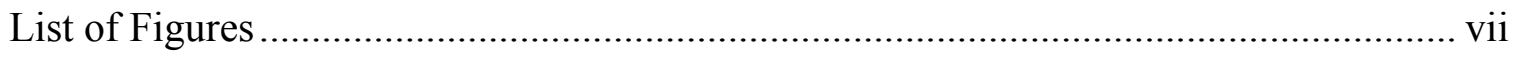

Chapter 1: Theoretical Design and Overview............................................................

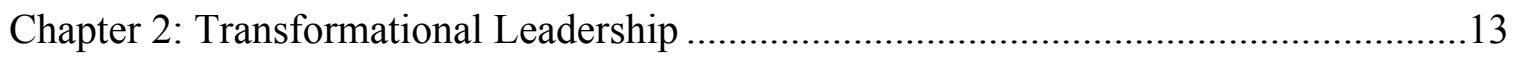

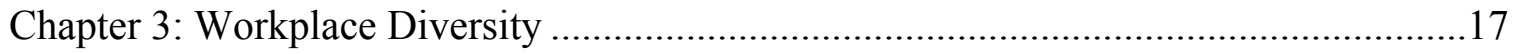

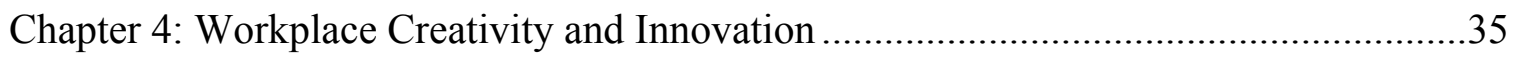

Chapter 5: Prior Research and Hypotheses...........................................................43

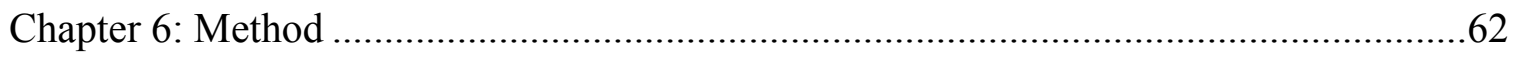

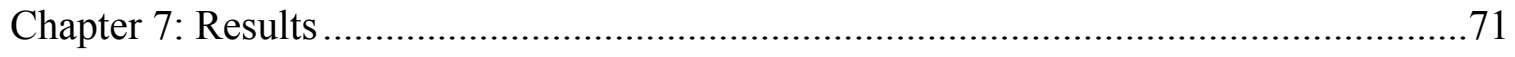

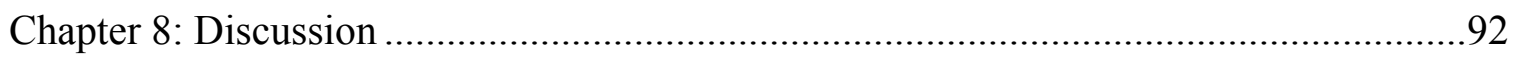

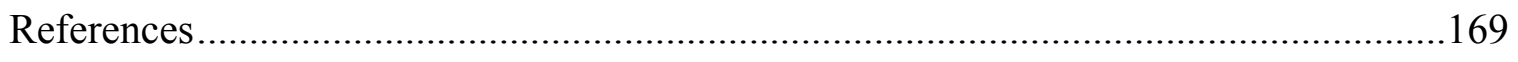

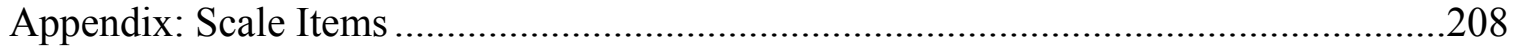




\section{List of Tables}

Table 1. Typology of Work Team Diversity..............................................................138

Table 2. Overview of Measures by Source and Data Collection Timing.

Table 3. Results of Single-level Confirmatory Factor Analyses for Main and Control

Variables

Table 4. Results of Multilevel Confirmatory Factor Analyses for Main and Control

Variables.

Table 5. CFA Results of Comparative Models for Transformational Leadership

Table 6. CFA Results of Comparative Models for the Workplace Diversity Inventory.143

Table 7. Means and Standard Deviations of Demographic Variables.

Table 8. Means, Standard Deviations, Reliability Estimates, and Correlations for Main and Control Variables.

Table 9. Level of Nesting in Focal Variables by Team...............................................146

Table 10. Level of Nesting in Focal Variables by Organization.....................................147

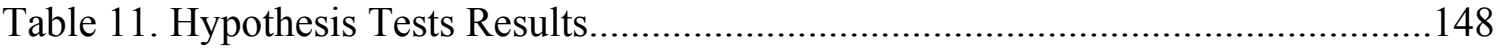

Table 12. Model Fit Indices for ECP Model and SCP Models.

Table 13. ECP Model: Single-level Path Analysis Results for Taxonomy of Workplace

Diversity Variables

Table 14. ECP Model: Single-level Path Analysis Results for Self-rated Creative Performance

Table 15. Pseudo R-Square Values for Multi-level Models.

Table 16. ECP Model: Multi-level Path Analysis Results for Taxonomy of Workplace Diversity Variables

Table 17. ECP Model: Multi-level Path Analysis Results for Employee Self-rated Creative Performance

Table 18. SCP Model: Multi-level Path Analysis Results for Taxonomy of Workplace

Diversity Variables 155

Table 19. SCP Model: Multi-level Path Analysis Results for Supervisor-rated Creativity

Table 20. Results of Indirect Effects Testing.

Table 21. Exploratory SCP Model: Multi-level Path Analysis Results for Taxonomy of Workplace Diversity Variables.

Table 22. Exploratory SCP Model: Multi-level Path Analysis Results for Self-rated Creativity.....

Table 23. Exploratory SCP Model: Multi-level Path Analysis Results for Supervisorrated Creativity 


\section{List of Figures}

Figure 1. Hypothesized Relationships between Leadership, Diversity, and Creativity...161

Figure 2. Expanded Componential Theory of Creativity .162

Figure 3. ECP Model: Single-level Path Analysis Results...........................................163

Figure 4. ECP Model: Multi-level Path Analysis Results............................................164

Figure 5. SCP Model: Multi-level Path Analysis Results............................................165

Figure 6. Exploratory Multilevel Path Analysis Results with ECP Predicting SCP......166 Figure 7. ECP Model: Interaction of Transformational Leadership and Diversity Climate in Predicting Organizational Justice. 167

Figure 8. Multilevel ECP Model: Interaction of Transformational Leadership and

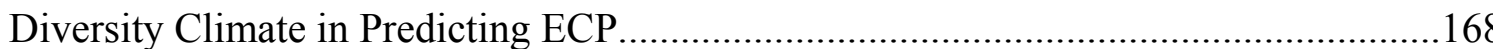




\section{Chapter 1: Theoretical Design and Overview}

Modern organizations continually strive for ways to enhance employee creativity and innovation. In today's globalized economy, creativity and innovation help organizations differentiate themselves from other highly productive competitors. Creativity fuels the processes through which firms create new products, improve services, and reduce costs (Jackson \& Joshi, 2011). To foster innovation, organizations seek employees who think critically, question assumptions, and take part in creative processes, and this fact is particularly true for high-tech firms. Employees who demonstrate creativity at work help the organization develop novel and useful product ideas and effective solutions to the constant changes and increasingly complex situations encountered in today's workplaces. These ideas and solutions often determine the difference between thriving in the face of consistent change or failing due to a lack of responsiveness and ability to adapt. Thus, understanding the conditions that facilitate creativity and innovation is essential to an organization's long-term survival. In order to develop this understanding, research is necessary to provide insight into how to teach, apply, and manage human creativity (Amabile, 1996; Csikszentmihalyi, 1999).

\section{Leadership, Diversity, and Creativity}

In this study, transformational leadership, diversity, and creativity are studied simultaneously in an attempt to better understand how to enhance individual creativity and leverage the potential benefits of diversity at work. The increasingly diverse global workforce may prove to be an opportunity for meeting the challenge of understanding 
and better predicting creativity and innovation in the workplace, since increased creativity is the most commonly cited benefit of a more diverse and inclusive workplace (Jackson \& Joshi, 2011). There is a general consensus among U.S. employers that effectively managing diversity is mandatory for organizations that seek to leverage all of the talent available in a diverse workforce (Jackson \& Joshi, 2011). Furthermore, employers in other countries are increasingly recognizing diversity as a potential organizational asset (Lester, 2006; Mangaliso \& Nkomo, 2001; Nishii \& Özbilgin, 2007). However, research shows that workplace diversity has the potential to either enhance or diminish performance (Hülsheger, Anderson, \& Salgado, 2009; Jackson \& Joshi, 2011; van Knippenberg \& Schippers, 2007). Leadership has been found to be an important predictor of whether the potential advantages of diversity will be realized and leveraged within an organization (Wieland, 2004). Since one of the beneficial outcomes of diversity is increased employee creativity, leadership that can effectively manage diversity and promote inclusion is critically important as an antecedent of workplace creativity (Ely \& Roberts, 2006; Mor Barak, 2011). This study predicts that there will be indirect effects of the leadership behaviors demonstrated by supervisors on employee levels of creative performance through employee perceptions of three different aspects of workplace diversity.

Scholars agree that effective organizational leadership is vital to leverage the benefits of workplace diversity and to prevent the potential detriments, but there is still much that needs to be understood regarding which leader behaviors help to foster the competitive advantages of diversity (Cox, 1993, 2001; Dahm, Willems, Ivancevich, \& 
Graves, 2009; Stockdale \& Cao, 2004). Organizational leaders must proceed with caution due to the mounting evidence demonstrating that poorly managed workplace diversity can result in deleterious effects on individual, team, and organizational creative and task performance (Ely \& Roberts, 2006; Guzzo \& Dickson, 1996; Milliken \& Martins, 1996; van Knippenberg \& Schippers, 2007). Therefore, the way in which diversity is managed (i.e., leadership) plays a vital role in the relationship between diversity and creativity. In light of the pervasiveness of this assertion, it is surprising that empirical research examining these three constructs of leadership, diversity, and creativity in tandem is largely absent. One of the major contributions of this study is to fill this gap in the literature: that is, to examine the combined impact of leadership and diversity on employee creativity. Due to the real-world and conceptual complexity and multidimensionality of diversity (Mor Barak, 2011; Taylor, James, \& Murry, 2012), as well as its impact on critical organizational outcomes, one of the foremost challenges for organizational scholars is to understand the dynamics of diversity well enough to be able to offer evidence-based, practical advice regarding specific actions that leaders and supervisors can take to manage diversity effectively (Jackson \& Joshi, 2011).

\section{Leadership and Creativity}

Scholars have found that both individual and workplace variables can enhance employee creativity and innovation (Anderson, De Dreu, \& Nijstad, 2004; Zhou \& Shalley, 2011). James and Taylor (2010) are among those to put forth models describing how individual differences interact with workplace situational factors to influence motivation for creativity, noting leadership as one key contextual factor (see also Avolio 
\& Bass, 2004; Bass \& Riggio, 2006). Leadership can help determine whether or not employees demonstrate creative behavior, and whether the creative behavior they exhibit is positive or negative from the perspective of the employer (Cropley, Cropley, Kaufmann, \& Runco, 2010; James \& Taylor, 2010; McLaren, 1993; Tierney \& Farmer, 2004).

After over a hundred years of research on leadership, transformational leadership has emerged as the forerunner among many competing theories (Barling et al., 2011). The research suggests that transformational leadership may be the most beneficial in terms of managing increasingly diverse workforces (Bass, 1997; Den Hartog et al., 1999; DeRue, Nahgang, \& Wellman, 2011; Wieland, 2004). There are four dimensions of transformational leadership: 1) charisma/idealized influence, 2) inspirational motivation, 3) intellectual stimulation, and 4) individualized consideration (Judge \& Piccolo, 2004; Barling et al., 2011). A more complete description of each dimension is provided in the next chapter. However, as an overview, they correspond to the following behaviors: 1) employs charisma and acts with integrity to instill pride and increase employee optimism and motivation, 2) develops and articulates a clear vision and plan for the future that motivates peers and direct reports, 3) intellectually stimulates direct reports by encouraging them to be creative, and 4) provides specific and customized attention to individual differences among people (Judge \& Piccolo, 2004).

\section{Research Questions and Proposed Model}

The purpose of this study is three-fold. First, it examines organizational diversity climate as a mediator of the relationship between transformational leadership and 
employee creative performance. Second, it asks whether transformational leadership and diversity climate work in a multiplicative fashion (i.e., interact) to promote employee creativity, such that higher levels of transformational leadership behaviors plus relatively positive perceptions of diversity climate lead to enhanced creative performance. Third, this study examines transformational leadership and organizational diversity climate to see if they help facilitate employees' sense of both organizational justice and identification with the organization, such that justice and identity mediate effects on creativity.

To fulfill the above purposes, a study was developed based on three bodies of literature: transformational leadership, organizational diversity, and creativity. The vast majority of research on workplace diversity and employee creativity has been conducted among U.S. workers. Thus, the generalizability of this research may be limited. To help increase the representation of non-Western participants in the literature, and in light of the emergence of China as a major player in the global economy, this study was conducted among a sample of Chinese participants.

In this study, transformational leadership and diversity climate are predicted to interact to significantly influence perceived organizational identity, organizational justice, and individual creative performance. It is also proposed that organizational identity and organizational justice are partial mediators of the relationship between the leadershipdiversity climate interaction and creativity. Both constructs—organizational justice and organizational identity—are examined in the context of a workplace diversity theoretical framework, developed by Taylor and colleagues (2012). That is, the constructs are 
theoretically similar to the general constructs commonly examined in the literature, but they are theorized to be examined in the specific context of workplace diversity. Figure 1 depicts a moderated mediation model that illustrates the hypothesized relationships to be tested within the study. The model was analyzed using multi-level path analyses, since the constructs in the model are predicted to correlate with each other. This analysis allows for testing of the unique effects of each predictor, with the correlations among the variables taken into account.

The data for this study was collected in three waves, and the focal variables include employee ratings of supervisor transformational leadership; employee perceptions (i.e., self-report) of diversity climate, organizational justice and organizational identity; and supervisor and self-rated employee creative performance. In addition to supervisor ratings, which is important to organizations in terms of both employee outcomes and supervisor evaluations, individual creative performance was measured by self-ratings because scholars have argued that creativity is a process (Drazin, Glynn, \& Kazanjian, 1999) and focal individuals are likely to be the first ones to be aware of their own engagement in it, whereas co-workers and supervisors are likely to notice only once creative outcomes have been achieved (Zhou \& Shalley, 2011). Several recent studies have asked employees to report their own creativity at work (Carmeli \& Schaubroeck, 2007; Kark \& Carmeli, 2008; Shalley, Gilson, \& Blum, 2009; Zhou, Shin, \& Cannella, 2008). This study followed the relatively recent development by using an employee self-rated measure of individual creative performance; however, the more conventional supervisor-rated measure was also used. It was predicted that supervisor- 
and self-rated creativity would be related to each other, and this study also tested that relationship.

While it may seem that China is ethnically homogenous, there are 56 ethnic groups in China. The Han ethnic group (or "Han nationality") comprises approximately $91 \%$ of the population, which numbers more than 1,300,000,000 (Ohio State, 2013). Thus, in order to obtain the potential benefits of diversity within Chinese organizational contexts, it is important to foster a diverse workforce and ensure employees of all ethnicities perceive they are included in the organization and in their work teams. That is, it's important to build a positive diversity climate to foster the creativity that will enable the employees to meet the needs of customers in the fast-paced and quickly changing global marketplace. In this study, the measures of diversity are focused on internal dynamics within the organization. At the same time, fostering diversity and building inclusion is critical for Chinese organizations, so they can understand and respond to the needs of external, global customers. Building a positive diversity climate is not only important for critical organizational outcomes (e.g., retention, job satisfaction, organizational commitment), it is essential for the development of products that appeal to a diverse, global clientele, which requires a certain amount of innovation (i.e., creative performance).

Motivation is an important concept in the proposed model, with three of the five focal variables being conceptually similar to motivation, either explicitly (e.g., the inspirational motivation dimension of transformational leadership) or implicitly. Regarding the implicit conceptual similarity, organizational justice is often described as a 
theory of motivation in the literature. In addition, according to the Componential Theory of Creativity (Amabile, 1996), a key antecedent to, or "component" of, creative performance is intrinsic motivation to be creative. Thus, organizational justice serves as an implicit measure of motivation within the model, which asserts that it will be predicted by transformational leadership (especially its inspirational motivation dimension), and will lead to increased employee creativity.

Motivation commonly has implications at the meso, or team, level. While it is important to study transformational leadership, workplace diversity, and employee creativity at this level of analysis (i.e., team), this study focuses on these predictors and outcomes at the individual level because the model is new and complex, even when only examining the constructs at the individual level. Future research should explore these relationships further as they relate to team-level variables.

\section{Contributions of the Research}

This study is unique in proposing and testing a model in which two distinct contextual factors - transformational leadership and diversity climate-foster employee creativity through enhanced organizational justice and organizational identity. While research has been conducted on the relationships between pairs of these constructs, the unique contribution of this study lies in the examination of these constructs at the same time, using multi-level path analyses to account for the correlations among them. No other studies have examined the interaction of transformational leadership and diversity climate to impact creativity. It follows that there is no prior research on the mediators of this proposed interactive effect; this study proposes that organizational justice and 
organizational identity are mediators of the relationship between creative performance and the interaction of transformational leadership and diversity climate.

The research at hand stands to make three substantive contributions to the organizational literature. First, this research responds to the urging of scholars to use a well-elaborated construct that has been extensively tested in various research fields and in many cultures (i.e., transformational leadership; Barling et al., 2011; Bass \& Avolio, 1990) to examine the relationship between diversity and creativity. As discussed in a subsequent chapter, multiple measures of diversity are employed in this study to reflect the multidimensional nature of the construct and to provide a more comprehensive examination of it. By examining the ways in which transformational leadership and multiple aspects of workplace diversity (i.e., diversity climate, organizational justice, and identity) interact and/or relate to impact individual creative performance, more will be known about the leader behaviors that are effective in diverse workplaces be bring about increased creativity, as well as the mechanisms through which leadership impacts diversity and, ultimately, creative performance. Specifically, this study was designed to examine the interaction between transformational leadership and diversity climate as well as possible mediators of the relationship between leadership and creative performance. This study provides insight to scholars and practitioners to better understand the multiple and complex relationships of leadership, diversity and creativity.

The second contribution of this study is that it is conducted outside of the U.S. and in an Asian country, namely, China. The majority of studies on diversity and creativity have been conducted within Western countries (Jackson \& Joshi, 2011; Zhou \& 
Shalley, 2011), so the use of a Chinese sample of employees will enrich these two bodies of literature. It also responds to calls in the literature for exploration of leadership, diversity, and creativity processes to be conducted outside of the U.S., especially in emerging Asian economies (Drazin \& Schoonhoven, 1996; Jackson \& Joshi, 2011). In addition, while transformational leadership has recently been studied with relative frequency in China (Aryee, Walumbwa, Zhou, \& Hartnell, 2012; Si \& Wei, 2012; Zhu, Newman, Miao, \& Hooke, 2013), a meta-analysis examining articles published between 1985 and 2006 reveals that the majority of research on transformational leadership has been conducted in Western countries (Leong and Fischer, 2011). The meta-analysis included 40 published articles and 54 independent samples from 18 nations that used the Multifactor Leadership Questionnaire to measure transformational leadership. It lists 10 studies on transformational leadership conducted in Eastern countries (including one in China, one in India, two in South Korea, two in Taiwan, and four in Singapore) while 16 studies were conducted in the U.S. alone, and 32 in all Western countries combined. Thus, using a Chinese sample to study transformational leadership is important in itself. In light of China's expanding role in the global economy, multinational companies are increasingly moving "knowledge-creating" jobs to Chinese cities (Scullion \& Collings, 2011). Moreover, Chinese companies have tended to rely on technologies and products developed elsewhere, but these companies have a vested interest in increasing domestic creativity and innovation. Thus, it is important to conduct research on leadership, diversity, and creativity in Chinese workplaces. This study provides an investigation of these variables among Chinese employees working in their native 
country, and it is unique in the way in which transformational leadership is modeled and tested in combination with workplace diversity and employee creativity.

The final potential contribution of the current study is that it may provide empirical evidence to support the theoretical predictions in the literature that organizations' social context interact with leadership to influence employee creativity. Conceptual models (e.g., Amabile, 1988, James \& Taylor, 2010) have noted the relevance of social context for employee creativity. The application of transformational leadership and workplace diversity theories to creativity, and the examination of the interaction and relationships among these constructs, may shed light on the mechanisms by which the context of an employee's working environment encourages (or discourages) creativity. The findings of this study complement and augment existing interactionist models, which theorize employee creativity as a complex product of personal attributes, behaviors, and situations (e.g., Amabile, 1988; Woodman et al., 1993; James \& Taylor, 2010).

The findings of this study may help guide organizational leaders leverage diversity and, in turn, promote creativity. Much of the organizational literature on diversity management is predominantly focused on organizational change and training interventions; both of which require a large investment of resources. If leadership is found to significantly interact with diversity climate to influence creativity, organizational interventions can focus on leadership selection and performance evaluation processes, leadership development objectives, and promotion criteria for leaders, which often require fewer organizational resources. Organizations may seek to 
hire more transformational leaders, focus supervisor training on developing the behaviors characteristic of transformational leadership (evidence has been found that the four dimensions of transformational leadership can be developed; Barling et al., 2011), or evaluate employees based on these behaviors to encourage and provide accountability for supervisors to enact them. In the following chapters, the theoretical foundations of the three main constructs of interest (leadership, diversity, and creativity) are reviewed. These chapters detail the current research findings and outline the hypotheses regarding the proposed relationships in the study. Subsequent chapters describe the sample, procedure, data analysis, results, and implications of the study. 


\section{Chapter 2: Transformational Leadership}

Western study of leadership has a long and rich history in the social sciences, particularly in Industrial/Organizational Psychology. In the past century, scholars have taken a variety of approaches to researching leadership, including trait-based theories, analysis of leader behavior, situational contingencies, and relational theories (i.e., leadermember exchange [LMX] theory). However, in the past thirty years, one conceptualization of leadership — transformational leadership theory-has emerged as the most dominant and widely studied (Barling, Christie, \& Hoption, 2011; Bass \& Riggio, 2006; Judge \& Bono, 2000; Judge \& Piccolo, 2004). One reason for the prevalence of research on transformational leadership is that it has been found to be highly effective in terms of overall employee performance, task performance, and affective/relational measures (e.g., LMX, follower satisfaction; DeRue et al., 2011).

\section{Theoretical Foundation}

The modern theoretical foundation of transformational leadership is generally considered to have been established by two influential books. In the first, simply titled "Leadership", political scientist James McGregor Burns (1978) coined the term "transformational leader" and differentiated transformational leadership from other forms in the context of political leadership. Burns asserted that transformational leaders engage others in a two-way process, "in such a way that leaders and followers raise one another to higher levels of motivation and morality," which ultimately "raises the level of human conduct and ethical aspiration of both leader and led, and thus has a transforming effect on both" (1978, p. 20). In the second book, Bass (1985) used Burns' conceptualization 
and extended the focus of the construct to the organizational context. In addition, Bass elaborated on the behaviors that set transformational leaders apart from others. Although there have been a number of iterations of the theory, the most recent (and best supported) version includes four dimensions of transformational leadership, which are charisma/ idealized influence, inspirational motivation, intellectual stimulation, and individualized consideration (Judge \& Piccolo, 2004; Barling et al., 2011).

Charisma/idealized influence. This dimension refers to leader behaviors that provide a model for ethical behavior and inspire employees to act in the best interest of the organization, instead of what may be most efficient and convenient. When displaying charisma, transformational leaders act in ways that build respect and trust, instill pride, and increase optimism (Bass, 1985). Idealized influence is the degree to which a leader demonstrates admirable behaviors that cause followers to identify with him/her (Judge \& Piccolo, 2004). A defining characteristic of idealized influence is acting with integrity (Barling et al., 2011). Common behaviors of this dimension have been noted in the literature to include displaying conviction, taking a stand, appealing to followers on an emotional level, speaking with a captivating tone of voice, making eye contact as appropriate with the listener, having animated facial expressions, and communicating in a powerful, confident, and dynamic way (Judge \& Piccolo, 2004; Barling et al., 2011).

Inspirational motivation. This dimension of transformational leadership refers to a leader's ability to develop and communicate a compelling vision for the future. By doing so and setting high but realistic standards to achieve the vision, transformational leaders instill in others the belief that they can achieve more than previously thought, go 
beyond expectations, and overcome current and future hurdles. Inspirational motivation can be transmitted through interpersonal interactions, e.g., telling stories and using symbols (Barling et al., 2011; Bass, 1985).

Intellectual stimulation. In contrast to prevailing notions of good leaders as those who can answer all questions posed by employees, this dimension describes leaders who obtain input from others when problems arise and challenge and encourage employees to think critically and be creative (i.e., to "think outside the box"). When supervisors employ this aspect of transformational leadership, they also encourage employees to question commonly held assumptions, reframe problems, take appropriate risks, and approach challenges in innovative ways (Barling et al., 2011). In addition, a defining behavior of intellectual stimulation is providing a steady flow of new and challenging ideas to employees, which in turn arouses their imagination and empowers them to feel more confident and self-efficacious in work-related matters (Judge \& Piccolo, 2004).

Individualized consideration. The final dimension of transformational leadership refers to a leader's willingness and ability to pay close attention to an individual's specific development needs and to act as a mentor and/or coach by providing continuous feedback and linking individual passions and aspirations to the organizational mission (Bass, 1985). It includes displaying caring, compassion, and empathy to employees, which positively influences employee well-being by providing instrumental and emotional support. Supervisory support helps employees develop their potential and 
skills, as well as healthy working relationships with their supervisors and co-workers

(Barling et al., 2011). 


\section{Chapter 3: Workplace Diversity}

In the U.S. and internationally, workplaces are becoming more diverse, and effective workplace diversity management is increasingly critical to organizational success (Cox, 2001; Mor Barak, 2011; Triandis, 2003). Today's workforce is more heterogeneous than that of previous generations along many social categories (e.g., age, gender, ethnicity, national origin), and research suggests that this trend will only accelerate in the future (Judy \& D'Amico, 1997; Stockdale \& Cao, 2004). Due to the rapid rate of globalization, working with colleagues from different nations is also much more common (Haq, 2004; Scullion \& Collings, 2011). The reality of today's workforce creates a vital need for employees to appreciate and value diversity in order to work more effectively with people from different social groups and various cultural backgrounds. Doing so may provide organizations with a competitive advantage in the marketplace, and research has supported this assertion; it has been found that certain types of diversity result in enhanced creativity, better decision-making, and ultimately, increased profitability (Jackson \& Joshi, 2011).

\section{Defining Workplace Diversity}

The term diversity is used often and in many different ways. Mor Barak (2011) provided a list of thirty definitions of diversity developed by scholars from 1991 to 2010 . Furthermore, numerous authors have discussed workplace diversity in relation to phenomena at the individual, team, and organizational levels, often using different names for the construct (Hays-Thomas, 2004; Stockdale \& Cao, 2004). Multiple potential levels 
and various apparent elements of a construct imply the need for a multidimensional taxonomy, rather than a simple, single conceptual definition. Thus, the need for a taxonomy of workplace diversity has become apparent in the extant literature. Taxonomy is the science or technique of classifying the dimensions of a construct or concept into ordered categories, and many industrial/organizational psychology scholars have developed taxonomies of psychological constructs, such as managerial goals (Bateman, O’Neill, \& Kenworthy-U'Ren, 2002) and organizational justice theories (Greenberg, 1987). While taxonomies are more commonly used in the natural sciences (Fleishman, Quaintance, \& Broedling, 1984), they are helpful in the behavioral sciences because they enable improved understanding, description, and categorization, which in turn enable improved prediction and control (Bateman et al., 2002).

With this in mind, Taylor and colleagues (2012) developed a taxonomy of the psychological dynamics and patterns that are present in diverse U.S.-based and international work settings to provide a more complete description and precise definitions of the key dimensions of workplace diversity (Taylor, James, \& Murry, 2012). An instrument was also developed and tested for reliability and validity, with promising results, called the Workplace Diversity Inventory (WDI; Taylor \& James, in progress). To establish evidence for construct validity of the taxonomy, i.e., to precisely answer the question of what is workplace diversity, it is important to develop the nomological network of the construct. That is, due to the large number of definitions and ways of using the term, as well as the multiple scales that have been created to measure workplace diversity, distinguishing between what the construct is and what it is not is essential. 
Establishing a nomological network of workplace diversity entails the following: 1)

identifying the dimensions of diversity at work, 2) identifying antecedents (i.e., individual or situational factors that lead to a person's perception of a particular dimension of diversity), 3) identifying the similarities and differences between workplace diversity and other similar constructs (i.e., correlates), and 4) identifying outcomes of the construct, which concerns criterion-related validity. While development of the nomological network of workplace diversity is in its nascent stage, the taxonomy developed by Taylor and colleagues has helped to clarify the multidimensional nature of the construct. This study and subsequent research will build on this foundation.

While the taxonomy of workplace diversity, and the instrument developed to measure it, provides an operational definition to move research on this topic forward, the following definition of workplace diversity is offered in the context of this study. It was developed by Mor Barak (2011) and recently modified (K. James, personal communication, September 26, 2012):

Workforce diversity refers to the division of the workforce into distinct categories that (a) have a perceived commonality within a given cultural or national context and that either: (b) increase potentially harmful or decrease beneficial employment outcomes for individuals and groups for reasons other than jobrelated skills and qualifications or/and (c) negatively impact inter-individual, intra-team, inter-group, or organizational achievement of performance potential.

The necessity and vitality of this definition lies in the fact that it focuses on diversity within a global context by providing a way to include categories (e.g., regional differences, profession, HIV status) that may be relevant in some cultures or contexts but not in others. From the taxonomic perspective, context then becomes another way to describe relevant aspects of diversity. This definition also emphasizes the importance of 
the consequences of social categorization in terms of its potential to affect important workplace outcomes, which addresses the limitation of broad definitions of diversity that include inconsequential characteristics.

The dimensions of the workplace diversity taxonomy include the following: identity, values, schemas, communication, organizational justice, and diversity climate (Taylor et al., 2012). For the purposes of this study, three of the six dimensions are examined, which include diversity climate, organizational justice and organizational identity, due to the theorized relationships among transformational leadership, creativity, and these dimensions. Empirical research has also pointed toward the likelihood of the theorized relationships, and these findings will be outlined in a subsequent section. Descriptions of the three dimensions are provided below.

Diversity climate. Many organizations have implemented diversity initiatives for a number of different reasons, and the success of these efforts often depends on the broader context of the organization, i.e., diversity climate (Kossek \& Zonia, 1993; Rynes \& Rosen, 1995). Diversity climate has been defined in the literature as employees' shared perceptions (at the organizational or team level) of the degree to which the organization demonstrates that it values diversity within the workplace (McKay, Avery, \& Morris, 2008; Mor Barak, Cherin, \& Berkman, 1998; Rotundo, Nguyen, \& Sackett, 2001). Diversity climates are essentially internalized beliefs about past team or organizational diversity practices, and current team or organizational diversity attitudes, norms, and policies. The main characteristics of a positive diversity climate include public support from top management, supportive policies, and a high organizational priority on diversity 
(Rynes \& Rosen, 1995). In the workplace diversity taxonomy, then, diversity climate is defined as the extent to which employees perceive that an organization's policies, practices, and procedures emphasize fostering and maintaining diversity as well as the goal of deriving benefits from diversity (Taylor et al., 2012). Note the link of the latter part of the definition to the idea of deriving globally-competitive creativity from employees. That potential link between diversity climate and creativity is elaborated in Chapter Four.

Organizational justice. While scholars have noted the conceptual similarities of organizational justice and diversity climate, the two concepts have been distinguished theoretically and empirically (McKay, Avery, \& Morris, 2008; McKay et al., 2007). Organizational justice concerns only the fairness component of diversity climate, while the latter construct encompasses the components of organizational structure and social integration.

Greenberg (1987) defined organizational justice as an individual's perception of fairness in organizations, along with the associated behavioral, cognitive and emotional reactions. Organizational justice is generally conceptualized as having the following four dimensions: distributive, procedural, interpersonal, and informational (Colquitt et al., 2001). Often, interpersonal and informational are collapsed into one dimension called interactional justice, referring to the interpersonal treatment of others when implementing policies (Colquitt et al., 2001). Distributive justice refers to the fair allocation of resources and rewards, while procedural justice refers to the general fairness of how organizational policies and procedures are implemented. Previous research has 
found evidence to suggest that workers' perceptions of organizational fairness are central to diversity management (Ely \& Thomas, 2001; Roberson \& Colquitt, 2005). Roberson and Stevens (2006) identified organizational justice as a consideration in employees' attributions of diversity-related incidents. Within the workplace diversity taxonomy, organizational justice is defined as the extent to which employees perceive fairness of the distribution of resources, procedures, and interactions within a diverse organization (Taylor et al., 2012).

Most research on organizational justice has been conducted in North America and Europe (Li \& Cropanzano, 2009). Given this construct's centrality in Western organizational theories and research, more work is needed on the effects of it in Asia, generally, and in China particularly. This study specifically addresses this need.

Organizational justice has also been linked to creativity in some research (e.g. James, in progress; Schepers \& van den Berg, 2007). James (in press) found when employees focused their attention on organizational injustice, employees displayed less creative behavior (i.e., compared with a neutral focus of attention, employees generated fewer creative ideas and the average novelty of ideas decreased). Schepers and van den Berg (2007) found that knowledge sharing mediated the relationship of cooperative-team perceptions and procedural justice (i.e., one form of organizational justice) with creativity. As with diversity climate, more thorough theoretical rationales and empirical reviews of the justice and creativity relationship are provided in Chapter Four.

Because organizational justice is theorized in this study to be examined in the context of workplace diversity, the items used to measure it are specific to this context. It 
follows that commonly used general measures of organizational justice would not precisely assess the construct used in this study, so the instrument used to assess organizational justice in this study was chosen accordingly.

Organizational Identity. This construct is defined as the extent to which one perceives belongingness and inclusion in work teams or the workplace overall (Taylor et al., 2012), "where the individual defines him or herself in terms of the organization(s) in which he or she is a member" (Mael \& Ashforth, 1992, p.104). In this context, organizational identity encompasses the social or informal aspects of an employee's perception that he or she is an integral part of the organization, department, or work group.

Perceived identity shapes peoples' in-group and out-group perceptions, emotions, and behaviors (Hogg \& Terry, 2000; Turner, 1981), and in the context of the workplace, both intra-group and inter-group feelings and relations are affected (Hogg \& Terry, 2000; Messick \& Mackie, 1989). While in-group perceptions are important for positive selfworth, distinctions made between in-groups and out-groups at work can bring about exclusion, discrimination and prejudice based on one's perceived social identity (James et al., 1994). Additionally, individuals' own organizational identity influences their thinking and behavior about other aspects of work (Mor Barak, 2011). Such influences include organizational identity impacts on creative thinking and behavior (Cohen-Meitar, Carmeli, \& Waldman, 2009). Cohen-Meiter and colleagues (2009) found that organizational identification is positively associated with supervisor ratings of employee 
creativity. A theoretical justification and a review of the empirical evidence for the organizational-identity to creativity relationship are elaborated in Chapter Four.

Similar to organizational justice, organizational identity is theorized to be examined in the context of workplace diversity, so the items used to measure it are specific to this context. Thus, commonly used general measures of organizational identity would not adequately assess it, and the instrument to assess this construct was chosen accordingly.

\section{Types of Diversity}

In addition to the different levels (i.e., individual, team, organization) and dimensions (e.g., values, identity, organizational justice) of diversity described in the previous section, there are also different types of diversity. It is useful to differentiate among various types of diversity because the research seems to indicate that important workplace outcomes vary by the type of diversity considered (Jackson \& Joshi, 2011). Identifying the particular types of diversity used in studies on workplace diversity and its consequences may be useful for interpreting past and future research. Jackson and Joshi (2011) modified a typology developed by West, Tjosvold, and Smith (2003; see Table 1).

The first column of the typology is relations-oriented diversity, which includes attributes (e.g., age, gender, ethnicity) that are instrumental in shaping interpersonal relationships but which typically have no apparent direct implications for task performance (Jackson \& Joshi, 2011). However, there is evidence that some types of relations-oriented diversity are related to psychological differences in attitudes and values (Jackson \& Joshi, 2011). The second column is task-oriented diversity, which 
includes attributes that are potentially relevant to performance (e.g., organizational tenure, formal credentials, cognitive abilities). Evidence has also been found to suggest that some types of task-oriented diversity are related to psychological differences in attitudes and values. For example, in the case of organizational tenure, it has been found that executives tend to become more committed to the status quo the longer they stay in the same organization (Finkelstein \& Hambrick, 1990).

The first row of the typology is readily detected diversity, which includes surfacelevel differences that are generally easy to recognize and identify, such as age, gender, and nationality. The second row is underlying diversity, which include differences that only become known through interaction, such as attitudes and skills. Of course, there are exceptions to these categorizations, especially in the complex society in which we live. For example, in the case of the readily detected attributes, many of those listed are not always immediately recognizable, such as one's ethnicity, religion, or gender. However, they are thought to be more readily identified than the attributes categorized as underlying diversity. The main point of distinguishing among the various types of diversity is the notion — thus far supported by the research — that underlying diversity has greater potential to lead to positive benefits (i.e., for the purposes of the current study, employee creativity) than other, more readily detected forms of diversity (Hülsheger, Anderson, \& Salgado, 2009; Jackson \& Joshi, 2011).

Over the past ten years, comprehensive reviews of the literature and meta-analytic studies have revealed that the findings on work team diversity have begun to converge into some discernible patterns, at least on the more commonly studied dimensions of 
demographic diversity (for comprehensive reviews, see Jackson, Joshi, \& Erhardt, 2003; Jackson, May, \& Whitney, 1995; Joshi \& Roh, 2007; Milliken \& Martins, 1996; Webber \& Donahue, 2001; van Knippenberg \& Schippers, 2007; Williams \& O’Reilly, 1998). Jackson and Joshi (2011) summarized these convergent findings into the two categories described above: relations-oriented diversity and task-oriented diversity. First, they conclude that relations-oriented diversity in work teams is often (but not always) of little consequence, at least for outcomes that have been examined to date. A recent metaanalysis of 69 effect sizes between relations-oriented diversity and team performance found the average effect size to be -.03 (Joshi \& Roh, 2009). However, occupational demography seems to be a moderator of the relationship between relations-oriented diversity and team performance (Joshi \& Roh, 2009). This moderating condition suggests that the outcomes of workplace diversity depend in part on context (Jackson \& Joshi, 2011). In the case of the current study, variations in levels of transformational leadership and in perceptions of diversity climate are the contextual factors of interest. Given that the study was conducted with organizations in China with all-Chinese employees and leaders, racial or ethnic diversity was not relevant. Other types of diversity (e.g., age, organizational tenure) were pertinent, however, as detailed in the Method chapter.

Second, the research findings on task-oriented diversity clearly suggest that diversity on characteristics such as functional background, education, and job or organizational tenure is often likely to enhance team performance (Jackson \& Joshi, 2011). In Jackson and Roh's (2009) meta-analysis, functional diversity had the strongest 
positive relationship with team performance (.13), followed by tenure diversity (.03) and educational diversity (-.02).

The implications of these findings for research are that scholars should precisely define which types and which dimensions of diversity they are examining. Because there is convincing evidence that the different types of diversity result in different outcomes, it seems imperative that diversity scholars become increasingly more specific. One goal of this study is to precisely describe the dimensions of diversity under examination and detail the differences in how they relate to each other and to the outcome of interest, i.e., individual creative performance. A practical implication of these findings is that employers should not assume that certain types of diversity would help or hurt individual, team, and organizational performance. Because the research clearly shows that diversity can have positive and negative effects, organizational leaders should examine their specific work contexts, the specific types of diversity within it, and determine which outcomes are most important. A further goal of this study is to shed more light on the specific ways in which employers can leverage diversity within teams and in the organization as a whole.

\section{Theoretical Foundations}

The model in this study was developed by considering four theories that have guided research on diversity at work, including attraction-selection-attrition (ASA), social identity theory, the information processing perspective, and the faultline perspective. The theories are explained below, along with a brief summary of how they provide the theoretical foundation for the hypothesized relationships in the model. For a 
complete review of the theoretical advances in understanding workplace diversity, see Van Knippenberg and Schippers (2007).

Attraction-selection-attrition. Attraction-selection-attrition (ASA; Schneider, 1987) is one of the most commonly used theories in diversity research, and it recognizes that diversity at work can be a "double-edged sword" (Jackson \& Joshi, 2011). That is, it predicts that diversity will have both positive and negative outcomes in work settings. ASA suggests that organizations tend to naturally move toward greater homogeneity because people tend to be attracted to similar others. At work, this can manifest in applicant attraction (or repulsion) to an organization because applicants look for coworkers who are similar to them. Organizational hiring managers also look for applicants with "good fit" to the organization, which is often not well defined and can often mean that the applicant is similar to many employees in the organization along readily detected attributes. The theory emphasizes the role of individual employee personalities, values, and interests that shape organizational life (Schneider, Goldstein, \& Smith, 1995). The ASA perspective provides an explanation for the gradually decreasing demographic diversity that has been found in organizations (Boone, va Olffen, van Witteloostuijn, \& De Brabander, 2004; Jackson \& Chung, 2008). This trend may result in deleterious effects for the organization in that while a homogenous organization may function more smoothly, it may lack the creativity and adaptability that is necessary to compete and be successful in today's global marketplace. In the short term, diversity may increase turnover, but this cost is worth paying if the benefits of diversity can be realized. Thus, the ASA perspective suggests organizational leadership must take proactive steps 
to increase and retain a diverse workforce. In this study, it is proposed that transformational leadership will sufficiently counter the forces predicted by ASA toward increased homogeneity within work settings by providing a broad, inspiring vision to unite a diverse workforce, motivating employees through charismatic behaviors to strive for and achieve common goals, and responding to individual employee needs to help all employees feel cared for and included.

Social identity theory. Social identity theory (Tajfel \& Turner, 1979, 1985; Ashforth \& Mael, 1989) rivals the ASA model as the most commonly used theory in workplace diversity research. The social identity perspective encompasses social categorization theory and social identity theory (Reynolds, Turner, \& Haslam, 2003). The main premise of this perspective is that individuals classify themselves and others based on overt (or readily-detected) demographic attributes. Specifically, individuals who are demographically similar classify themselves as members of an in-group, while those who are not similar are categorized as part of the out-group. The social identity perspective recognizes that similarity is based on the individual's perception of similarity and depends on which attributes an individual believes to be salient. Similarity is also dependent upon context; attributes that are considered to be similar in one group setting may be considered to be different in another. These two aspects (i.e., similarity being a social construct and being specific to situations) are in contrast to ASA, which assumes that some "objective" similarity is of primary importance. Individuals bring many attributes to each situation, but social identity theory asserts that only those attributes that are perceived to be or become salient shape behavior. It combines an understanding of 
individual-level processes with an appreciation for the role of social contexts (Chattopadhyay, Tluchowska, \& George, 2004), which is critical in the current study. The dynamics that manifest in a diverse organization must be understood within its particular context (Jackson \& Joshi, 2011). In relation to the current study, this theory highlights the importance of transformational leaders to prevent or mend in-group/outgroup conflict within the organization by working to build one single "in-group," i.e., the organization itself, and the teams or work groups within it, with all employees working toward the same vision and goals.

Information processing. The informational diversity-cognitive resource perspective (Cox \& Blake, 1991; Jackson, 1992; Nemeth, 1986, 1997) emphasizes the role of task-related resources, such as knowledge and skills. Unlike the prior theories, it predicts that a diverse workforce and diverse work teams will result in positive outcomes, and it focuses on task-oriented team activities, rather than affect-based relationships. The information processing perspective assumes that employees bring unique approaches and expertise to work-related activities (i.e., task-oriented diversity), which can result in improved decision-making in a variety of areas. Using the same logic, Harrison and Klein (2007) conceptualized diversity as a source of information, knowledge, and expertise. Likewise, Jackson (1992) suggested that diverse teams might search more broadly for information, develop more possible solutions to a problem, and engage in more rigorous debate before settling on a decision. Nemeth $(1986,1997)$ found that the presence of a minority dissenting opinion inspired an increased exchange of previously unshared information within teams. The information processing theory has been central to research 
on teams at the upper levels of organizations (Finkelstein \& Hambrick, 1996), and it has stimulated research on decision making and performance in lower-level work teams (Jehn \& Mannix, 2001). In the context of this study, it is suggested that transformational leaders and highly positive perceptions of diversity climate will interact in a multiplicative fashion to help employees feel included in the organization (identity) and to perceive that policies and procedures are being carried out in a fair manner (organizational justice) so that the predictions of the information processing theory will hold true. That is, employees will be more likely to fully engage in information sharing and be more motivated to perform in a creative manner.

Faultline theory. Finally, the faultline perspective is a relatively recent way to describe the dynamics of workplace diversity (Jackson \& Joshi, 2011). It suggests that work teams can be influenced by the formation of competing subgroups, so scholars should focus on understanding the structure of the work team diversity (Jackson et al., 1995). That is, to understand diversity's outcomes, it is necessary to know the configuration of team members' attributes. Faultline theory asserts that differences among team members are most likely to have significant consequences when they elicit the formation of distinct subgroups (Lau \& Murnighan, 1998). A faultline is present "when two or more relatively homogenous and distinct subgroups form in a team on the basis of multiple shared attributes" (Jackson \& Joshi, p. 659). Specifically, faultline theory suggests that the negative effects of team diversity are better understood by considering the influence of different types of diversity simultaneously, rather than considering each type separately. The existence of faultlines (based on differences in 
nationality and education major) has been found to disrupt information sharing and team performance (Jiang, Jackson, Shaw \& Chung, 2008), but can depend on situational conditions, such as the nature of team's task (Jackson \& Joshi, 2011). Because faultlines can be deleterious for employee performance, the introduction of transformational leadership behaviors is predicted to prevent the formation, or reduce the number, of faultlines in a diverse workforce, thus increasing the likelihood of positive outcomes such as creative performance.

While there are clear differences among these theories, all are premised on the assumption that the types and distribution of personal attributes among members partly determine how employees work together within a diverse workforce, and ultimately, how they perform (Jackson \& Joshi, 2011). There is a need to integrate these theories (Jackson \& Joshi, 2011), and some of this work is underway (Joshi, Liao \& Jackson, 2006), including the current study.

\section{Theoretical Predictions of the Transformational Leadership-Diversity Relationship}

Scholars have recently asserted that there is a paucity of research about the relationship between leadership and workplace diversity and have called for an examination of the leader behaviors or styles that are most effective for diverse workplaces (Jackson \& Joshi, 2011). This study attempts to address this gap by examining employee perceptions of leadership and diversity in Chinese work settings.

The model that will be tested in this study predicts that transformational leadership will lead to more positive perceptions of diversity, and thus to higher levels of individual creative performance. From a theoretical perspective, the behaviors and 
attributes that define transformational leadership are needed to overcome the natural movement of organizations toward greater homogeneity, as predicted by ASA. Social identity theory highlights the importance of transformational leadership in the context of workplace diversity to prevent exclusion or discrimination based on categorizations of employees to in-groups and out-groups. Transformational leadership includes acting as a role model and providing motivational inspiration and intellectual stimulation. Through these behaviors, a transformational leader aligns organizational and/or team values and goals with individual employee values and goals, thus building the individual's sense of optimism and efficacy (Avolio \& Bass, 2004; Bass \& Riggio, 2006). Because faultlines can be detrimental for the performance of work teams, it is proposed that transformational leadership behaviors will help prevent the formation or reduce the number of faultlines in the organization and among work groups, thus increasing the likelihood of enhanced creative performance.

Overall, it is proposed that transformational leadership will help leverage the positive outcomes of diversity by instilling pride in employees for the organization, emphasizing the greater good of the group, and acting in ways that build respect and trust (Bass, 1985). These behaviors serve as proactive measures that help develop a strong team or organizational orientation to neutralize the potentially negative effects of diversity on conflict (Mohammed \& Angell, 2004) and to avoid or decrease turnover. As the theories above suggest and the empirical findings demonstrate, workplace diversity holds the potential to be either beneficial or detrimental. It seems that effective leadership may be especially helpful in ensuring that employees, teams, and the entire organization 
achieve their potential for excellent performance and avoid the potential interpersonal problems that tend to result in higher turnover rates (Gibson \& Vermeulen, 2003) and other negative workplace outcomes. Since transformational leadership has been found to be effective in a variety of work settings (DeRue et al., 2011), it follows that transformational leadership will help to foster the potential benefits of workplace diversity, and evidence has been found to support this assertion (Kearney \& Gebert, 2009).

Scholars have increasingly called for research on diversity to specify both the types and dimensions of diversity under examination (Jackson \& Joshi, 2011; Taylor et al., 2012). Thus, in the current study, hypotheses are developed regarding the relationship of leadership and diversity based on three dimensions of diversity in particular, including organizational justice, identity, and diversity climate. 


\section{Chapter 4: Workplace Creativity and Innovation}

In the context of increasing globalization, rapid technological advancements, and the volatility of the global marketplace, organizations strive to inspire employee creativity and innovation in order to obtain and maintain a competitive edge. Creativity has been theorized to be a key contributor to performance, growth, and organizational viability (Amabile, 1996; Oldham \& Cummings, 1996; Shalley, 1991; Woodman, Sawyer \& Griffin, 1993; Zhou, 1998; Zhou \& Shalley, 2008). Creativity has also been said to be critical for social progress and economic growth (Florida, 2004; Schumpeter, 1939). Importantly, research findings have begun to provide support for these assertions, although the findings are still largely suggestive at this point (Gilson, 2008).

\section{Defining Creativity}

The terms creativity and innovation are often used interchangeably, but the academic literature makes a clear distinction between the two constructs. The most commonly accepted definition of creativity in the U.S. is the production of novel and useful ideas concerning products, services, processes, and procedures (e.g., Amabile, 1983, 1988, 1996; James, Clark, \& Cropanzano, 1999; Oldham \& Cummings, 1996; Runco, 1995; Shalley, 1991; Zhou, 1998) that are accepted within relevant domains (Ford, 1996). Creativity can be demonstrated by individual employees or work teams (Zhou \& Shalley, 2011). Further, employees in all types of jobs, in all functional areas, and at all levels of an organization have the potential to be creative at work (Amabile, 1996; Oldham and Cummings, 1996; Shalley, Gibson, \& Blum, 2000; Woodman, 
Sawyer, \& Griffin, 1993; Zhou, 1998). However, individual differences exist in terms of the magnitude of this potential (Zhou \& Shalley, 2011).

Regarding the difference between creativity and innovation, Zhou and Shalley (2011) assert, "whereas creativity emphasizes the production of new and useful ideas by individuals or teams, innovation emphasizes the implementation of new ideas or practices in a unit or throughout an organization" (p. 276). Thus, the primary difference is that creativity refers to ideas developed within the focal organization, whereas innovation can include the implementation of novel solutions or processes developed outside of the firm (Zhou \& Shalley, 2011).

Because the current study is based on the perceptions of Chinese participants, it is important to consider creativity in a Chinese context. While some scholars have asserted that China lags behind other countries in terms of creativity and innovation (Farmer, Tierney, \& Kung-McIntyre, 2003), Chinese societies strongly value creative efforts that are useful and practical (Gardner, 1989). Perceptions of novelty in Chinese settings are in large part defined by the culture and the context, as is the case in any cultural setting (Csikmentihalyi, 1999). Gardner (1988) offered the following definition of creativity specific to a Chinese setting, "the solution of problems in a way that is initially original but is ultimately accepted in one or more cultural settings." In both the Eastern and Western definitions of creativity, the core concept of new and practical ideas (i.e., novelty and usefulness) is paramount, although how novelty is applied may differ somewhat in China ( $\mathrm{Li}, 2012)$. It is also the case that most creativity theory development and research have been conducted in North America and Europe (Li, 2012). As an 
emerging force in the global marketplace, Chinese companies, and Chinese high-tech companies in particular, need to understand the antecedents of employee creativity in order to successfully market and sell their products to consumers around the world. Thus, the importance of researching creativity in Chinese contexts is relevant from a practitioner's standpoint as well as an academic one.

\section{Theoretical Foundations}

In developing the model for this study, both the motivational and the cognitive approach to researching the creativity of individuals, groups, and organizations were considered. The motivational approach has attracted the most research and has garnered an impressive number of findings (Shalley et al., 2004; Zhou \& Shalley, 2003). Before 2000 , most creativity research was conducted in the laboratory, guided by the motivational approach (Amabile, 1996; Shalley, 1991; Torrance, 1974). Recently, field studies have become more common and have provided increased insight into the processes of creativity in which employees take part. Taken as a whole, the literature shows that the variance in creativity explained by many creativity studies has ranged from the low to mid-teens and in general, has not exceeded .20. However, much more research must be done before stating that these effect sizes are conclusive. For comprehensive review articles on the topic, see the following recently published articles: Anderson, De Dreu, and Nijstad (2004); Shalley, Zhou, and Oldham (2004); and Zhou and Shalley (2003). In addition, Mumford (2011) recently edited a volume devoted to the theories of and empirical findings on creativity at work, titled the Handbook of Organizational Creativity. 
Componential theory of creativity. Within the motivational approach, the most commonly cited theory is Amabile's (1996) componential theory of creativity, which highlights the role of motivation in enhancing or reducing individuals' creativity. Amabile posited that three "components" must be present for an employee to exhibit high levels of creativity at work: 1) domain-specific talent, knowledge, and skills; 2) creativity-relevant skills and strategies; and 3) intrinsic motivation to be creative. The final component was theorized by Amabile to be most essential for creativity because it provides the energy or drive that activates and sustains the application of domain-specific talent, knowledge, and skills, as well as creative skills and strategies, toward creative production. In 2010, James and Taylor expanded Amabile's model by adding the role of goal setting and asserting that creativity can be directed toward positive or negative goals. Goals have been strongly and consistently shown to relate to both the levels of motivation that individuals experience and the purposes (i.e., productive outcomes) toward which motivation will be directed (Locke \& Latham, 2002; Mento, Steel, \& Karren, 1987). Figure 2 provides a visual depiction of the expanded componential theory of creativity, which includes affect-tinged goals, as well as positive and negative creative outputs as potential results of the creative process (see James \& Taylor, 2010 for a full explanation of this model).

In the present study, it is proposed that transformational leadership, especially the dimension of intellectual stimulation, serves as a motivating factor that inspires employees to set goals for increased creative performance. Organizational justice is also proposed to be a motivating factor because it serves as the bonding element that enables 
people to work together effectively (Cropanzano \& Greenberg, 1997). The research regarding organizational justice as a motivational theory states that when employees are treated fairly, they are intrinsically motivated to enhance performance (Zapata-Phelan et al., 2009). Diedendorff and Chandler (2010) proposed organizational justice and leadership are proximal external motivating influences in their integrative framework of motivation. Zapata-Phelan and colleagues (2009) found that procedural justice led to increased intrinsic motivation, which in turn led to increased performance.

Cognitive evaluation theory. Another theory that uses the motivational approach is Deci and Ryan's $(1980,1985)$ cognitive evaluation theory. This theory focuses on whether a factor that is external to an individual (i.e., a contextual factor) enhances or reduces that person's intrinsic motivation, depending on whether the factor is controlling or informational. If the factor is controlling, individuals are likely to perceive they are being pressured or constrained by external forces, and as a result, they are likely to have lower intrinsic motivation. Thus, controlling contextual factors are expected to decrease creativity. On the other hand, informational factors are likely to increase an individual's feeling of self-determination and competency, and as a result, the individual is likely to have high intrinsic motivation. Thus, contextual factors that are informational are likely to enhance creativity.

Interactional approach. The final theory to be reviewed here that guides the motivational approach to workplace creativity research is the interactional approach developed by Woodman, Sawyer, and Griffin (1993). It examines the complex interactions of contextual factors and individual differences to understand and predict 
creativity in the workplace. This theory asserts that the individual differences likely to contribute to variance in employee creativity include cognitive ability, personality, intrinsic motivation, domain-relevant knowledge, and positive or negative effects of previous experiences (Zhou \& Shalley, 2011). The contextual factors include leadership and management practices and the employee's relationships with his/her supervisor and co-workers.

While the motivational approach has guided most of the research on creativity at work, the cognitive approach has also made an impact, due in large part to the concept known as creative cognition. Creative cognition is a comprehensive term that refers to the creativity-relevant knowledge, skills, and processes necessary to produce creative outcomes (Finke, Ward, \& Smith, 1992; Smith, Ward, \& Finke, 1995). Its premise is that everyone has the ability to be creative, yet some individuals exercise their innate creative cognition more often and at higher intensities than others (Ward, Smith, \& Finke, 1999). There are many models of the cognitive creative process, but all of them include the following components: 1) identify a problem or opportunity, 2) gather information, 3) generate ideas, 4) evaluate ideas, and 5) select the ideas that merit further consideration and elaboration (Zhou \& Shalley, 2011). In this way, the cognitive approach defines creativity as an iterative process that can include reflection and action, experimenting, seeking feedback, and searching for new ways to do things.

In terms of integrating theoretical perspectives, the cognitive approach also relates to Amabile's creativity knowledge, skills, and abilities (KSA) component. Regarding creativity KSAs, some are highly cognitive in nature (knowledge), while some are 
learned psychomotor behaviors (skills). Abilities have a more innate component to them, but can also be developed, in part, through cognitive training.

\section{Theoretical Predictions of the Relationships between Diversity and Creativity}

The information processing perspective provides theoretical rationale regarding why diversity, in some cases, has been found to promote creativity and innovation. This perspective assumes that employees bring unique approaches and expertise to workrelated activities (i.e., task-oriented diversity), which can result in improved decisionmaking in a variety of areas. It posits that diversity in task-oriented attributes such as product knowledge or market expertise can provide teams with valuable information and unique approaches to generating solutions and solving problems. In the current study, this perspective provides some of the theoretical foundation regarding why employee's positive perceptions of diversity would lead to enhanced creative performance.

Social capital theory suggests that relations-oriented diversity also may promote creativity and innovation by providing more external connections through which individuals can obtain necessary knowledge and resources to generate novel and useful solutions (Oh et al., 2004). In the current study, in which employees working in technology firms are examined, social capital theory provides a reason why more positive perceptions of diversity climate may promote enhanced creative performance. Within technology firms, work within teams may be relatively autonomous to the work of other teams within the organization, but creative performance depends on staying connected to the rapid advancements in the field and to the needs of diverse markets. While the need for external connections is high, the need for smooth internal operations may be less 
important. Thus, social capital theory suggests that higher ratings of diversity climate would lead to positive outcomes - in this case, higher ratings of individual creative performance.

Some empirical findings have supported these theoretical arguments. These findings, as well as the hypotheses regarding the relationships among leadership, diversity, and creativity, are detailed in the next chapter. 


\section{Chapter 5: Prior Research and Hypotheses}

\section{Diversity Climate as a Mediator of the Leadership-Creativity Relationship}

The first main hypothesis of this study is that diversity climate mediates the relationship between transformational leadership and creative performance. A recent call by DeRue and colleagues (2011) provides impetus to propose and test the mediational mechanisms of transformational leadership. The theoretical predictions and/or empirical findings detailed below provide support for the following hypothesis:

Hypothesis 1. There will be a significant indirect effect of transformational leadership on individual creative performance through diversity climate.

Transformational leadership and diversity climate. While there is a lack of empirical research examining the relationship between transformational leadership and diversity climate, it is theoretically probable that the two constructs will be associated in a positive manner. Because transformational leaders act with integrity and inspire trust in employees, it is likely that the employees of these leaders who are different from each other on a variety of social categories (i.e., diverse) will perceive that the working environment is conducive to effective performance and that policies and procedures are implemented in a consistent manner. The likely result is that the employees of transformational leaders will tend to have more positive perceptions of diversity climate. Likewise, when transformational leaders provide individual consideration to employees, and employees both experience this personalized attention and see the leader treat all employees in the same manner, it is likely that employees would perceive a healthier 
diversity climate. In support of this logic, Wieland (2004) found that transformational leadership helped to create more positive diversity climates in organizations. Leaders should also be proactive in building a positive diversity climate. That is, they should proactively demonstrate the extent to which they value diversity and inclusion by taking action to ensure that employees feel socially integrated into their work groups and the organization as a whole and that policies and practices are fair and are implemented consistently in all situations and across employees of different backgrounds. Thus, the following hypothesis is proposed:

Hypothesis 1a: Transformational leadership will be significantly and positively related to perceived organizational diversity climate.

Transformational leadership and creative performance. Much of the research guided by the motivational approach has focused on contextual factors that demonstrate different associations with creative performance than with routine task performance. One of the most salient contextual factors that impacts creative performance is the leadership and managerial behaviors exhibited by employees' supervisors (Amabile \& Conti, 1999; Amabile, Conti, Coon, Lazenby, \& Herron, 1996; Amabile \& Gryskiewicz, 1989; Amabile, Schatzel, Moneta, \& Kramer, 2004; Andrews \& Farris, 1967; Frese, Teng, \& Wijnen, 1999; George \& Zhou, 2001; Oldham \& Cummings, 1996; Shalley \& Gilson, 2004; Shin \& Zhou, 2003; Tierney \& Farmer, 2002, 2004). In addition, creativity researchers have reached a consensus that leadership plays an important role in facilitating and promoting employee creativity (Hirst, van Knippenberg, \& Zhou, 2009; Shalley, Zhou, \& Oldham, 2004). Following from Deci and Ryan's (1980, 1985) 
cognitive evaluation theory, leadership behaviors that are informational are likely to lead to sustained or increased employee motivation, thus more likely enhancing creativity performance.

In addition to general leadership behaviors, feedback and evaluation have been studied as contextual factors in the promotion of employee creativity. The research demonstrates that when feedback is perceived to be controlling, employee creativity decreases (Amabile, 1979; Amabile, Goldfarb, Brackfield, 1990; Bartis, Szymanski, \& Harkins, 1988; Cheek \& Stahl, 1986; Shalley \& Perry-Smith, 2001; Szymanski \& Harkins, 1992; Zhou, 1998). Conversely, when feedback or supervisory evaluations are perceived to be informative and for developmental purposes, creativity appears to be facilitated (Shalley, 1995; Zhou, 1998; Zhou \& Oldham, 2001). Further, Shalley and Perry-Smith (2001) found lower creativity for individuals who anticipated a judgmental evaluation compared to those who expected a developmental evaluation. Providing appropriate information and developmental feedback to one's employees could reasonably be described as a critical characteristic of the individualized consideration dimension of transformational leadership.

Creativity has been found to be highest when an employee self-identifies as a creative person (i.e., has a strong creative role identity) and perceives that the employing organization values creative work (Farmer, Tierney, \& Kung-McIntyre, 2003). Simply communicating that employees are expected to be creative can be a catalyst for creative performance (Ford, 1996). Intellectual stimulation is in part described as inspiring employees to be creative and to think outside the box. Through this dimension of 
transformational leadership, it is theorized that leaders can demonstrate a strong organizational value for creativity, and thus raise levels of employee creativity.

Motivational orientation may be partially shaped by the environment (Amabile, 1983), which can be influenced by transformational leadership. Specifically, the inspirational motivation dimension can enhance employees' intrinsic motivation. Intrinsic motivation has been positioned as playing a crucial role in employee creativity (Tierney, Farmer, \& Graen, 1999). Somech (2006) conducted a study of 136 primary care teams and found functionally diverse teams with participative leaders engaged in more team reflection, which in turn was associated with team innovation. Participative leadership is conceptually similar to inspirational motivation.

In support of these theorized similarities between the research findings on effective leadership behaviors and the dimensions of transformational leadership, Shin and Zhou (2003) found evidence of a positive relationship between transformational leadership and creativity $\left(\Delta R^{2}=.05, p<.01\right)$. An employee's intrinsic motivation partially explained the positive relationships. In light of the arguments presented above and the research findings, it is expected that leaders who demonstrate transformational leadership provide the motivation, vision, and specific feedback necessary for individual employees to display a higher level of creative performance that those employees with leaders who do not.

Hypothesis 1b: Transformational leadership will be significantly and positively related to individual creative performance. 
Diversity climate and creative performance. In general, positive social climates and feelings of security tend to promote positive emotional states and positive goals (James \& Taylor, 2010). It is likely that creativity should follow from these emotional and goal effects, and research has indicated that more supportive organizational environments yield higher positive creativity in the workplace (Amabile, et al., 1996; Tesluk, Farr, \& Klein, 1997; Ford, 1999). One of the main aspects of diversity climate is the degree to which all employees are socially integrated, so it stands to reason that coworkers are a social contextual factor with the potential to shape employee creativity (Woodman et al., 1993). Recent studies have shown that co-workers influence creativity through encouragement, support, open communication, and informational feedback (Amabile et al., 1996; Madjar et al., 2002; Zhou \& George, 2001). Shin and Zhou (2003) emphasize the importance of studying leader behavior in concert with follower perceptions and beliefs, rather than focusing solely or mostly on leader behavior alone in creativity research. While scholars have commonly predicted a significant and positive relationship between diversity climate and employee creativity, there is a lack of empirical evidence for this assertion. However, based on the theoretical arguments and the research findings presented above, the following hypothesis is proposed:

Hypothesis 1c: Diversity climate will be significantly and positively related to individual creative performance.

\section{Interaction of Transformational Leadership and Diversity Climate}

The second main hypothesis of this study is that transformational leadership and diversity climate are two contextual factors that will interact in a multiplicative way to 
impact the other variables in the model. The following theoretical predictions and/or empirical findings provide support for this assertion:

Hypothesis 2: Transformational leadership and diversity climate will interact to significantly affect individual creative performance, such that diversity climate will have a stronger relationship to creative performance when transformational leadership is high than when transformational leadership is low.

The existing research indicates that an organization's diversity climate plays a critical role in many important outcomes, such as training transfer (Rynes \& Rosen, 1995 ) and intention to accept a position (McKay \& Avery, 2006). It has also been shown to predict behavioral outcomes, such as attendance (Avery, McKay, Wilson, \& Tonidandel, 2007). There is evidence that employees who share similar perceptions of diversity climate may share other attitudes, such as job satisfaction and organizational commitment (Nishii \& Raver, 2003). The same can be said for transformational leadership in terms of its ability to predict organizational outcomes and employee behavior (Barling et al., 2011; Judge \& Piccolo, 1994). Thus, in this study, transformational leadership and diversity climate are conceptualized as broad contextual factors that influence the perceptions of employees. As such, they are proposed to be more distal variables in the model, as compared to the proximal variables of organizational justice and organizational identity.

The concept of organizational culture is closely linked to that of organizational climate. Empirical studies that examined the effects of dissimilarity in organizations with differing cultures seem to support the general argument that organizations with cultures 
that reflect the value of diversity are more likely to realize the potential benefits of workplace diversity (Dass \& Parker, 1999; Ely, 2004; Ely \& Thomas, 2001; Gilbert \& Ivancevich, 2000). On the other hand, organizational cultures that endorse a so-called color-blind approach, in which individual differences are not acknowledged and employees' different needs, assets, and perspectives are disregarded, may reinforce majority dominance and result in disengagement by minority employees and women (Plaut, Thomas, \& Goren, 2009). At the same time, the extant literature does not provide clear guidance on how to establish appropriate cultures that enhance the likelihood of leveraging the benefits of diversity. It is generally accepted that top management has the responsibility to set the tone of an organization's culture (Wasserman, Gallegos \& Ferdman, 2008), but empirical research has not yet delineated the particular actions leaders should take and the behaviors they should engage in to succeed in leading their diverse organizations.

Many studies have examined the main-effect outcomes of transformational leadership (Bass, Avolio, Jung, \& Berson, 2003; Lim \& Ployhart, 2004; Schaubroeck, Lam, \& Cha, 2007); however, there is a need for research that investigates whether it moderates the relationship of diversity climate and creative performance and, if it does, which process(es) mediate that effect (Kearney \& Gebert, 2009). Research demonstrates that workplace diversity has the potential to bring about organizational benefits and detriments (Hülsheger et al., 2009; Jackson \& Joshi, 2011; Stewart, 2006). Employers must make the effort to ensure that the knowledge, skills, and abilities of all employees 
are fully utilized to achieve organizational goals. Leaders play a vital role in facilitating this process (Hogan \& Kaiser, 2005).

Diversity climate is conceptualized as a broad contextual factor that interacts with transformational leadership to influence creative performance, as well as the other dimensions of diversity. When employees perceive that organizational leaders authentically prioritize recruiting, hiring, and retaining a diverse workforce, as well as developing an inclusive working environment, employees are more likely to reap the benefits of diversity, and thus, be motivated to perform in more creative or innovative ways. When the shared perception among employees is that leaders prioritize developing a diverse and inclusive workforce and all employees are adequately socially integrated into teams and the organization as a whole (i.e., a strongly positive diversity climate), the conditions for eliciting the creative potential of individuals will be realized and creative performance will increase. The positive interaction of transformational leadership and diversity climate on individual creative performance would indicate that transformational leaders emphasize the value of diversity to the extent that employees perceive it, which in turn enhances employee creativity and innovation.

However, only three empirical studies have thus far examined the link among diversity, leadership, and performance outcomes. In a longitudinal study of 62 research and development teams of a German pharmaceutical company, Kearney and Gebert (2009) examined transformational leadership as a moderator of the relationship of age, nationality, and educational background diversity with team outcomes. They found that the positive relationship between team nationality and educational diversity and leader 
ratings of team performance was stronger for teams with transformational leaders, compared to teams whose leaders were not perceived to be transformational. Age diversity was not related to team performance when transformational leadership was high, and it was negatively related to team performance when transformational leadership was low. This study suggests that transformational leaders more effectively facilitated the exchange and use of task-related information, which contributed to the teams' performance. In addition, Shin and Zhou (2007) have shown that transformational leadership moderates the relationship between diversity on educational specialization and creativity such that this relationship is more positive when transformational leadership is high rather than low. The findings of these studies highlight the importance of examining the effects of the interaction of transformational leadership and diversity on creativity performance.

The current study builds on, extends, and differs from these studies in several ways. Kearney and Gebert (2009) only looked at objective types of diversity, whereas this study examines the subjective perceptions of employees regarding three dimensions of workplace diversity (i.e., diversity climate, organizational justice, identity). In addition, instead of using task performance as a dependent variable, creative performance is the outcome of interest, which is highly valued in high-tech firms and more theoretically tied to diversity. In addition, the current study focuses on three dimensions and four types (as control variables) of diversity, whereas Shin and Zhou (2007) examined only informational diversity. Instead of proposing transformational leadership as a moderator of the relationship between categorical diversity and creativity, this study 
focuses on diversity climate from among the inter-connected web of constructs in the nomological network of workplace diversity as a situational factor that interacts with transformational leadership to influence organizational justice and organizational identity, and ultimately, creative performance. In this model, the impact of transformational leadership and diversity climate are predicted to interact in a multiplicative manner, rather than an additive manner, as prior research has conceptualized and tested this relationship. Thus, the current study takes a broader and more complex view of workplace diversity as a multi-dimensional construct than any of the previous studies of transformational leadership, diversity, and creativity.

As detailed previously, the empirical findings suggest that the mere presence of diversity does not guarantee an increase of creativity and innovation. Rather, the research demonstrates that workplace diversity has the potential to result in both positive and negative outcomes. Furthermore, the types and dimensions of diversity under examination make a difference in terms of important organizational outcomes. The faultlines perspective sheds light on the mixed findings regarding the relationship between diversity and creativity, while at the same time illuminating the importance of leadership. When the presence of diversity inadvertently results in the creation of strong faultlines between groups or cultures, it may interfere with, rather than support, effective problem solving (Jackson \& Joshi 2011). Workplace diversity may facilitate positive creativity to the extent to which mechanisms, such as strong organizational norms promoting positive interactions among different others, also exist for preventing substantial inter-group polarizations (James \& Taylor, 2010; Osche, 1990; Simonton, 
1995). Leaders are vital to developing these types of norms. The strength of systems or strategies of social coordination and integration developed by leaders should, therefore, interact with perceptions of diversity climate to influence the level of employee creative performance. To prevent or decrease the development of fault lines, organizational leaders should proactively communicate a strong vision for employees to work toward together, challenge employees to think critically, and attend to conflict management and communication skills of team members (Jackson \& Joshi, 2011). These behaviors are characteristic of the inspirational motivation, intellectual stimulation, and individualized consideration dimensions of transformational leaders. It is predicted that strongly positive perceptions of diversity climate along with leader behaviors that result in the effective and consistent implementation of systems and procedures designed to promote positive interactions and intergroup cooperation will promote individual employee creativity. In summary, the extant research points to a potential moderation effect between transformational leadership and diversity climate in fostering the conditions that enable employees to demonstrate creativity in the workplace.

It is argued that the individual creative performance will depend on the extent to which both supervisors are believed to demonstrate those behaviors characteristic of transformational leadership and the extent to which diversity climate is perceived to be positive, supporting Hypothesis 2, stated above.

\section{Organizational Justice in the Moderated Mediation Model}

The third main hypothesis of this study is that organizational justice mediates the relationship between the interaction of transformational leadership and diversity climate 
and creativity. The following theoretical predictions and/or empirical findings provide support for this assertion:

Hypothesis 3. There will be a significant indirect effect of the interaction of transformational leadership and diversity climate on individual creative performance through organizational justice.

Transformational leadership and organizational justice. One of the hallmarks of transformational leadership is its emphasis on building employee trust. A transformational leader must demonstrate a high level of integrity (Bird \& Osland, 2004) in order to build a strong and broad foundation of employee trust. Studies have shown that trust in the leader is positively related to leader fairness (van Knippenberg, De Cremer, \& van Knippenberg, 2007). In addition, Pillai et al. (1999) used a path model to show that transformational leadership behaviors lead to increased perceptions of organizational justice, which ultimately leads to higher performance. Based on this empirical evidence, the following hypothesis is proposed:

Hypothesis 3a: Transformational leadership will be significantly and positively related to perceived organizational justice.

Diversity climate and organizational justice. With diversity climate defined as a broad contextual factor that includes social integration of employees as well as fair policies and practices, it stands to reason that employee perceptions of diversity climate are expected to be significantly and positively related to employee perceptions of organizational justice. In their review of work team diversity, Jackson \& Joshi (2011) assert, "the evidence indicates that employers may increase the likelihood of leveraging 
the potential benefits of diversity by inspiring positive diversity climate perceptions through the use of fair human resource management practices" (p. 673). Scholars have also noted the conceptual similarities of diversity climate and organizational justice (McKay, Avery \& Morris, 2008); however, there are important differences and the two concepts have been empirically distinguished using confirmatory factor analysis (McKay et al., 2007). Specifically, organizational justice only refers to employees' perceptions of fairness, while diversity climate encompasses the components of structural and social integration necessary for employees to perceive that organizational leaders truly value diversity and inclusion. Past research has shown that diversity climate is associated with all four forms of justice (i.e., distributive, procedural, interpersonal and informational; Rupp, Bashur, \& Liao, 2007; Cropanzano, Li, \& James, 2007). If employees perceive the overall climate is fair and inclusive (i.e., positive perceptions of diversity climate), it follows that employees would also have positive evaluations of their employer's level of organizational justice. While there are many associated outcomes of a positive evaluation of diversity climate, it is likely that fairness at work is a significant one.

Hypothesis 3b: Diversity climate will be significantly and positively related to perceived organizational justice.

\section{Interaction of transformational leadership and diversity climate and}

organizational justice. Given the research stated above and the conceptualization of transformational leadership and diversity climate as broad contextual factors that interact in a multiplicative way, the following hypothesis regarding the way in which this interaction influences organizational justice is proposed. 
Hypothesis 3c: Transformational leadership and diversity climate will interact to

significantly influence perceived organizational justice, such that diversity

climate will have a stronger relationship to organizational justice when

transformational leadership is high than when transformational leadership is low.

Organizational justice and creative performance. Fairness at work has been

found to build trust and organizational commitment, improve job performance, and foster organizational citizenship behaviors (Cropanzano et al., 2007). Colquitt and colleagues (2012) recently found that organizational justice is related to job performance through employee trust. James and colleagues (Clark \& James, 1999; James \& Clark, 2009) studied the effects of fair treatment on creativity and found that people who were treated fairly tended to demonstrate increased creativity directed toward positive ends. The study results indicate that perceived fair treatment seems to facilitate creativity goals and some distinctive creativity thinking skills. Following from Deci and Ryan's $(1980,1985)$ cognitive evaluation theory, leader or co-worker behaviors that are informational are likely to lead to sustained or increased employee motivation, thus creativity at work is likely to increase. Informational justice is one of the four dimensions of organizational justice (Colquitt et al., 2001). Thus, it is likely that organizational justice will lead to increased employee creative performance. Given the above rationale and empirical evidence, the following hypothesis describes the predicted relationship of organizational justice as a dimension of the workplace diversity taxonomy and individual creative performance. 
Hypothesis 3d: Organizational justice will be significantly and positively related to individual creative performance.

Hypothesis 3e: There will be a significant indirect effect of diversity climate on individual creative performance through organizational justice.

\section{Organizational Identity in the Moderated Mediation Model}

The fourth main hypothesis of this study is that organizational identity mediates the relationship between the interaction of transformational leadership and diversity climate and creativity. The following theoretical predictions and/or empirical findings provide support for this assertion:

Hypothesis 4. There will be a significant indirect effect of the interaction of transformational leadership and diversity climate on individual creative performance through organizational identity.

Transformational leadership and organizational identity. Effective diversity management is likely to foster greater organizational identification among a diverse group of employees because they are more likely to perceive that organizational leaders have the best interests of all employees in mind, rather than perceiving that a small subset of employees is favored. When employees perceive that leaders care about them, in equal measure to their co-workers, organizational identity tends to develop. Strong organizational identity, in turn, compels employees to view the organization's fate as their own and to act in ways that contribute positively to the organization (Hogg \& Terry, 2000). Transformational leader behaviors may increase employee motivation in a diverse workforce by building linkages between team members' self-concepts and the team or 
organization's work, thus increasing identification with the team (Ellemers, De Gilder, \& Haslam, 2004; Turner \& Haslam, 2001). By articulating the mission and vision of the organization (i.e., inspirational motivation) and appealing to the values that employees have in common with the organization, the interests of individual employees are linked with that of the organization (Kark \& Shamir, 2002). Further, transformational leadership has been found to be positively related to identification with the leader and the group overall (Kark, Shamir \& Chen, 2003). Given the aforementioned theoretical predictions and research findings, it is expected that transformational leadership will lead to higher employee perceptions of organizational identity.

Hypothesis 4a: Transformational leadership will be significantly and positively related to perceived organizational identity.

Diversity climate and organizational identity. When employees feel socially integrated into work teams and perceive that their organization values a variety of social backgrounds, life and work experiences, and perspectives, they tend to develop an increased sense of identity with the organization as a whole. In other words, the message that employees receive from a positive organizational diversity climate is that there is a common organizational identity (e.g. Brewer, 1991; Koper et al., 1993) that includes everyone, and as a result, employees are more likely to take on that identity as their own. Thus, the following hypothesis is proposed:

Hypothesis 4b: Diversity climate will be significantly and positively related to perceived organizational identity. 


\section{Interaction of transformational leadership and diversity climate and identity.}

Given the research stated above and the conceptualization of transformational leadership and diversity climate as broad contextual factors that interact in a multiplicative way, the following hypothesis regarding the way in which this interaction influences organizational identity is proposed.

Hypothesis 4c: Transformational leadership and diversity climate will interact to significantly influence perceived organizational identity, such that diversity climate will have a stronger relationship to organizational identity when transformational leadership is high than when transformational leadership is low.

Organizational identity and creative performance. When employees feel like they are an integral part of the team, they are more likely to share ideas freely, which can lead to creativity. Thatcher and Greer (2008) found that when team members know the relative importance of an individual's identity, it positively relates to the individual's creativity. The authors argued that the more an employee feels known and understood, the more likely they are to bring to bear the entire repertoire of their experiences, knowledge, and skills for the task at hand. In addition, a recent study found that meaningfulness in the workplace is positively related to supervisor ratings of employee creativity via organizational identification and positive psychological experiences (Cohen-Meitar, Carmeli \& Waldman, 2009). This study seeks to provide further empirical evidence regarding the relationship between identity in the context of a diverse workplace and employee creativity. 
Hypothesis 4d: Organizational identity will be significantly and positively related to individual creative performance.

Organizational justice and identity. Similarly, organizational justice is expected to significantly and positively predict employee perceptions of organizational identity, and this assertion has been supported in the literature (Mor Barak, 2011). With the noted conceptual similarities of diversity climate and organizational justice, this assertion is not a surprise. Specifically, Johnson and Lord (2010) conducted a laboratory experiment and found that interdependent and individual self-identities were higher when individuals experienced fairness and unfairness, respectively. They found effects occur at both the implicit and explicit levels, but they were stronger in the former case. In addition, a recent study has shown that three forms of supervisory justice (procedural, interactional, and distributive) leads to increased group identification (Lipponen, Wisse, \& Perala, 2011). In the quantitative study of the workplace diversity taxonomy, Taylor and James (2013) found that the organizational justice dimension was most highly correlated with the identity dimension of the taxonomy $(r=.59, p<.01)$. When procedures are fair, it conveys the message that employees have a common organizational identity (e.g. Brewer, 1991; Koper et al., 1993). Thus, based on the above evidence, the following hypotheses are proposed:

Hypothesis 4e: Organizational justice will be significantly and positively related to perceived organizational identity. Hypothesis 4f: There will be a significant indirect effect of diversity climate on individual creative performance through organizational justice. 


\section{Contributions to the Literature}

While some of the main effects hypotheses described above have substantial support in the literature, others do not have as much, or any, empirical support. Thus, this study will substantially contribute to the literature regarding the following hypotheses, which have relatively less initial evidence:

1a. Transformational leadership will be significantly and positively related to perceived organizational diversity climate.

1b. Transformational leadership will be significantly and positively related to individual creative performance.

1c. Diversity climate will be significantly and positively related to individual creative performance.

3a. Transformational leadership will be significantly and positively related to perceived organizational justice.

4a. Transformational leadership will be significantly and positively related to perceived organizational identity.

4b. Diversity climate will be significantly and positively related to perceived organizational identity.

Testing the mediation hypotheses will also contribute significantly to the

literature, as will the mediated moderation hypotheses.

Employee information and ratings regarding six control variables (age, education, gender, organizational tenure, proactive personality, and openness to experience) were collected to test for likely alternative explanations for variations in creative performance as suggested by previous research (e.g., Oldham \& Cummings, 1996; Tierney \& Farmer, 2002). Openness to experience and proactive personality are well-established predictors of individual creativity. 


\section{Chapter 6: Method}

\section{Participants}

Employees. The participants of this study were 418 employees of three information-technology service and manufacturing companies based in Beijing and Shenzhen, China. All participants were Chinese nationals. The surveys used in the study were sent to 545 information-technology manufacturing or development workers, all of whom held an undergraduate degree or higher. At the end of the third wave of data collection, 356 employees provided self-ratings of their creative performance, resulting in a response rate of $65.32 \%$, and 371 supervisors provided ratings of creative performance for each of their direct reports, resulting in a response rate of $68.07 \%$. The participants were predominantly male $(\mathrm{N}=318,76.08 \%)$, and their average age was 29.36 years. Participants' average organizational tenure was three years and nine months, and they had an average of four years and seven months of education after college. Table 7 provides the means and standard deviations of the demographic variables measured in this study, including age, education, gender, and organizational tenure.

Supervisors. The 64 immediate supervisors of the employees in the main sample also participated by providing ratings of the creative performance of each of their direct reports. On average, each supervisor oversaw the work of six employees, which was the average number of direct report-creative performance ratings provided by each supervisor. Since the data collection was tied into the annual performance appraisal, the response rate of the supervisors was $100 \%$. 
For the projected analyses, a minimum sample size of 244 individuals is needed for 10 variables: six control variables (organizational tenure, age, education, gender, proactive personality, and openness to experience) and four independent variables (transformational leadership, diversity climate, organizational justice, and organizational identity). That estimate was based on a small-medium effect size $\left(f^{2}\right)$ of .05 and power goal of .80 . Thus, the sample size at the end of the third wave of data collection (i.e., $\mathrm{N}=$ 371 for supervisor ratings, and $\mathrm{N}=418$ for employee self-ratings of creative performance) is adequate for the data analyses conducted.

\section{Procedure}

The data for this study was collected in three waves from employees (with a subset of different constructs, as outlined below, measured at each wave) and their supervisors (assessment of direct report creative performance, measured at Wave 3). The

first wave survey had the control variables listed above, including demographic and dispositional variables, as well as employee perceptions of the transformational leadership behaviors of their supervisors. The second wave survey had the measures of diversity climate, organizational justice and identity, and the third wave had the measures of employee self- and supervisor-rated individual creative performance. An overview of the measures by source and data collection timing is provided in Table 2. Because the survey items were originally developed in English, all items were back-translated and recentered following procedures detailed by Brislin $(1986 ; 1993)$. That is, the original English-language versions of the construct measures were translated into Mandarin by a 
psychology professor in Beijing, and then checked for accuracy by a Chinese graduate student who was also fluent in both languages.

The questionnaires were sent to employees (including the supervisors) via the human resources department of each company with a cover letter assuring that participation is voluntary, employee responses would be kept confidential, and that the data would be used for academic purposes only. The second wave survey was conducted with the same group of employees approximately five weeks after the first wave was sent, and the third wave data were collected roughly six weeks later during the annual performance appraisal period of the participating companies. The supervisor ratings of creative performance were collected during the third wave. The human resources department director informed the supervisors that they needed to provide the ratings of their direct report's creative performance. The ratings were combined with the company's annual performance appraisal, which led to a supervisor response rate of $100 \%$. Therefore, the time lag between each wave ranges from five to six weeks.

\section{Measures}

Control variables. The demographic control variables are the following: gender, age, organizational tenure and educational level. While the first three variables were measured using standard demographic survey questions, educational level was measured by asking participants to state the number of years of education they had after college. In addition, two dispositional variables were measured: proactive personality and openness to experience. Proactive personality was assessed using 10 items from Seibert, Crant, and 
Kraimer's (1999) scale. The openness to experience dimension of the Big Five was assessed using the eight items of Saucier's (1994) subscale.

Predictors and outcomes. The primary variables in the study (i.e., the predictors of the outcome measures of transformational leadership, diversity climate, organizational identity, organizational justice, and individual creative performance) were collected via assessments of the perceptions of focal employees. Responses for all items were given on a 7 -point scale $(1=$ strongly disagree to $7=$ strongly agree $)$.

Transformational leadership. Ten items were used to assess this construct, which were adapted from Transformational Leadership Behavior Inventory (Podsakoff, MacKenzie, \& Bommer, 1996) and the Multifactor Leadership Questionnaire (Bass \& Avolio, 1989). Two of the four dimensions of transformational leadership were measured (i.e., inspirational motivation and intellectual stimulation), since charisma/idealized influence and individualized consideration were not part of the conceptual model of creative performance that guided the study. The inspirational motivation and the intellectual stimulation subscales were measured using five items each. The inspirational motivation subscale has been reported to have an internal consistency of .94, using Cronbach's alpha (James \& Lahti, 2011; James, Yao, \& Lahti, in press). An example of an item in this subscale includes, "My manager inspires others with his/her plans for the future." The intellectual stimulation subscale has been reported to have an internal consistency of .82 (James \& Lahti, 2011). An example of an item in this subscale includes, "My manager stimulates individuals to think about old problems in new ways." 
Using a 7-point scale, respondents indicated the extent to which they agreed with each statement regarding their immediate supervisor.

Diversity climate. To measure diversity climate, the Workplace Diversity Inventory (WDI; Taylor, James \& Murry, 2012), was used. The subscale has demonstrated good internal consistency ( $\alpha=.82$; Taylor et al., 2012). In addition, evidence for structural validity was found with good to excellent model fit (Taylor et al., 2012). An example of an item in the diversity climate subscale includes, "Leaders here connect diversity to the organization's mission and vision."

Organizational justice. Similar to the construct above, organizational justice was measured using the Organizational Justice subscale of the Workplace Diversity Inventory (WDI; Taylor, James \& Murry, 2012), which has shown high reliability ( $\alpha=.91$; Taylor et al., 2012). As noted above, evidence has been found for the construct validity of this measure (Taylor et al., 2012). An example of the items in the WDI Organizational Justice subscale includes, "People at work are treated fairly regardless of who they are."

Organizational identity. A subscale of the WDI (Taylor, James \& Murry, 2012) was also used to measure organizational identity. For this subscale, evidence was found for structural validity, with good to excellent model fit (Taylor \& James, 2012). One example item in the subscale is, "I feel a strong sense of belonging at my organization."

\section{Employee self-rated and supervisor-rated individual creative performance.}

The measure of individual creative performance used for both supervisor ratings and employee self-ratings was developed by Zhou and George (2001). While the measure was nearly identical, the items and instructions differed slightly to make sense for the 
different sources of ratings. The measure is a 13-item instrument, which has

demonstrated internal reliability of .97 . On a 7-point scale ranging from 1, "very

uncharacteristic,'” to 7, "very characteristic,'” employees self-rated how characteristic

each of 13 behaviors was for themselves on the job, and supervisors rated how

characteristic each behavior was for each of the employees who they supervise and who participated in the study. A sample item of this scale is, "I (or my employee) come(s) up with new and practical ideas to improve performance."

\section{Analyses}

Confirmatory factor analyses. For each variable included in the analyses, a single-level and multi-level confirmatory factor analysis (CFA) was conducted using Mplus version 5.21 (Muthén \& Muthén, 2010) to check for unidimensionality and to examine construct validity for the measures. To examine model fit, chi-square values and corresponding degrees of freedom are reported. Since chi-square values are sensitive to sample size, the values of the alternative fit indices are also reported, including the Root Mean Square Error of Approximation (RMSEA), Standardized Root Mean Square Residual (SRMR), with values below .06 and .08, respectively, employed as indicators of good model fit (Hu \& Bentler, 1999). In addition, the Comparative Fit Index (CFI) and the Tucker Lewis Index (TLI) are reported, with values above .95, respectively, employed as indicators of acceptable model fit (Hu \& Bentler, 1999).

Testing for dependence. To investigate possible confounding organization- and supervisor-level mean differences among the three companies in which the data was collected and among the 64 supervisors who rated their direct reports, the intraclass 
correlations (ICC [1]) were calculated and examined to determine whether there was nesting within organizations and supervisors, indicating the errors were correlated and the assumption of independence of errors was violated. It is important to determine the amount of dependence within the data because it can lead to inflation of Type I error rates (rejection of a true null hypothesis). ICC (1) compares the between-organization (and between supervisor) sum of squares to the total sum of squares, based on the results of a one-way ANOVA, in which organizations, then supervisors, are the independent variable. ICC (1) values range from zero to one and represent the proportion of variance in individuals' perceptions accounted for by differences in organizations and supervisors. In general, ICC (1) values have ranged from 0 to .5 , with a median of .12 (James, 1982). However, even ICC values of .01 can inflate Type I error rates (Barcikowski, 1981).

Hypothesis testing. Due to the results of the tests for dependence, both single and multilevel path analyses using Mplus version 5.21 (Muthén \& Muthén, 2010) were conducted to test the model using employee self-rated creative performance (ECP) as the outcome variable (ECP model) and for the model with supervisor-rated creative performance (SCP) as the outcome variable (SCP model). Due to the complexity of the model, composites of each variable were calculated and used to test both models. The models were fully saturated, and the chi-square, degrees of freedom, and alternative fit indices are reported to confirm this level of saturation. For the single-level models, the standardized factor loadings are reported, while the unstandardized factor loadings are reported for the multi-level models. 
Multilevel modeling estimates regression coefficients with adjustments for nonindependent data. It attempts to quantify the amount of interdependency in the data and permits prediction of individual scores adjusted for group differences and prediction of group scores adjusted for individual differences within groups. Unequal sample sizes are not necessarily a problem, which was helpful for this analysis, since the number of people in each of the 64 groups ranged from three to 18 employees.

The first-level predictors (transformational leadership, diversity climate, organizational justice, and organizational identity) and personality control variables (proactive personality and openness to experience) were centered by subtracting the mean of each variable from each employee's composite score. The variables were centered to reduce concerns regarding multicollinearity and to aid in the interpretation of the results. Because these variables were centered for the multilevel path analyses, the intercept can be interpreted as the mean level of creative performance for a person at the mean level of transformational leadership, for example, controlling for group membership, rather than when transformational leadership equals zero.

The demographic control variables (i.e., age, gender, education, organizational tenure) were group mean centered (using team means) in both the single-level and multilevel analyses to remove team-level effects from these individual-level variables. In the multi-level models, the group mean centered demographic control variables were used to account for team-level variance of these variables.

To create the team-level predictors, grand mean centering was employed. First, the individual scores were transformed into their team mean scores. Next, the grand mean 
of the team means was calculated for each variable. Lastly, the team-level variables were centered by subtracting the grand mean from the team scores, resulting in 64 grand mean centered values of each predictor and demographic control variable to use as the teamlevel predictors and controls in the multilevel analyses. Results were examined for positive and significant beta weights. 


\section{Chapter 7: Results}

\section{Confirmatory Factor Analyses}

Tables 3 and 4 provide the chi-square values, degrees of freedom, and the values of the alternative fit indices for the single-level and multilevel confirmatory factor analyses (CFAs) conducted to examine the construct validity of the variables measured with more than one item. A single-level CFA indicates modeling only at the individuallevel, while a multilevel CFA indicates modeling at both the individual and team (or group) level.

Transformational leadership. Transformational leadership was theorized and tested to be a second-order factor with two first-level factors (inspirational motivation and intellectual stimulation). However, since a model with only two first-level factors would not converge in a second-order factor model tested using Mplus, CFAs were conducted for four different models using the items of transformational leadership and organizational justice, with the two best fitting models being the closest to resemble the theorized factor structure of transformational leadership and organizational justice (see Table 5). Taken together, the alternative fit indices suggested acceptable model fit for the second-order theorized factor model $\left(\right.$ Model 3: $\chi^{2}(74)=252.41, p<.001$, RMSEA $=$ $.08, \mathrm{SRMR}=.05, \mathrm{CFI}=.95$, and TLI $=.94 ;$ Model $4: \chi^{2}(72)=252.41, p<.001, \mathrm{RMSEA}$ $=.08, \mathrm{SRMR}=.05, \mathrm{CFI}=.95$, and $\mathrm{TLI}=.93)$. Table 5 presents the chi-square values, degrees of freedom, and fit indices for each of the four comparative CFA models of transformational leadership. 
Workplace Diversity Inventory. The Workplace Diversity Inventory (WDI) was theorized and tested to be multi-dimensional, with three factors representing each of the following constructs: diversity climate, organizational justice and organizational identity. Evidence has been found to support this factor structure (Taylor, James, \& Murry, 2012); however, since the scale was developed only recently using a mostly U.S. sample, it was necessary to test the construct validity of the WDI scale in a Chinese context. Thus, two models were tested using single-level and multilevel CFAs to determine whether the theorized three-factor model fit the data better than a one-factor model (see Table 6). The multilevel CFA indicated that the three-factor model provided the best model fit for the data and the fit was good (SCP Model: $\chi^{2}[134]=295.11, p<.001$, RMSEA $=.05$, SRMR $=.05$ [within], CFI $=.95$, and TLI $=.94)$.

Diversity Climate. Measured with five items, diversity climate was theorized to be unidimensional. A single-level CFA was conducted to test for unidimensionality, and the fit indices suggested poor model fit $\left(\chi^{2}[5]=122.70, p<.001, \mathrm{RMSEA}=.24\right.$, SRMR $=.04, \mathrm{CFI}=.93$, and TLI $=.86$ ). Thus, a multilevel CFA was conducted, and the model fit improved but remained questionable $\left(\chi^{2}[15]=126.12, p<.001, \operatorname{RMSEA}=.13\right.$, SRMR $=.05, \mathrm{CFI}=.94$, and TLI $=.91$ ). To further examine the factor structure of this variable, the standardized loadings were examined and all loadings were above .83 (item $1=.84$, item $2=.84$, item $3=.89$, item $4=.85$, item $5=.85$ ). Since all of the items loaded saliently onto one factor, none were deleted from the CFA. To further examine factor structure, an exploratory factor analysis using principle axis factoring in SPSS was conducted to explore the dimensionality of the five items of the WDI diversity climate 
scale in light of the questionable model fit indicated by the multilevel CFA. The results of the EFA indicated there was one only factor with an eigenvalue over 1 . This factor had an eigenvalue of 3.92 , which accounted for $78.30 \%$ of the variance in the responses to the five diversity climate items. An examination of the scree plot also indicated one factor. All items loaded saliently $(\mathrm{L}>.87)$ on one and only one factor. In addition, the threefactor CFA of the WDI inventory sub-scales used in this study provides support for the theorized three-factor structure, which includes diversity climate as one factor. Finally, an examination of the between-level SRMR fit indices indicated that the poor overall model fit may be due to the lack of fit in the between-level structure. Thus, the maximal model was run, in which the between-level portion of the CFA was saturated, so the model fit indices would reflect only the fit at the individual-level (Hox, 2002). All hypotheses in this study were made at the individual level, so examining model fit at the individuallevel follows logically in the context of this study. After conducting the maximal model multilevel CFA, the fit indices greatly improved, indicating good model fit $\left(\chi^{2}[3]=9.77\right.$, $p=.02, \operatorname{RMSEA}=.07, \mathrm{SRMR}=.01$ [within], .02 [between], $\mathrm{CFI}=1.00$, and $\mathrm{TLI}=.97)$.

Organizational justice. Organizational justice was measured using four items and it was theorized to be unidimensional. A single-level CFA was conducted to test for unidimensionality, and the fit indices suggested excellent model fit $\left(\chi^{2}[2]=.02, p=.99\right.$, $\mathrm{RMSEA}=.00, \mathrm{SRMR}=.001, \mathrm{CFI}=1.00, \mathrm{TLI}=1.01)$. In addition to the single-level analysis, a multilevel CFA was also conducted, with the fit indices also indicating excellent model fit $\left(\chi^{2}[8]=.40, p=.99, \mathrm{RMSEA}=.00, \mathrm{SRMR}=.00, \mathrm{CFI}=1.00, \mathrm{TLI}=\right.$ 1.02). 
Organizational identity. Organizational identity was measured using four items and theorized to be unidimensional. A single-level CFA was conducted to test for unidimensionality, and the fit indices suggested poor model fit $\left(\chi^{2}[2]=11.06, p<.001\right.$, RMSEA $=.10, \mathrm{SRMR}=.03, \mathrm{CFI}=.94, \mathrm{TLI}=.83$ ). Thus, a multilevel CFA was conducted for organizational identity, with the fit indices improving to indicate excellent model fit $\left(\chi^{2}[8]=8.72, p=.37, \mathrm{RMSEA}=.02, \mathrm{SRMR}=.04, \mathrm{CFI}=1.00, \mathrm{TLI}=.99\right)$.

Creative performance. For the outcome variables, both employee self-rated creative performance and supervisor-rated creative performance were theorized to be unidimensional and single-level CFAs were conducted to test this factor structure.

Employee self-rated creative performance. The alternative fit indices for employee self-rated creative performance suggested poor model fit $\left(\chi^{2}[65]=280.96, p<\right.$ $.001, \mathrm{RMSEA}=.10, \mathrm{SRMR}=.05, \mathrm{CFI}=.92, \mathrm{TLI}=.90)$. Thus, a multilevel CFA was conducted, and the fit indices improved to indicate adequate model fit $\left(\chi^{2}[143]=296.90\right.$, $p<.001, \mathrm{RMSEA}=.06, \mathrm{SRMR}=.05, \mathrm{CFI}=.94, \mathrm{TLI}=.94)$.

Supervisor-rated creative performance. The fit indices for the single-level CFA of supervisor-rated creative performance suggested acceptable model fit $\left(\chi^{2}[65]=\right.$ $242.28, p<.001, \mathrm{RMSEA}=.09, \mathrm{SRMR}=.04, \mathrm{CFI}=.94$, and TLI $=.93)$. A multilevel CFA of supervisor-rated creative performance was conducted to see if model fit would improve, but it did not $\left(\chi^{2}[143]=463.59, p<.001, \mathrm{RMSEA}=.08, \mathrm{SRMR}=.06, \mathrm{CFI}=\right.$ .88 , and $\mathrm{TLI}=.87)$.

Personality control variables. The personality control variables, proactive personality and openness to experience, were measured using more than one item, so 
CFAs were conducted to test the theorized factor structure of each. Both controls were theorized to be unidimensional, so single-level CFAs were first conducted to test model fit. The results for both indicated poor model fit $\left(\chi^{2}[35]=299.65, p<.001\right.$, RMSEA $=$ $.14, \mathrm{SRMR}=.08, \mathrm{CFI}=.78, \mathrm{TLI}=.71$ and $\chi^{2}[9]=121.63, p<.001, \mathrm{RMSEA}=.18$, SRMR $=.10, \mathrm{CFI}=.91, \mathrm{TLI}=.85$, respectively), so multilevel CFAs and further analyses were conducted for both.

Proactive personality. The multilevel CFA indicated that the model fit improved but remained questionable $\left(\chi^{2}[80]=178.68, p<.001, \mathrm{RMSEA}=.06, \mathrm{SRMR}=.06, \mathrm{CFI}\right.$ $=.92, \mathrm{TLI}=.91)$. A review of the literature on the psychometric properties of the proactive personality scale used in this study (Seibert, Crant, \& Kraimer, 1999) revealed that two recent studies used the Mandarin version of this scale with Chinese participants. Zhou and Shi (2009) conducted a study to determine the reliability and validity of the 10item scale. Using four samples, with the first two being very similar to the sample used in this study, and they found similar fit indices to the multilevel CFAs presented above $(\mathrm{RMSEA}=.07, \mathrm{CFI}=.92, \mathrm{NNFI}=.90)$. In addition, Baba, Tourigny, Wang and Liu (2009) studied proactive personality and work performance in China. In their examination of this scale, they found that only one item did not meet their criteria for inclusion, so that item was deleted (they did not indicate which item was deleted). To further examine the scale in the context of this study, the standardized factor loadings from the single-level CFA were examined and only one item was below .40 (Proactive Personality Item 9, "If I believe in an idea, no obstacle will prevent me from making it happen"). Next, the corrected item-total correlations were examined, and the same item 
noted above had a corrected item-total correlation below .40. A content review of this relatively poor-performing item revealed that it was too similar in content to a wellperforming item (Proactive Personality Item 5, "No matter what the odds, if I believe in something, I will make it happen") to delete on the basis of the statistical information alone. Thus, in light of previous research indicating support for the 10-item scale and a content review of the poor-performing item, all 10 items were retained in further analysis.

Openness to Experience. To further examine the construct validity of openness to experience, a multilevel CFA was conducted, and similar to proactive personality, the model fit improved but remained questionable $\left(\chi^{2}(48)=283.46, p<.001\right.$, RMSEA $=.11$, $\mathrm{SRMR}=.15, \mathrm{CFI}=.74, \mathrm{TLI}=.69)$. A review of the literature on the psychometric properties of the Saucier's (1994) Mini-Marker items used to measure openness to experience in this study revealed no recent studies having taken place in China. However, Thompson (2009) found suboptimal psychometric properties of the sub-scale with a multi-national non-native English speaking population, and he deleted two items in the scale and changed another (i.e., imaginative to "unimaginative"). In addition, research generally supports the validity of the five-factor model (Oh et al., 2013), but some parts of the model have been found to more robust than others. The factor in question, openness to experience, is the most controversial (Hough \& Ones, 2001).

To further examine the performance of the scale in this study, a four-step process was conducted. First, the standardized factor loadings from the single-level CFA were examined, and four items were found to be below .20 (i.e., philosophical, complex, deep, unintellectual). Second, corrected item-total correlations were examined, and the same 
four items noted above had corrected item-total correlations below .40. Third, a content analysis of the items indicated that the poor-performing items may not be as relevant to the high-tech context of this sample. Fourth, an exploratory factor analysis using principle axis factoring and direct oblimin rotation was conducted, with results indicating there were two factors with eigenvalues over 1 . The first factor had an eigenvalue of 3.81 , explaining $45.51 \%$ of the variance, while the second had an eigenvalue of 1.69 , explaining $13.14 \%$ of the variance in item responses. An examination of the scree plot also indicated two factors. Finally, an examination of the structure matrix showed the well-performing items loading on one factor with loadings of .89 or higher. The poor performing items loaded onto the second factor with loadings ranging from .37 to .63 . In summary, Thompson (2009) deleted two of the same problematic items in his study (complex and unintellectual), and the factor structure of this scale has been called into question in the literature. In this study, the results of the CFA, correlation analysis, content analysis, and the EFA indicated that the same four items performed poorly in the context of this sample. Thus, it was decided to retain only the four well-performing items for further analysis (i.e., to create the composite that measured openness to experience). To ensure reliability and validity were not negatively affected by this change, Cronbach's alpha was computed and a multilevel CFA with the four retained items was conducted ( $\alpha$ $\left.=.96, \chi^{2}(8)=20.20, p<.001, \mathrm{RMSEA}=.06, \mathrm{SRMR}=.07, \mathrm{CFI}=.97, \mathrm{TLI}=.96\right)$. Thus, the openness to experience scale was modified for the purposes of this study to ensure unidimensionality of the composite used in the path analyses. 


\section{Bi-variate correlations}

Bi-variate correlations for all variables in the study were computed based on the results of the best fitting models found in the CFAs described above. They are presented in Table 8, and a number of significant correlations among the variables were found. In addition, the demographic control variables (age, gender, organizational tenure and education) were group mean centered to account for the variance due to team membership.

Main variables. Transformational leadership was significantly and positively correlated with diversity climate $(r=.40, p<.01)$, organizational justice $(r=.35, p<$ $.01)$, organizational identity, $(r=.40, p<.01)$, and employee self-rated creative performance $(r=.25, p<.01)$. It did not have a significant relationship with supervisorrated creative performance $(r=-.02, n s)$.

Diversity climate was significantly and positively correlated with organizational justice $(r=.63, p<.01)$, organizational identity $(r=.54, p<.01)$, and employee selfrated creative performance $(r=.22, p<.01)$. It also had no significant relationship to supervisor-rated creative performance $(r=.03, n s)$. Organizational justice was significantly and positively related to organizational identity $(r=.52, p<.01)$ and employee self-rated creative performance $(r=.17, p<.01)$, but not to supervisor-rated creative performance $(r=.05, n s)$. Organizational identity was significantly and positively related to employee self-rated creative performance $(r=.24, p<.01)$, but not to supervisor-rated creative performance $(r=.09, n s)$. 
Finally, employee self-rated creative performance was significantly and positively related to supervisor-rated creative performance $(r=.37, p<.01)$.

Control variables. The personality control variables had several significant relationships with the main variables. Proactive personality was positively and significantly related to all but one focal variable, including transformational leadership ( $r$ $=.11, p<.05)$, diversity climate $(r=.17, p<.01)$, organizational justice $(r=.13, p<$ $.05)$, organizational identity $(r=.19, p<.01)$, self-rated creative performance $(r=.37, p$ $<.01)$, and supervisor-rated creative performance $(r=.10, p<.05)$. Openness to experience was negatively and significantly related to transformational leadership $(r=-$ $.14, p<.01)$, but none of the other predictor variables. It was positively and significantly related to the control variable of proactive personality $(r=.36, p<.01)$.

Several significant relationships emerged among the group mean centered demographic controls and the main variables. Age was positively and significantly related to employee self-rated creative performance $(r=.15, p<.01)$. Gender was not significantly related to any of the variables except age, which was a negative relationship $(r=-.11, p<.05$, with female $=0$, male $=1)$. Organizational tenure was negatively and significantly related to transformational leadership $(r=-.16, p<.01)$ and diversity climate $(r=-.10, p<.05)$. It was also a strongly positive and significant correlate to age $(r=.60, p<.01)$. Finally, education was positively and significantly related to employee self-rated creative performance $(r=.11, p<.05)$ but no other variables in the study.

Taken together, the results of the single- and multilevel CFAs, the tests of comparative model fit, the EFAs, and bi-variate correlations provided evidence for the 
use of composites for each construct in the models analyzed in this study and described below.

\section{Descriptive statistics}

The descriptive statistics presented in Tables 7 and 8 for the control and main variables were calculated based on the results of the best fitting models found in the CFAs described above. All items were equally weighted in calculating the composite scores. An examination of the histograms of the continuous variables revealed observed normal distributions. Using descriptive statistics within SPSS 17, the values for skewness and kurtosis were computed. When examined, none were found to be above the criteria provided by West, Finch, and Curran (1995) for determining whether or not data violates the assumption of normality (i.e., none with skewness values greater than two or kurtosis values greater than seven).

Reliability analysis. In examining zero-order Cronbach's alpha for each construct measured with more than one item (Cronbach, 1955), most indicated a high degree of internal consistency (see Table 8). That is, most alpha values were above the commonly accepted minimum for research of .70 (Cortina, 1993; Schmitt, 1996). These values were calculated without taking nesting by team or organization into account due to the low values for the ICC(1)s for the variables (see Tables 9 and 10).

Organizational Justice. With all four items measuring organizational justice included in the reliability analysis, Cronbach's alpha was acceptable but not excellent ( $\alpha$ $=.72$ ). When one negatively-worded item (i.e., higher scores on the items indicated lower levels of the construct) was deleted, reliability improved substantially $(\alpha=.88)$. The 
construct validity remained strong, since with three items, it was just identified $\left(\chi^{2}[3]=\right.$ $.00, p=.99, \mathrm{RMSEA}=.00, \mathrm{SRMR}=.00, \mathrm{CFI}=1.00, \mathrm{TLI}=1.01$; see Table 4$).$ Thus, the composite of organizational justice used in the path analyses was calculated by averaging the three remaining items.

Organizational Identity. With all four items measuring organizational identity included in the analysis, Cronbach's alpha was below the acceptable level of internal consistency in research $(\alpha=.52)$. When the one negatively-worded item was deleted, reliability improved substantially $(\alpha=.60)$, but remained below the generally accepted standard. The further deletion of items caused reliability to drop below .60. Thus, the composite of organizational identity used in the path analyses was calculating by averaging the three remaining items.

Considering organizational justice and organizational identity together, the poor performance of the deleted items for each construct appears to be due to a method effect, since both were negatively-phrased.

\section{Testing for dependence}

Values of ICC (1) were calculated using Mplus version 5.21 (Muthén \& Muthén, 2010), including the following variables: transformational leadership, diversity climate, organizational justice, organizational identity, self-rated creative performance, and supervisor-rated creative performance. Table 9 lists the ICC(1) values for each variable using team as the cluster variable. From these analyses, it was concluded that employee self-rated creative performance did not vary a great deal based on supervisor (ICC $[1]=$ .03); however, since even ICC(1) values of .01 can inflate Type I error rates 
(Barcikowski, 1981), both single-level and multilevel analyses were conducted for the model using employee self-rated creative performance as the outcome variable (ECP model).

In addition, the analyses indicated that the extent of nesting (or dependence) of creative performance ratings by the 64 supervisors in the study should be accounted for in further analyses $(\operatorname{ICC}[1]=.32)$. Thus, multilevel path analysis was conducted for the model using supervisor-rated creative performance as the outcome variable (SCP model).

When examining the extent of nesting for both self- and supervisor-rated creative performance based on the three organizations from which data was gathered, no significant effects were found for either outcome variable $(\mathrm{ICC}[1]=.01$ for both self- and supervisor-rated creative performance; see Table 10). Therefore, it was concluded that the effect of organization need not be accounted for in further analyses.

\section{Hypothesis testing}

An overview of the hypotheses testing results are presented in Table 11. Table 12 presents the chi-square values, degrees of freedom, and model fit indices for the full models. Results of the path analyses for the ECP model (with self-rated creative performance as the outcome variable) and the SCP model (with supervisor-rated creative performance as the outcome variable) were computed using Mplus version 5.21 (Muthén \& Muthén, 2010), and the beta weights and $p$-values for both models are presented in Figures 3-6 and Tables 13-23.

Due to concerns regarding potential multicollinearity issues, the path models were tested with one less-relevant control variable (age) deleted from the analysis. The 
rationale for deleting age from the models was that it was a highly positive and significant correlate to organizational tenure (zero-order correlation: $r=.60, \mathrm{p}<.01$ ). Since organizational tenure is a more theoretically relevant construct in creativity research, this variable was retained in the analysis and age was deleted for the hypotheses testing.

Main effects of the ECP Model. Figures 3 and 4 and Tables 13-17 present the results of the single- and multilevel path analyses for the ECP model, using employee self-rated creative performance as the endogenous outcome variable.

Single-level ECP Model. Tables 13 and 14 present the results of the single-level path analysis of ECP model (using only individual-level variables). The tables and results presented below include the standardized beta weights. As noted above, the demographic control variables were group mean centered.

Workplace Diversity Constructs. Table 13 details the beta weights and significance values for the control and focal variables with the three constructs of workplace diversity included in this study when they and the other predictors are included in the equation with self-rated creativity as the ultimate outcome variable.

Diversity climate was found to be positively and significantly related to transformational leadership $(\beta=.39, p<.001)$. The education control variable approached significance and was negatively related $(\beta=-.08, p=.08)$, as was proactive personality, with a positive relationship to diversity climate $(\beta=.09, p=.08)$.

Organizational justice was negatively and significantly predicted by openness to experience $(\beta=-.11, p=.01)$. The interaction of transformational leadership and 
diversity climate approached significance in positively predicting organizational justice $(\beta=.56, p=.08)$.

In one of the strongest relationships within the model, organizational identity was positively and significantly predicted by organizational justice $(\beta=.25, p<.001)$. Proactive personality was also a positive and significant predictor of this variable $(\beta=$ $.12, p=.01)$ and openness to experience was a negative and significant predictor $(\beta=$ $-.11, p=.01)$. In addition, organizational tenure approached significance in positively predicting organizational identity $(\beta=.07, p=.09)$.

Employee self-rated creative performance. Table 14 demonstrates that none of the main variables significantly predicted the outcome variable in the single-level ECP model, employee self-rated creative performance. However, four of the control variables were significant predictors. Similar to previous research, proactive personality positively and significantly predicted self-rated creative performance $(\beta=.38, p<.001)$, as did education and organizational tenure $(\beta=.10, p=.05$ and $\beta=.15, p<.001$, respectively). However, in contrast to previous research, openness to experience was a negative and significant predictor $(\beta=-.14, p=.01)$.

Effect Sizes. To calculate the proportional variance reduction (pseudo R-square) for the multilevel models, baseline models were compared to the alternate models (the hypothesized models) based on an examination of each significant relationship found in the following results. Baseline models were created by deleting each significant pathway (and each pathway that approached significance) one at a time. Using the results from the baseline and alternate models, the Snijders and Bosker (1999) proportion reduction in 
error formula was used to calculate pseudo R-square, comparing the alternate model to the baseline model, per the notion that alternate model is expected to reduce the amount of variance unexplained by including the pathway that explains unique variance in the outcome variable. The effect sizes for significant relationships in the ECP, SCP and exploratory models analysis are presented in Table 14.

Multilevel ECP Model. Tables 16 and 17 present the results of the multilevel path analysis for the ECP model.

Workplace Diversity Constructs. Table 16 presents the results of the multilevel ECP model analysis as they pertain to the three workplace diversity constructs.

Consistent with the single-level analysis results, transformational leadership was found to be a strongly positive and significant predictor of diversity climate $(b=.48, p<$ .001). None of the control variables significantly predicted this construct. Only education approached significance in negatively predicting diversity climate $(b=-.06, p=.08)$.

Regarding organizational justice, the interaction of transformational leadership and diversity climate approached significance $(b=.10, p=.08$; see Figure 7). One control variable, openness to experience, was a significant predictor, in the negative direction $(b=-.07, p=.01)$.

Lastly, organizational justice was found to positively and significantly predict organizational identity $(b=.15, p<.001)$. Transformational leadership and diversity climate approached significance in positively predicting organizational identity $(b=.28$, $p=.11$ and $b=.32, p=.08$, respectively). Two control variables, proactive personality and organizational tenure, positively and significantly predicted this variable $(b=.13, p<$ 
.001 and $b=.14, p<.001$, respectively). Conversely, openness to experience was a slightly negative and significant predictor of organizational identity $(b=-.05, p=.01)$. Employee self-rated creative performance (ECP). Unlike the analysis of the single-level ECP model, three of the focal variables were significant predictors of the main outcome variable (see Table 17). However, contrary to the hypothesized direction of the relationships, both transformational leadership and diversity climate negatively and significantly predicted ECP ( $b=-.43, p=.04$ and $b=-.52, p=.02$, respectively). The interaction of transformational leadership and diversity climate positively and significantly predicted the outcome variable $(b=.11, p=.01)$. In addition to the effects of the main variables, three control variables emerged as positive and significant predictors of ECP, education $(b=.05, p=.04)$, organizational tenure $(b=.00, p<.001)$ and proactive personality $(b=.36, p<.001)$.

In the between model, team-level diversity climate and the team-level interaction of transformational leadership and diversity climate, significantly predicted ECP (see Table 17). However, team-level diversity climate was a positive predictor $(b=.95, p=$ $.04)$ and the team-level interaction negatively predicted ECP $(b=-.17, p=.05)$. In addition, team-level transformational leadership approached significance in positively predicting $\mathrm{ECP}(b=.75, p=.08)$.

Main effects of SCP Model. Figure 5 and Tables 18 and 19 present the results of the multilevel path analysis for SCP model, using supervisor-rated creative performance as the endogenous outcome variable. The unstandardized beta weights are provided, along with the corresponding $p$ values. 
Workplace Diversity Constructs. As indicated in Table 18 and similar to the results of the ECP models, transformational leadership was a positive and significant predictor of diversity climate $(b=.48, p<.001)$. Proactive personality was a significant and positive predictor of diversity climate $(b=.13, p=.05)$, while education approached significance as a slightly negative predictor $(b=-.06, p=.08)$.

Organizational Justice. Consistent with the single- and multilevel results, Table 18 demonstrates that the interaction of transformational leadership and diversity climate approached significance in positively predicting organizational justice $(b=.10, p=.08)$. In addition, openness to experience was a slightly negative and significant predictor $(b=$ $-.07, p=.01)$.

Organizational Identity. The results regarding organizational identity are also presented in Table 18, indicating that organizational justice was a positive and significant predictor $(b=.15, p<.001)$. Similarly to the ECP multilevel model, transformational leadership and diversity climate approached significance in positively predicting organizational identity $(b=.28, p=.11$ and $b=.32, p=.08)$. The beta weights and $\mathrm{p}$ values for the control variables remained the same as the ECP multilevel model as well.

Supervisor-rated creative performance. The within-level analysis presented in Table 19 indicates that organizational identity was the only positive and significant predictor of SCP $(b=.16, p=.01)$. Gender negatively and significantly predicted the outcome variable $(b=-.22, p=.03$, female $=0$, male $=1)$. Organizational tenure was a positive and significant but not practically relevant predictor $(b=.00, p=.05)$. Education approached significance in positively predicting $\operatorname{SCP}(b=.05, p=.06)$. 
At the group level of analysis, none of the team-level variables significantly predicted SCP. However, the two of the centered control variables in the between model, education and proactive personality, positively and significantly predicted $\operatorname{SCP}(b=.17$, $p=.01$ and $b=.57, p<.001)$. Gender was found to approach significance in negatively predicting $\operatorname{SCP}(b=-.49, p=.07$, female $=0$, male $=1)$.

An analysis of the single-level SCP model was also conducted, and the results indicated that a few of the significant relationships (or relationships approaching significance) found in the multilevel model did not emerge in the single-level model. Thus, it is logical to conclude that accounting for variance due to the team-level variables is important.

Mediation analyses. The results of all tests of indirect effects are presented in Table 20 .

Single-level ECP model. In the single-level analysis of the ECP model, support was found for Hypothesis 1, in which it was predicted there would be a significant indirect effect of transformational leadership on creative performance through diversity climate $(\beta=.04, p=.04)$. However, contrary to the predictions in hypotheses $3,3 \mathrm{e}, 4$, and $4 \mathrm{f}$, the results of the other tests of indirect effects indicated that no other indirect effects were significant (see Table 23). In addition, the single-level analysis of the SCP model did not produce any significant indirect effects.

Multilevel ECP and SCP models. None of the indirect effects tested in the multilevel models were significant. 


\section{Exploratory analyses}

Additional tests of indirect effects. While only one of the mediation hypotheses was supported, the results of the main effects analyses suggested that it may be possible for transformational leadership and/or organizational justice to have a significant indirect effect on creative performance through organizational identity. Thus, using Mplus version 5.21 (Muthén \& Muthén, 2010), these indirect effects were tested in both the ECP and the SCP models. The results are presented in Table 20. In the multilevel SCP model, organizational justice was found to have a positive and significant indirect effect on supervisor-rated creative performance through organizational identity $(b=.02, p=$ .05). In the multilevel ECP model, the indirect effect of organizational justice on employee self-rated creative performance through organizational identity was positive and approached significance $(b=.02, p=.09)$.

ECP as a predictor of SCP. Due to the strongly positive and significant correlation of employee self-rated creative performance (ECP) and supervisor-rated creative performance (SCP; $r=.37, p<.01$ ), an exploratory analysis was conducted using Mplus, in which the measure of the former was added to the multilevel SCP path analysis model (see Tables 21-23). The unstandardized beta weights and significance scores are presented below. The model fit was excellent $\left(\chi^{2}[10]=6.66, p=.76\right.$; RMSEA $=.00 ; \mathrm{SRMR}$, within $=.001, \mathrm{SRMR}$, between $=.02 ; \mathrm{CFI}=1.00 ; \mathrm{TLI}=1.01)$.

Workplace diversity constructs. Table 21 presents the results of the multilevel exploratory SCP model as it relates to the workplace diversity constructs. 
Diversity climate. Similar to the hypothesized models, transformational leadership was a positive and significant predictor of diversity climate $(b=.48, p<.001)$. In addition, education was a slightly negative and significant predictor of diversity climate $(b=-.06, p=.04)$.

Organizational justice. Consistent with the results of the SCP model, the interaction of transformational leadership and diversity climate approached significance as a positive predictor of organizational justice $(b=.10, p=.06)$. Of the control variables, openness to experience was a negative and significant predictor $(b=-.07, p=.01)$, and education approached significance as a positive predictor of organizational justice ( $b=$ $.05, p=.11)$.

Organizational identity. Lastly, organizational justice was found to be a positive and significant predictor of organizational identity $(b=.15, p<.001)$. Transformational leadership and diversity climate approached significance in positively predicting this variable $(b=.28, p=.15, b=.32, p=.12)$. Proactive personality was found to be a positive and significant predictor $(b=.13, p=.01)$, while openness to experience was a slightly negative and significant predictor of organizational identity $(b=-.05, p=.02)$. Organizational tenure approached significance in predicting this variable $(b=.00, p=$ $.08)$.

Employee self-rated creative performance (ECP). Table 22 presents the results of the multilevel exploratory model analysis on ECP. Consistent with the multilevel ECP model, the interaction of transformational leadership and diversity climate positively and significantly predicted ECP $(b=.11, p=.01)$. Diversity climate was a negative and 
significant predictor $(b=-.52, p=.05)$, while transformational leadership approached significance in negatively predicting ECP $(b=-.42, p=.07)$. Organizational identity approached significance as a positive predictor $(b=.11, p=.08)$. Additionally, three control variables, education, organizational tenure, and proactive personality, positively and significantly predicted ECP $(b=.05, p=.01 ; b=.00, p=.00 ;$ and $b=.36, p<.001$, respectively).

Supervisor-rated creative performance (SCP). Table 23 demonstrates that organizational identity and self-rated creative performance both positively and significantly predicted SCP $(b=.12, p=.02$ and $b=.37, p<.001$, respectively). The interaction of transformational leadership and diversity climate approached significance as a slightly negative predictor of $\operatorname{SCP}(b=-.08, p=.07)$. Diversity climate also approached significance in positively predicting $\operatorname{SCP}(b=.39, p=.12)$. Gender and proactive personality, two control variables, were found to be negative and significant predictors of SCP $(b=-.21, p=.00$ and $b=-.08, p=.03$, respectively).

In the between model, two team-level predictors, transformational leadership and diversity climate significantly and negatively predicted SCP $(b=-3.27, p=.05$ and $b=$ $3.62, p=.04$, respectively). The interaction of these variables positively and significantly predicted SCP $(b=.72, p=.04)$. One control variable, proactive personality, positively and significantly predicted SCP $(b=1.06, p=.02)$. 


\section{Chapter 8: Discussion}

\section{Main Findings}

Integrating research on leadership, workplace diversity, and creativity, this study examined the direct and indirect effects of two contextual variables, transformational leadership and diversity climate, and the interaction between them, on individual creative performance through organizational justice and organizational identity. While certain parts of the major hypotheses did not receive support, the overall pattern of the results supported the argument that transformational leadership and the dimensions of workplace diversity influence individual creative performance.

Interactions. Hypothesis 2 predicted that positive employee perceptions of organizational diversity climate would interact with leader behaviors characteristic of transformational leadership to result in increased individual creative performance. This study found that the interaction of transformational leadership and diversity climate significantly predicted employee self-rated creative performance (ECP), and the interaction term approached significance in predicting supervisor-rated creativity (SCP) in the exploratory multilevel model (with ECP predicting SCP). These results have important practical and academic implications, and they are notable considering the literature that demonstrates the difficulty of finding significant interactions. The practical implication of these results is that in order to inspire higher levels of creativity among workers, it is not enough for leaders to exhibit transformational leadership behaviors, nor it is enough for employees to perceive a strong and positive diversity climate. Rather, this 
finding provides evidence for the predicted multiplicative effect - that is it important to focus efforts on both increasing transformational leadership behaviors and ensuring diversity is an explicit priority in which the organization invests in a meaningful manner. These results seem to indicate that an organization must value and promote diversity as a strategic organizational asset (develop a strong and positive diversity climate), and it must provide vision, strategic direction, and stimulation for employees to think outside the box (transformational leadership) in order to promote the creativity that is the lifeblood for many companies, especially high tech firms such as the ones in this study.

From a research perspective, this may provide an explanation for some of the mixed findings in the leadership, diversity, and creativity literatures. It may be that the mixed findings have occurred due to a lack of considering the combined effect of leadership and diversity climate. These results indicate that it may be important to include the interaction of transformational leadership and diversity climate when studying creativity.

However, while the interaction was significant in the multilevel ECP model, the components of the interaction term (transformational leadership and diversity climate) negatively and significantly predicted employee self-rated creativity. Contrary to prediction and previous research, this result indicates that the interaction of the two variables tended to result in lower self-rated creative performance (see Figure 8). However, these results are in opposition to a large body of research showing that both transformational leadership and diversity climate positively predict creativity. In this study, transformational leadership is positively and significantly related to ECP $(r=.25$, 
$p<.001)$, as is diversity climate $(r=.22, p<.001)$. Given that the main effect terms, transformational leadership and diversity climate, are highly correlated with the interaction term that is composed of these variables, the beta weights of transformational leadership and diversity climate predicting ECP and SCP are unreliable and largely uninterpretable when the cross product is included in the equation for the path analysis. That is, the high level of correlation modifies the main effects when the interaction term is entered. Thus, the results of the main effects must be interpreted in light of this multicollinearity issue. That stated, it remains that these results suggest that the self-rated measure of creative performance may operate differently in Chinese work settings and future research is necessary to replicate and/or attempt to explain these effects.

Consistently significant findings. In addition to the significant effect of the interaction term on creative performance (Hypothesis 2), this study found consistent support for Hypothesis $4 \mathrm{~d}$, that organizational identity would positively and significantly predict creative performance. The two other most consistent findings are that transformational leadership positively and significantly predicted diversity climate (Hypothesis 1a), and that organizational justice does the same for organizational identity (Hypothesis 4e).

Self-rated vs. supervisor-rated creative performance. Overall, the multilevel ECP model (using employee self-rated creativity as the outcome variable) demonstrated the strongest effects on creative performance, providing more support for the hypotheses of this study than did the multilevel SCP model (with supervisor-rated creativity as the outcome). That is, five hypothesized relationships reached significance (including three 
predicting employee self-rated creative performance) and four approached significance in the multilevel ECP model. By comparison, the multilevel SCP model results indicated three significant hypothesized relationships, including the two found in all models (transformational leadership predicting diversity climate and organizational justice predicting organizational identity), and importantly, organizational identity significantly predicting SCP.

This overall finding is in line with a recent meta-analysis which demonstrated that in most cases, the effect sizes of studies on creative performance are larger when employee self-ratings of creativity are used as compared to non-self-report measures, such as supervisor reports (Ng \& Feldman, 2012). First of all, creative performance seems to be qualitatively different from other dimensions of work performance because, until the outcome manifests, it includes a number of internal processes that are difficult for supervisors or co-workers to observe. In addition, the meta-analytic results indicate that the measures of creativity at work are also inter-related but distinct, with different patterns of results, as noted above.

A first inclination may be to attribute this to common method variance; however the longitudinal design and the time-lags among predictors and the creative outcome make this unlikely (Ng \& Feldman, 2012). There is more on this point later in the Strengths subsection of this Discussion. Ng, Feldman, and other creativity researchers also note a number of theoretical reasons to explain why self-rated creativity may be the most effective measure of creative performance. First, employees are more aware of their own creative thoughts and actions at work. The supervisor, or even other co-workers are 
not likely to know all of the creative actions of a certain employee, but that employee will know the extent and context of his/her creative work performance. Second, creativity is discretionary behavior in that it is not usually a defined part of job, so creative actions may not receive much attention from the supervisor. The focus may instead be on completing the prescribed tasks at hand, so the supervisor (or peers) are not as primed to notice creative behaviors. Third, being creative at work may involve potential risk and/or competition among co-workers, making it necessary for an employee to be strategic and possibly covert in the manner and timing with which his or her creative ideas are shared in the organization. This may be especially true in risk-avoidant cultures. In the context of this study, there is some evidence that China's national culture is risk-averse relative to other cultures around the world (House et al., 2004; Javidan et al., 2006), so this assertion may be especially pertinent here. However, this same effect has also been theorized to be true in Western settings, referred to as creative deviance (Mainemelis, 2010). Thus, the supervisor might not be aware of all the creative actions of his or her employees. It is likely due to the above-stated reasons that research has found different patterns of results for self-rated and supervisor-rated measures of creativity, with the effect sizes of studies on creative performance being larger when self-rated measures are used (Ng \& Feldman, 2012). This study provides further evidence that self- and supervisor- rated creativity have different predictors and outcomes. For example, the interaction of transformational leadership and diversity climate significantly predicted ECP, while it did not significantly predict SCP. In addition, organizational identity significantly predicted SCP, while it approached significance in predicting ECP. 
Overview of hypothesis testing results. To provide an overview of this study's findings, the significant results of the hypothesis testing are interpreted here. A hypothesis is considered supported if: (a) $p \leq .05$, and (b) the beta weight is in the expected direction. Overall, the results indicated that four of the ten main effects hypotheses were supported in the multilevel ECP or SCP model. Additionally, four hypothesized relationships approached significance $(\mathrm{p} \leq .10)$. Two other hypothesized main effects relationships were found to be significant, but in the opposite direction of prediction. The results of the main effects hypotheses are discussed below. Of the three moderation hypotheses, one was supported. Finally, of the five mediation hypotheses, one was supported, while another approached significance. In addition, of the two exploratory mediation analyses conducted, one was significant, while another approached significance. These results are discussed individually below.

The zero-order bivariate correlations, which demonstrated that transformational leadership was positively and significantly related to each dimension of the workplace diversity taxonomy and to $\mathrm{ECP}$, but not to $\mathrm{SCP}$, are similar to recent empirical findings (e.g., Pillai et al.,1999; Kark, Shamir, \& Chen, 2003; Wieland, 2004). In addition, the level of correlation between self-rated and supervisor-rated creative performance is in line with previous research $(r=.37)$. Janssen (2000) found that self-ratings of creativity were correlated with leader-ratings of creativity at .35. Because this study uses constructs mainly developed and tested in Western contexts (other than transformational leadership), that fact that the same general pattern of findings emerged in a Chinese setting is a substantial contribution to the literature. 
Hypothesis 1. The first hypothesis was broken into four parts, with the overall prediction being that transformational leadership would have a significant indirect effect on creative performance through diversity climate. Support was found for this mediational hypothesis in the single-level analysis of the ECP model.

Examining the results of the sub-parts of this hypothesis, it was found that the results of all single- and multilevel models (using both ECP and SCP as the outcome variable) supported Hypothesis 1a: transformational leadership was a positive and significant predictor of diversity climate. This finding indicates that higher levels of employee perceptions of supervisor transformational leadership tend to lead to more positive perceptions of the organization's diversity climate. However, hypotheses $1 b$ and $1 c$ were not supported in the SCP model, yet in the ECP model, the hypothesized direction of prediction was switched (i.e., from positive to negative) for transformational leadership and diversity climate each predicting self-ratings of creative performance. However, these findings are counter-balanced by the discussion above of the high correlation of these variable with the interaction term included in the equation.

Hypothesis 2. The second hypothesis, that the interaction of transformational leadership and diversity climate would positively and significantly predict creativity, was supported in the multilevel ECP model. It was proposed that the interaction would be a multiplicative, rather than an additive, effect with transformational leadership and diversity climate interacting significantly to predict creative performance, and these results were discussed above. 
Hypothesis 3. The third hypothesis was a moderated mediation hypotheses, that there would be a significant indirect effect of the interaction of transformational leadership and diversity climate on creative performance through organizational justice. This hypothesis was not supported in either model. However, Hypothesis $3 \mathrm{c}$ received partial support when tested in both the ECP and SCP models. That is, the interaction of transformational leadership and diversity climate approached significance in predicting organizational justice. Figure 7 demonstrates that employees perceive the highest levels of organizational justice when diversity climate is more strongly positive and when leaders demonstrate more transformational leadership behaviors. However, when transformational leadership is very low, employee perceptions of organizational justice tend to be higher when perceptions of diversity climate are low. This interaction suggests that it if transformational leadership is mostly lacking in an organization, employees may perceive more fairness at work when the organization does not place a high priority on diversity. This suggests that effective leadership is important in managing a diverse environment, otherwise, the priority ascribed to diversity may seem like window dressing only, rather than a substantive commitment.

Since the beta weights of the interaction and its components in predicting organizational justice were similar for both measures of creativity, only one figure is presented and interpreted because the results and interpretation are the same.

However, the other sub-sections of Hypothesis 3 were not supported. The lack of significant findings regarding organizational justice in this study is discussed in a subsequent section. 
Hypothesis 4. The fourth hypothesis was not supported in either model; however, a few of the sub-sections were supported. In all single- and multilevel ECP and SCP models, organizational justice was a positive and significant predictor of organizational identity (Hypothesis 4e). In addition, 4d predicted that organizational identity would be a positive predictor of creative performance, and this hypothesis was supported in the SCP model. In the ECP model, this relationship approached significance. The results of Hypotheses $4 \mathrm{a}$ (transformational leadership predicting organizational identity) and $4 \mathrm{~b}$ (diversity climate as a predictor of organizational identity) indicated that both relationships approached significance in the ECP and SCP models.

Pseudo R-square values. While the pattern of prediction slightly differed between self-rated and supervisor-rated creativity, the significant predictors in the model accounted for similar amounts of variance. In both the ECP and SCP models, the pathway in which transformational leadership predicting diversity climate accounted for the highest amount of variance, at $14 \%$ (pseudo R-square $=.14$ ). The relationship with the second-highest effect size in both the ECP and SCP models was organizational justice predicting organizational identity (pseudo R-square $=.04$ ). In the ECP model, the pathway in which the interaction of transformational leadership and diversity climate predicted ECP accounted for $2 \%$ of the variance (pseudo R-square $=.02$ ). In the SCP model, it was the relationship of organizational identity predicting SCP that accounted for the third-highest amount of variance (pseudo R-square $=.02$ ). For both models, the other significant relationships—or those that approached significance—accounted for $1 \%$ of the variance or less. 
Single-level analysis vs. multilevel analysis of the ECP model. The single-level path analysis that tested the ECP model demonstrated similar but fewer significant results than the multilevel model with the same outcome. Specifically, in both models, transformational leadership was a positive and significant predictor of diversity climate and organizational justice was a positive and significant predictor of organizational identity. Overall, the single-level ECP model resulted in two significant focal relationships, and one approaching significance, while the multilevel ECP model resulted in five significant focal relationships and four that approached significance.

Demographic control variables. Because the ICC(1) values indicated a certain level of nesting by team, the demographic control variables (age, gender, education, organizational tenure) were group mean centered to separate the impact of any team-level effects from the individual-level variables. That is, the team mean of each control variable was subtracted from each individual score. In the context of the multilevel modeling conducted in this study, partitioning the individual-level effects from the teamlevel effects in the demographic control variables is important so that the individual effect of each control variable can be isolated and can account for its own portion of the variance, without the team-level effects distorting the results. Similarly, in modeling the team-level effects, the same demographic controls were group mean centered to account for the team-level effects separated out of the individual level variables. This is the standard frog pond model discussed by Bliese and Jex (2002). For example, by centering the control variable of organizational tenure, the individual-level effects of one's own tenure on creative performance can be estimated, without the impact of the rest of the 
team's organizational tenure diluting or distorting the results of this individual-level analysis. If the team-level effects were not partitioned, it may be that one group has a higher mean tenure than another group, and it may be the average team tenure that accounts for differences in creative performance, rather than one's own individual tenure. Separating the individual-level variance from that of the team provides a clearer and more accurate account of the control variables' impact on the focal constructs of this study.

Several of the control variables were found to significantly predict the focal variables in the study. However, providing further evidence to the assertion above that ECP and SCP have different antecedents, the way in which the control variables predicted ECP and SCP differed. Of particular note were the results regarding gender. In the multilevel SCP model, it was found in both the within- and between-level analysis that gender was a negative and significant predictor of supervisor-rated creative performance. With males coded as " 0 " and women coded as " 1 ", this finding in the within-analysis indicates that controlling for the gender composition of the team, supervisors in this sample tended to rate men higher in creative performance than women. In the between model, this finding indicates that controlling for an individual's gender, supervisors in this sample tended to provide higher ratings of creative performance for teams with a higher composition of men than women. That is, the more men on one's team, regardless of one's own gender, the more likely supervisor ratings of creative performance would be higher than an individual's ratings on a team composed mostly of women. In the ECP model, gender was not a significant control variable. 
Three control variables positively and significantly predicted ECP: proactive personality, education, and organizational tenure. Only organizational tenure positively predicted SCP, but similar to the ECP model, the beta weight was less than .01. In addition, education approached significance $(p=.06)$ in positively predicting SCP.

\section{Exploratory Analyses}

Exploratory mediation analyses. The results of the main effects hypotheses provided evidence that there may be a significant indirect effect of transformational leadership on creative performance through organizational identity, and or organizational justice on creative performance through organizational identity. The exploratory mediation analyses were conducted in both the ECP and SCP models. The findings indicated that in the multilevel SCP model, there was a significant indirect effect of organizational justice on creative performance through organizational identity. Similarly, in the multilevel ECP model, the results approached significance. The fact that the model using the supervisor-rated measure of creative performance was significant, while the self-rated measure only approached significance, demonstrates the strength of this effect, since SCP was the outcome. As noted above, meta-analytic research has shown that effects tend to be stronger when using self-reported measures of creativity ( $\mathrm{Ng} \&$ Feldman, 2012).

These results indicated that while organizational justice was not a significant predictor of SCP, it does have an impact on creativity through organizational identity. That is, if an employee perceived a high level of fairness at work (i.e., organizational justice), it was more likely that he or she identified with one's co-workers (i.e., had a high 
level of organizational identity). This in turn tended to result in higher supervisor ratings of creative performance. Thus, this study provides evidence that organizational identity is a mechanism through which the organizational justice-creative performance relationship occurs in the particular Chinese work contexts of this study.

Chinese culture has been described as having comparatively high national averages on in-group collectivism (House et al., 2004; Hofstede, 2001; Taras et al., 2010). Due to the likelihood of a tendency to promote group harmony above an individual sense of fairness or justice, it follows that organizational identity would be a driving force for creative performance. Instead of being primarily motivated by perceived fairness in the workplace to improve performance, which has been found in North American and European contexts (Clark \& James, 1999; James, in press), this study suggests that the perception of inclusion in one's work team partially explains why perceptions of fairness would lead to creativity at work.

Exploratory model analysis: ECP predicting SCP. The results of this model were similar to the hypothesized multilevel SCP model discussed above, with a few major differences: first, self-rated creative performance was a positive and significant predictor of supervisor-rated creative performance. This indicates that an employee's selfevaluation of creative performance tends to positively impact the way in which a supervisor rates the employee on creativity. This adds support to recent findings that an employee's own evaluation of his/her ability to be creative (similar to the concept of creative self-efficacy) results in higher supervisor ratings of creative performance (Tierney \& Farmer, 2002). A potential explanation for this finding is that as an employee 
becomes more aware of his or her own creative actions at work, he or she will determine ways to let others know about the new and useful ideas or processes developed. Especially if the organization values employees being creative at work, this effect seems likely. However, further investigation of the relationship between self-rated and supervisor-rated creativity should occur to substantiate this claim and to determine the mechanisms of the relationship.

\section{Potential explanations for unexpected findings}

While the general pattern of results supported the argument that transformational leadership and the dimensions of workplace diversity influence individual creative performance, four interesting exceptions to this pattern of results emerged.

Low reliability of the organizational identity construct. The Taxonomy of Workplace Diversity construct of organizational identity had low reliability $(\alpha=.60)$, which demonstrates that further research on the taxonomy should include a focus on examining and developing this construct. It also indicates that this construct may be operationalized differently in Chinese settings. However, given the low level of reliability, it is surprising that the results showed that it significantly predicted SCP, and approached significance in predicting ECP. In addition, the positive and significant prediction of this construct by organizational justice was one of the most robust findings of this study. These results provide evidence for the strength of these effects (i.e., that significance was found, even with the low level of reliability of the organizational identity construct). 
Significant mediation found at single-level only. While the multilevel ECP model generally produced more significant results than single-level ECP model, the only significant hypothesized mediation was found in the single-level ECP model. Specifically, support was found for Hypothesis 1, which predicted there would be a significant indirect effect of transformational leadership on creative performance through diversity climate. Since the multilevel model is a more conservative estimate of the beta weights and significance scores, this finding indicates that team differences explain some of the variability that is contributing to the significant single-level indirect effects.

\section{Nonsignificance of transformational leadership predicting supervisor-rated}

creative performance. One possible explanation for the lack of finding a significant relationship between transformational leadership and SCP is that the level at which the former is measured may be stifling the predicted effect. It may be that the behaviors of higher level leaders, rather than the employee's direct supervisor, tends to impact employees' creative performance. In this study, transformational leadership was measured at the direct supervisor level only, while previous studies including multi-level models used measures of executive-level transformational leadership as well. These studies have found that measures of higher-level organizational leaders' transformational leadership produced stronger effects of creative performance or the mechanisms that led to increased creativity. Specifically, James and Lahti (2011) found that supervisory charismatic leadership had a statistically positive, but weaker, effect than executive charismatic leadership on employee vision inspiration, which was found to be a mediator of individual creativity. Thus, it may be that executive- level, or higher-level, 
organizational leaders provide the vision, charisma, and inspiration that motivate employees to be creative. To be consistent with previous research regarding the impact of transformational leadership at higher levels on creative performance, future studies should include measures of this construct at higher levels of management than an employee's direct supervisor.

\section{Transformational leadership negatively predicting self-rated creative}

performance. In this study, higher levels of transformational leadership negatively and significantly predicted self-rated creativity (ECP). One explanation is the inclusion of the interactional term in the model, which pulls variance from the main effect due to the very high correlation between the interaction term and its components. Another potential explanation can be found in the critiques of the way in which transformational leadership is operationalized in different cultures. While there are numerous empirical findings that point to the utility of transformational leadership across cultures (Singer \& Singer, 1990; Bass, 1997; Den Hartog et al., 1999; Javidan et al., 2006) and to the effectiveness of transformational leadership in Chinese contexts in particular (Aryee, Walumbwa, Zhou, \& Hartnell, 2012; Si \& Wei, 2012; Zhu, Newman, Miao, \& Hooke, 2013), there may be some credence to the argument that behaviors expressing certain dimensions of transformational leadership may vary across cultures (Barling et al., 2011). Gertsner and Day (1994) found evidence that different cultures value different leader traits. Ah Chong and Thomas (1997) demonstrated that, even within the same national culture (i.e., New Zealand), preferences for leadership varied as a function of leader and follower ethnicity. 
More research on the way in which transformational leadership is operationalized should be conducted in China.

\section{Diversity climate negatively predicting self-rated creative performance.}

Regarding the unexpected negative direction of the relationship between diversity climate and ECP, one explanation can be found in the discussion of the interaction term in the previous section. However, this construct required extra attention in the preliminary analyses to find evidence of unidimensionality. A potential explanation for this may be drawn from the current political situation in China. Although there are 56 ethnic groups in China, the Han ethnic group comprises the vast majority of China's population at $91 \%$ of 1.3 billion people (Ohio State, 2013). While each of the rest of the 55 minority ethnic groups may be small in number, they exert a powerful force on the political climate in China. Examples of the influence of these minority groups include the global Free Tibet initiative, which has protesters at nearly every high-profile global event in which China is involved, and the Uighur rebellion. Due to this political climate, it may be that Chinese citizens, and especially Chinese employees, are sensitized to issues of diversity. This awareness of social group differences may affect the way in which Chinese employees respond to questions about diversity climate. It is also reasonable to suggest that the more an organization focuses on diversity, the more sensitive the employees may be to social and group differences. This sensitivity may increase caution in the work place, so as not to offend anyone at work or get in trouble with management, which may in turn impact how employees answer survey items regarding diversity. 
Another potential explanation for the initial issues with construct validity is that diversity climate may be conceptually unclear in a Chinese context. While the terms used in the diversity climate items of the WDI (e.g., diversity initiatives) are salient, relevant, and relatively easily understood in most U.S. settings, this may not be the case in China. A literature search revealed no articles on diversity climate in a Chinese context, so it is difficult to ascertain whether or not this is the case. However, even in U.S. contexts, the lack of a clear consensus on the definition of workplace diversity (as discussed in chapter 3) makes the construct difficult to operationalize. The WDI is an attempt to operationalize workplace diversity by delineating the multiple dimensions of this complex construct. Taylor, James, and colleagues (2012) have found initial evidence of construct validity. Diversity climate is one of the dimensions of the WDI, so it follows that the construct may be defined and measured differently in Chinese contexts.

Future research should include qualitative and quantitative examinations of diversity climate in Chinese settings. Such research could include focus groups asking participants what comes to mind when the term "diversity" is used in a work context. In the U.S., many organizations have instituted diversity initiatives at one level or another, so U.S. participants are likely to have some indication of what the term "diversity" refers to in a work context. Thus, they would be able to understand and consistently respond to the items in the diversity climate scale (e.g., "my organization puts a lot of resources into diversity initiatives"), as initial evidence of the WDI suggests (Taylor, Murry, \& James, 2012). This may or may not be the case among Chinese participants. Future research should also investigate whether or not Chinese workers have similar or different 
associations with the term "workplace diversity" and respond similarly (in structure rather than content) to the items in the WDI diversity climate scale. Such investigations may help researchers develop a more culturally appropriate measure of diversity climate that will demonstrate higher structural validity and a potentially different pattern of relationships. Scholars have called for more empirical attention to the climates or cultures that facilitate the positive effects of diversity on work outcomes (Guillaume, Dawson, Woods, Sacramento, \& West, 2013), especially outside Western contexts.

\section{Potential explanations for nonsignificant results}

It is prudent to discuss the organizational justice construct included in the study, since only one of the hypotheses that included organizational justice was supported in both models. Meta-analytic research has demonstrated there are three empirically distinct dimensions of organizational justice (Colquitt et al., 2001; Cohen-Charash \& Spector, Paul, 2002). The organizational justice subscale of the WDI is unidimensional, and the findings of this study suggest that perhaps the measure should be expanded to include all three factors.

While the measure may need further development, China's cultural profile may also help explain the lack of significant findings for this variable. As noted above, China ranks highly on in-group collectivism. In a culture that highly values in-group harmony, fairness at work may not motivate employees in China as it has been found to do so in U.S. contexts and other national contexts with lower average scores on collectivism. In addition, China has a stronger orientation towards hierarchy (Javidan et al., 2006) and a relatively higher score on the cultural dimension of power distance than North American 
and Western European countries (House et al., 2004), so the perceived fairness of leader actions may not be as much of a concern to employees in China. It may be that the concern lies more in maintaining group harmony and relationships among co-workers and the leader or supervisor. However, future research in Chinese work settings using well-designed qualitative and quantitative research methods, with a focus on understanding the cultural context, should examine these assertions more fully.

\section{Taxonomy of Workplace Diversity}

Regarding the relationships among the nomological network of workplace diversity, three of the seven dimensions of the taxonomy were included in the model, and as expected, the bivariate correlations indicated that these dimensions were significantly and positively related at the predicted levels. Diversity climate was significantly and positively related to organizational justice $(r=.63, \mathrm{p}<.01)$ and organizational identity $(r=.54, \mathrm{p}<.01)$, and organizational justice was significantly and positively related to organizational identity $(r=.52, \mathrm{p}<.01)$. While these variables were positively and significantly related, they were not related at such high levels that they would be considered to be the same construct. This provides further evidence of convergent validity for these dimensions of the workplace diversity taxonomy, adding to that developed by Taylor and colleagues (2011). Additionally, the only other variables in the study to correlate as highly with each other were the two control variables of age and organizational tenure, $r=.60, p<.001$ ), which was in line with theoretical expectations due to the conceptual associations of these constructs. This high level of association also provided part of the rationale for excluding age from the pathway analysis. 
The findings in this study provide support for separately examining the different dimensions of workplace diversity in research and practice, rather than including diversity as one unidimensional variable. Because the development of the nomological network of workplace diversity and the instrument developed to measure it (i.e., the Workplace Diversity Inventory) is in its nascent stages, examining the relationships among the dimensions within it extends our knowledge of this conceptual model of workplace diversity.

\section{Strengths}

Embedded within the design of this study are several methodological strengths. First, the design is longitudinal; data for the focal variables were collected at three different time points. This multi-wave data helps to counteract the limitations of common method variance, which may be a concern due to the fact that all data were collected via employee surveys (Podsakoff et al., 2003). Unlike cross-sectional designs, this study can inform assertions regarding direction of causality. It also responds to the need for more rigorous research designs in the field of creativity (Zhou \& Shalley, 2011) and diversity (Jackson \& Joshi, 2011).

Second, as a field study conducted with employee and supervisor participants, the study has the potential to be more generalizable (i.e., higher external validity) than experimental studies conducted in the lab (Zhou \& Shalley, 2011) or field studies conducted among university students. This likelihood is increased because the design is not cross-sectional, as most field studies on creativity have been (Ng \& Feldman, 2012; Zhou \& Shalley, 2011). 
A third strength of the study is that it was conducted using a non-Western sample, increasing the representativeness and generalizability of the research on the topics of transformational leadership, diversity, and creativity.

\section{Implications for Research}

The findings of the single- and multilevel models have several implications for the study of the three broad focal constructs (leadership, diversity, creativity). First, they provide further evidence for the validity of the nomological network of workplace diversity, which provides an operational definition of this complex construct by examining and determining its antecedents, correlates, and outcomes. By including three dimensions of workplace diversity in the study, the differential impacts of each dimension have been parceled out and examined individually. Transformational leadership was included in the study as an antecedent to three dimensions of workplace diversity: diversity climate, organizational justice, and organizational identity. Including creative performance as an outcome variable in this study has provided further insight into the nomological networks of both creativity and workplace diversity regarding the following relationships: 1) creativity as an outcome of the interaction of transformational leadership and diversity climate, 2) creativity as an outcome of organizational identity, 3) transformational leadership as a predictor of diversity climate, 4) organizational justice as an antecedent of organizational identity, 5) diversity climate as an antecedent of organizational identity, and 6) the interaction of transformational leadership and diversity climate predicting organizational justice. Adding to the body of literature regarding the 
above relationships in a Chinese context is a valuable contribution to three bodies of literature.

Second, the findings provide insight into the distal and proximal variables that predict creative performance, which is important to scholars to explain and predict the contextual and individual-level factors that enhance creativity. This will help researchers develop evidence-based recommendations for organizational leaders to anticipate and respond to changes in today's fast-paced work environments.

Of the main effects hypotheses with less empirical support listed in the introduction, two were supported and two hypothesized relationships approached significance in this study, including the following:

Full Support:

1a. Transformational leadership will be significantly and positively related to perceived organizational diversity climate.

2. Transformational leadership and diversity climate will interact to significantly affect individual creative performance (supervisor-rated).

Approached Significance in ECP and SCP Models:

4a. Transformational leadership will be significantly and positively related to perceived organizational identity.

4b. Diversity climate will be significantly and positively related to perceived organizational identity.

Finally, this study begins to answer the call from Osland, Taylor, and Mendenhall (2009) to integrate global leadership and traditional leadership theories and research.

Since the findings were somewhat similar to those theorized and found in Western contexts, especially regarding the direction of the significant relationship between 
transformational leadership and diversity climate, as well as that of organizational justice and organizational identity, the results provide evidence that behaviors characteristic of transformational leadership may help Chinese leaders effectively handle the increased complexities and ambiguities of the globalized economy. This assertion is discussed more below.

\section{Practical Implications}

There are several practical implications that are relevant for all types of organizations. First, the findings inform practitioners of the important leader behaviors and diversity dynamics that may help enhance employee creative performance. After decades of research on workplace diversity and numerous meta-analytic investigations, scholars still know very little about the necessary conditions and the mechanisms by which diversity affects individual, team, and organizational outcomes (Avery \& McKay, 2010). There is also a disappointing lack of insight into which leader behaviors are most effective to leverage the benefits of a diverse workforce (Guillaume et al., 2013). The findings of this study contribute to filling this gap in the literature by providing additional insight into the behaviors and dynamics that can help organizational leaders manifest the positive outcomes of diversity - the most relevant and important of these outcomes being increased employee creativity.

In the midst of increasingly diverse workforces, both domestically and around the world, the results of this study highlight the importance of developing a strong organizational identity among employees so they feel included, perceive they are part of the team, and identify with the organization. The findings of this study suggest that 
organizations can help increase their employee's organizational identity by training leaders to demonstrate transformational leadership behaviors (especially inspirational motivation and intellectual stimulation) and by doing everything possible so that employees perceive fairness in how procedures are implemented and how people are treated (i.e., increasing organizational justice). The current study provides evidence that increasing organizational justice tends to led to increased organizational identity. When this occurs, the findings of this current study suggest that employees will be more willing and able to share their different perspectives and experiences in order to develop innovative solutions and/or products that respond to the rapidly changing demands, problems, and opportunities of the globalized economy. This study suggests that fostering a strong sense of organizational identity is a principal motivator of employee creative performance in a Chinese work setting and that it mediates the organizational justicecreativity relationship.

This study also demonstrates the importance of specifying the dimensions of diversity which organizational leaders should consider in attempting to increase employee creativity. The findings reinforce the common advice of practitioners to specify which dynamics of diversity are of most concern in specific organizational or work group contexts.

In addition, the results of this study provide evidence that leader behaviors are important determinants of whether or not an organization will reap the benefits—or fall prey to the potential pitfalls — of workplace diversity. Organizations should intentionally and strategically train leaders to exhibit behaviors characteristic of transformational 
leadership and build a positive diversity climate. Specifically, leaders should be trained to develop and communicate the organization's vision and values and to set over-arching strategic goals from which employees can develop cascading team and individual goals that align with the organizational vision. In addition, to build a positive diversity climate, organizational leaders should demonstrate they value diversity by connecting the mission and strategic direction of the organization to diversity. Such actions are especially important for new employees during the on-boarding process (Bauer, Bodner, Erdogan, Truxillo, \& Tucker, 2007). To support this assertion, research has demonstrated that good socialization processes enhance newcomers' adjustment, which in turn leads to improved organizational performance, employee job satisfaction, and organizational commitment, as well as a decrease in turnover intentions and actual turnover (Bauer et al., 2007).

\section{Limitations and Future Research}

There are several limitations to the study that suggest the need for future research. The first limitation involves the use of self-report measures for all variables except the outcome variable. This is in spite of the fact that the design is longitudinal and that the dependent variable was measured using supervisor ratings (as well as employee selfreport ratings). Second, all of the data was collected via one method (i.e., employee surveys), so common method bias may be a limiting factor of the study. These two limitations potentially threaten the validity of the results regarding the relationships among the constructs (Podsakoff et al., 2003) because the regression coefficients may be inflated due to using the same method to collect the data and the same source (as in the 
case of the ECP model). However, the longitudinal design of the study counteracts the majority of this concern.

A third limitation is the restricted applicability of the model to predict only individual creative performance, rather than overall task performance or another dimension of performance (e.g., organizational citizenship behaviors, proactive performance). Some scholars have chosen to focus on task performance (Kearney \& Gebert, 2009), stating that creative performance is too narrow a focus when looking at the broad constructs of transformational leadership and workplace diversity. However, this limitation does not substantially decrease the usefulness of the current findings, since increased creativity is one of the most commonly touted benefits of diversity (Jackson \& Joshi, 2011). In addition, future research on team-level creative performance would be important in future refining and testing of the proposed model.

A fourth limitation is that not all dimensions of transformational leadership were measured. While this was an intentional part of the research design, examining the way in which all dimensions of transformational leadership impact the relationships in the tested models would deepen our knowledge of these constructs and the way in which they are related. Future research should focus especially on the dimension of individualized consideration in Chinese workplaces, due to the proposed conceptual link between transformational leadership and global leadership (explained further below, i.e., that individualized consideration would likely be an effective tool for global leaders to use to influence the work behaviors of many different types of people). 
A final limitation is that the current model does not cover all types and dimensions of diversity. However, the ability of one study to do so is highly unlikely. In addition, Jackson and Joshi (2011) illustrated the importance of explicitly stating which types of diversity are being examined within each study, and Taylor and colleagues (2012) emphasized the criticality of examining specific dimensions of diversity, rather than conceptualizing and/or operationalizing diversity as a one-dimensional construct. The proposed study answers both of these calls within the literature. It would be extremely difficult to include and address all types and dimensions of workplace diversity in a single study. Thus, examining the different relationships among the types and dimensions of diversity, as well as their associations to critical organizational outcomes, is fertile ground for future research.

While evidence was found for the significant effect of the interaction of transformational leadership and diversity climate on employee self-rated creative performance, the discrepancy between the results of the self-rated and supervisor-rated creativity measures leave many questions to be answered. Future research should examine these relationships further and in different contexts. In addition, other possible moderators and mediators of the relationships among leadership, diversity, and creativity should be examined. The mediational pathways of organizational identity should be studied further, since the exploratory analyses in the study revealed a significant indirect effect of organizational justice on creative performance through organizational identity.

Regarding workplace diversity, meta-analytic evidence regarding main effect approaches have proven to be of little use to explain the effects of diversity on work 
outcomes (Bell, 2007; Bowers, Pharmer, \& Salas, 2000; Guillaume et al., 2012; Horwitz \& Horwitz, 2007; Joshi \& Roh, 2009; Stewart, 2006, van Dijk, van Engen, \& van Knippenberg, 2012; Webber \& Donahue, 2001; Wood, 1987). Thus, scholars have called for the examination of the underlying psychological processes influencing work behaviors in diverse work settings (Avery \& McKay, 2010). The lack of examining these processes has been a major limitation of diversity research (McKay, Avery, \& Morris, 2008). A recent study found that one such psychological process, i.e., psychological safety, mediated the relationship between diversity climate and employee performance (Singh, Winkel, \& Selvarajan, 2013). The variables in the nomological network of workplace diversity should also be further examined as potential moderators and mediators of the relationships between leadership and creative performance.

\section{Transformational Leadership and Global Leadership}

In the context of this study, the conceptual link between transformational leadership and global leadership is essential to consider and discuss. In Chinese organizations generally, and in Chinese high-tech companies (the focal organizations of this study) particularly, there is a need to be competitive on a global level in terms of creativity and innovation in technical product development. For Chinese workers to generate ideas for products and technology that appeal to and have traction with consumers around the world, they need to understand something of the mentality of the people in other cultures and demonstrate an active interest in continually learning more. Thus, for Chinese companies to be competitive in today's fast-paced global economy, they need to promote a global outlook in their organizational visions. To do so, it is 
necessary for leaders of Chinese organizations to articulate to their employees the importance of understanding market demands around the world. Providing the purpose underlying the focus on external markets would help employees espouse the vision and be inspired by it, resulting in the increased likelihood that the products they develop are competitive in the global marketplace. By promoting an outward focus and encouraging creativity to meet the demands of global markets, organizational leaders can help their companies grow and prosper.

Providing a clear vision and strategic direction for employees to work toward is conceptually related to the inspirational motivation dimension of transformational leadership, i.e., providing a vision and rationale for employees to be creative and establishing goals toward creative performance. In addition to the need for Chinese companies to articulate a vision, promote a global outlook, and set overarching goals for creativity, developing and cultivating a positive organizational diversity climate is likely to nurture, to some extent, a globalized mentality.

However, scholars have noted the tendency for Chinese managers to have a negative view of leaders who have a global outlook (Javidan et al., 2006; House et al., 2004). This may be explained in part by China's relatively high score on in-group collectivism, which indicates Chinese employees may view the world outside China as an out-group, and as a result, tend to be less interested in anything outside their in-group (Javidan et al., 2006). Thus, in Chinese contexts, successful transformational leadership includes valuing diversity and counteracting the potential inclination of Chinese employees to view non-Chinese foci as less important. As a result, effective leaders in 
this context would need to make intentional efforts to endorse a global outlook in the organization and to enumerate the advantages of doing so to employees, especially in terms of innovative product development and global competition. This global outlook, often called global mindset, is a defining element of global leadership (Osland et al., 2009; Levy et al., 2007). It is defined as being composed of two constructs: cognitive complexity and cosmopolitanism. Cognitive complexity is being able to differentiate and integrate diverse ideas and perspectives, and cosmopolitanism is having an enthusiastic interest and appreciation of other cultures (Osland et al., 2009; Levy et al., 2007). Being proficient in these two elements would help Chinese leaders provide the necessary vision and strategic direction discussed above.

Given the imperative discussed above for Chinese managers to have a global mindset and to promote a global outlook among employees, it is argued here that global leadership is an important construct to examine in the context of this study due to its conceptual connection to transformational leadership. This leads to a discussion of global leadership to explain its association to the research and application of transformational leadership. The conceptual link asserted here is that the competencies characteristic of transformational leadership will support the global leadership that is required of Chinese leaders today. If a Chinese manager is skilled in transformational leadership behaviors, he or she is more likely to be successful in promoting the global outlook that is essential for high-tech firms — such as those participating in this study—striving to be successful in today's increasingly competitive and complex globalized economy. 
Global leadership. Due to the rapid pace of globalization, many companies need to develop and carefully implement global strategies that provide access to new markets and supply chains. Accordingly, there is a great need for competent global leaders to execute these strategies. However, Osland, Bird, Mendenhall, and Osland (2006) cite a distinct lack of skilled global leaders, which is due in part to the fact that being a global leader is very challenging. Global leaders must navigate the ambiguities and complexities of the global business environment, implement new and constantly changing strategies, and leverage the opportunities globalization creates for companies (Beechler \& Javidan, 2007), such as encouraging and utilizing employee creativity and innovation.

Global leadership was introduced relatively recently as a construct for academic study. The term "global leader" first appeared in the 1960's to describe an organization's place in the market, and it was only in the 1980's that it was applied to individuals (McCall \& Hollenbeck, 2002, pp. 20-21). However, much of this work has focused on expatriates, rather than on global leaders specifically. Most of the current published work on the topic offers practical, normative advice to global executives and human resource professionals, rather than addressing the theoretical or empirical questions surrounding the concept (Osland et al., 2006). The empirical research on global leadership developed and first took root in the organizational behavior and management literature, and it has received attention in Industrial/ Organizational Psychology (see Holt \& Seki, 2012).

The construct of global leadership is defined as the process of influencing the thinking, attitudes and behaviors of a global organization to work together synergistically toward a common vision and common goals (Osland, Taylor \& 
Mendenhall, 2009). Global leadership can be exercised by an individual or a group.

Global leaders must rely on non-traditional and varying levels of hierarchical means of influence to be effective with a variety of people from different backgrounds and cultures.

One of the primary ways in which global leadership has been theoretically distinguished from within-nation (i.e., domestic) leadership is that global leaders must adapt to the demands of significantly greater complexity. Bird and Osland (2004) outlined the following demands with which a global leader must contend:

1) a heightened need for cultural understanding within a setting characterized by wide-ranging diversity; 2) greater need for broad knowledge that spans functions and nations; 3 ) wider and more frequent boundary spanning both within and across organizational and national boundaries; 4) more stakeholders to understand and consider when making decisions; 5) a more challenging and expanded list of competing tensions both on and off the job; 6) heightened ambiguity surrounding decisions and related outcomes/effects; 7) more challenging ethical dilemmas relating to globalization. Simply put, the transition from purely domestic to global is a quantum leap (p. 61).

While the field of global leadership is relatively new and there is not yet a solid consensus on the parameters of the global leadership construct and how to measure it (Osland et al., 2006), a broad theoretical foundation has been developed and new research is expanding and deepening it. Further empirical testing is needed using global participants and settings, and scholars have also called for the integration of global leadership and Western, domestic leadership theories (Osland, Taylor \& Mendenhall, 2009). 
In an attempt to respond to this call, a discussion is provided here of the way in which global leadership research and transformational leadership research may be integrated. In recent years, research on transformational leadership has expanded into the area of global and cross-cultural leadership. While the research has been somewhat mixed, there is convincing evidence that transformational leadership is effective across cultural and national borders (Aryee, Walumbwa, Zhou, \& Hartnell, 2012; Bass, 1997; Chhokar, Brodbeck, \& House, 2007; Den Hartog et al., 1999; House et al., 2004; Javidan et al., 2006; Si \& Wei, 2012; Singer \& Singer, 1990; Zhu, Newman, Miao, \& Hooke, 2013). The numerous translations of the Multi-factor Leadership Questionnaire (MLQ; Felfe, 2006; Shao \& Webber, 2006) are another indicator of the cross-national popularity and utility of transformational leadership. Specifically, Den Hartog and colleagues (1999) hypothesized and found evidence to support the assertion that "several attributes associated with transformational leadership are universally seen as contributing to outstanding leadership" (p. 242) in the GLOBE research program. Thus, due to its demonstrated effectiveness across cultures and contexts, it appears that the behaviors of transformational leadership would help a leader inspire and motivate his or her direct reports to follow the organization's vision and strategic direction.

Thus, the assertion is made here that transformational leadership may help individuals effectively manage the increased complexity and uncertainty brought on by rapidly increasing globalization — the factor spurring the need for global leadership. In other words, developing the competencies of transformational leadership is likely to help global leaders deal with the increased demands of the global marketplace. In this way, the 
present study describes how the two bodies of research on transformational leadership and global leadership may be integrated. To further explain the relationship between the concepts, a review of the GLOBE study and a brief discussion of how the results relate to transformational leadership will illuminate how this assertion is supported by current research.

GLOBE. The GLOBE research program was a ten-year study conducted in 62 societies designed to conceptualize, operationalize, test, and validate a cross-level theory of the relationships between culture and societal, organizational, and leadership effectiveness. Starting in 1994, a team of 170 researchers collected quantitative data from more than 17,000 middle managers in 951 organizations from three industries (i.e., financial services, food processing, and telecommunications) and gathered archival measures of a country's economic prosperity, as well as measures of the physical and psychological well-being of the cultures studied (Chhokar, Brodbeck, \& House, 2007; House, Hanges, Javidan, Dorfman, \& Gupta, 2004).

The theoretical foundation of the study is implicit leadership theory (ILT), which posits that an individual holds a set of beliefs (also referred to as prototypes, cognitive categories, mental models, schemas, and stereotypes) about the kinds of personality characteristics, skills, and behaviors that contribute to or impede effective leadership (Eden \& Leviatan, 1975). ILT predicts that this set of beliefs affects the extent to which an individual accepts and responds to others as leaders. The GLOBE researchers extended ILT to the cultural level of analysis by arguing that these belief systems (i.e., its structure and content) are shared among individuals from the same culture. The extension 
of ILT to include the culturally shared mental models was termed culturally endorsed implicit leadership theory (CLT; Den Hartog et al., 1999). GLOBE found evidence that people within cultural groups generally agree in their beliefs about leadership and that these beliefs are represented by a set of CLT profiles developed for each national culture and cluster of cultures (House at al., 2004).

In the quantitative portion of the study, the researchers conducted surveys with 112 behavioral and attribute descriptors (e.g., "honest", "informed") hypothesized to facilitate or impede effective leadership. Each descriptor included a short phrase to help participants interpret the item. Participants rated the items on a 7-point scale $(1=$ this behavior or characteristic greatly inhibits a person from being an outstanding leader; $7=$ this behavior or characteristic contributes greatly to a person being an outstanding leader).

In one part of the mammoth study, the research team empirically categorized the 112 leadership descriptors into six dimensions (i.e., the CLT profiles) and determined which leadership attributes were universally effective, and which were culturally contingent. The criteria for being categorized as a universally effective leadership attribute were the following: "(1) $95 \%$ of country scores had to exceed a mean of 5 on a 7-point scale for that item/attribute; and (2) the grand mean score for all countries had to exceed 6 for the item/attribute" (Den Hartog et al., 1999, p. 237). One of the six CLT profiles—charismatic/value-based leadership—is conceptually similar to transformational leadership, and the following three sub-dimensions of this CLT were found to be endorsed as effective leadership attributes across all cultures: 1) integrity, 
including items describing leaders as trustworthy, just, and honest; 2) visionary, including items regarding foresight and planning ahead; and 3) inspirational, including items such as being positive, encouraging, dynamic, motivational, and confidence building. As stated above, Den Hartog and colleagues (1999) concluded that these universally endorsed characteristics reflected the dimensions of transformational leadership. To extend these findings to the context of the current study, it is asserted here that these subdimensions are conceptually similar and roughly map onto three of the four dimensions of transformational leadership, namely, charisma/idealized influence (integrity and inspirational), inspirational motivation (visionary), and individualized consideration (inspirational). Thus, results of the massive GLOBE research program suggest that transformational leadership is conceptually similar to leadership attributes that have been endorsed as effective across cultures and nations (House et al., 2004), providing support for the assertion that proficiency in transformational leadership competencies may increase global leaders' effectiveness.

Given that transformational leadership can be desirable and effective across cultures generally, making its development attractive to leaders across the globe, it is further argued here that transformational leadership competencies will support the global leadership required of Chinese leaders today. How transformational leadership may help increase global leader effectiveness requires close examination.

First, the transformational leadership dimension of inspirational motivation and its linkage to global leadership is examined for two reasons: 1) it is conceptually similar to the global leadership competency of vision (Bird \& Osland, 2004), and 2) it may help 
leaders balance global consistency and local responsiveness. As stated above, inspirational motivation is defined as the development and articulation of a compelling vision and the establishment of difficult but realistic goals to move toward that vision (Bass, 1985). In their 2006 review of the global leadership literature, Osland, Bird, Mendenhall, and Osland categorized 53 competencies for global leaders into six dimensions, one of which is visioning. The competencies listed under this dimension were the following: "articulates a tangible vision and strategy, articulates values, (acts as a catalyst for culture change, (acts as a) catalyst for strategic change" (p. 209). The first two competencies listed by Osland and colleagues (i.e., articulates a tangible vision and strategy, articulates values) align almost exactly with the definition of inspirational motivation, showing that these two aspects of transformational leadership and global leadership are conceptually related. The second reason to consider inspirational motivation is the likelihood that demonstrating these behaviors (i.e., communicating a compelling vision and setting difficult and specific goals) can help a leader act in a "glocal" manner (i.e., acting in a way that takes both global and local concerns into account; Begley \& Boyd, 2003). That is, it may help global leaders provide the overall vision and values necessary to create a globally consistent culture (i.e., global), while at the same time ensuring the practices of subsidiaries are responsive to the national culture in which they are embedded (i.e., local; Begley \& Boyd, 2003). When conflicting pressures arise from the global and local environments, providing a clear, high-level vision can help employees operating in subsidiaries around the world develop goals that respond to the needs of their local culture, while at the same time remain in alignment 
with the greater vision of the global organization. Demonstrating inspirational motivation can thus help global leaders create the necessary and delicate balance between providing a globally consistent organizational culture and being responsive to the different needs of local offices operating in different cultures throughout the world.

Second, it is asserted that the intellectual stimulation dimension of transformational leadership may support development of global leadership competencies. It is argued here that intellectual stimulation provides a way to operationalize one of the "threshold traits" of global leadership, i.e., inquisitiveness (Bird \& Osland, 2004). Intellectual stimulation is defined as challenging and encouraging employees to think critically, be creative, and think outside the box (Bass, 1985). Bird and Osland (2004) identify inquisitiveness as one of four threshold traits in their framework for global leadership. While the delineation of this trait was mostly in regards to the concern for global leaders to stay abreast of their global context, leaders that demonstrate intellectual stimulation are likely to do so. That is, leaders who demonstrate behaviors that motivate their employees to think critically and anticipate changes in their industry's landscape are likely to do the same themselves. In this way, they are demonstrating the type of inquisitiveness that is important for global leaders.

Demonstrating behaviors characteristic of intellectual stimulation provides a concrete way for global leaders to exhibit the personality trait of inquisitiveness. Even if a global leader is low on this theoretically stable trait, he or she can exhibit the behaviors of intellectual stimulation to help counteract the lack of a natural tendency for it. Behavior modeling training research suggests that leading by example and providing an 
opportunity to demonstrate the desired behavior is helpful in bringing about the desired outcome (Taylor et al., 2005), in this case, inquisitiveness leading to creative performance. In addition, creativity research has demonstrated that employees are more likely to be creative when they perceive that the organization values creative work (Farmer, Tierney \& Kung-McIntyre, 2003) and their supervisor encourages creative performance (Ford, 1996). Thus, by exhibiting inquisitive behaviors and encouraging employees to do the same (i.e., intellectual stimulation), global leaders are more likely to succeed in influencing employees to be creative, which can be helpful for Chinese managers in many global high tech companies.

Furthermore, intellectual stimulation is conceptually linked to one of the two major components of global mindset, i.e., cognitive complexity. Scholars have reached a consensus that having a global mindset is a defining element of global leadership (Lane, Maznevski, Mendenhall, \& McNett, 2004; Levy, Beechler, Taylor, \& Boyacigiller, 2007). As noted above, global mindset is composed of cosmopolitanism and cognitive complexity (Levy et al., 2007; Osland et al., 2006; Beechler \& Javidan, 2007). Cognitive complexity is defined as the ability to see and understand multiple perspectives and to consider ideas, people and situations from a variety of angles (differentiation) and find the connections among them (integration; Levy et al., 2007). It is argued here that in order for a leader to effectively challenge and encourage employees to think critically and be creative (i.e., demonstrate intellectual stimulation), he or she must employ a certain level of cognitive complexity. For example, it requires cognitive resources to accurately determine which situations are better suited to encourage creativity among employees 
and which situations are better for encouraging strong performance on well-defined work tasks. Thus, when a leader displays intellectual stimulation behaviors, he or she seems to be using cognitive complexity to determine when, how, to whom to inspire creativity and out-of-the-box thinking and action.

To tap the rich sources of information in today's diverse workforces, global leaders must help employees make connections between seemingly unrelated topics and knowledge. To do so, cognitive complexity enables the leader to understand the topic at hand from a variety of perspectives and to integrate these viewpoints according to the inter-connections among them. By role modeling cognitive complexity, which is asserted here to be similar to the behaviors characteristic of intellectual stimulation, global leaders may be able to help employees see numerous angles to any given situation and make the connections necessary to develop more novel and useful (i.e., creative) solutions to organizational issues and ideas for product development that resonate with global consumers and respond to a common but unrecognized need in the marketplace. In this way, the intellectual stimulation dimension of transformational leadership is linked to the concept of global leadership. In turn, this study provides evidence that the behaviors of transformational leadership (and, by association, global leadership) may increase Chinese managers' ability to inspire employees to be more creative at work.

While charisma/idealized influence dimension of transformational leadership was not included in this study, it is logical to consider it here in the context of making the connection to global leadership. Charisma/idealized influence refer to leader behaviors that provide a role model for ethical behavior and inspire employees to act in the best 
interest of the organization (Judge \& Piccolo, 2004). Behaviors characteristic of this dimension include communicating in a powerful, confident, and dynamic way, taking a public stand to do what is right in a difficult situation, and appealing to employees on an emotional level to take action that may be more difficult but is better for the team and/or company. Considering the list of six core global leadership competencies developed by Osland and colleagues (2006), the "Traits and Values" and "Visioning" dimensions contain a number of competencies that would enable a leader to exhibit charisma/ idealized influence. For example, the Visioning dimension includes the competency of articulating values and the Traits and Values dimension includes acting with integrity, both of which would help a leader to take a public stand that is aligned with his/her articulated values, which is one common behavior of exhibit charisma/ idealized influence. Adding to the discussion above about organizations needing to think "glocally" (i.e., thinking both globally and locally; Begley \& Boyd, 2003) when conflicting global and local pressures arise, a leader should be able to rely on the organizational values he or she has previously articulated and publicized. By articulating one's values, a leader provides guidance on how employees shall live out the vision and goals of the organization, especially when decisions need to be made in conflict. In this way, instead of the bottom line be the ultimate driver, the leader clearly communicates that it also matters how the goal is achieved - not just that it was achieved. This is demonstrating charisma/idealized influence. In addition, the Traits and Values dimension includes the competencies of being optimistic, energetic, and having emotional intelligence. These competencies would be useful for a leader to inspire others and communicate in a 
motivating and dynamic manner, as well as other common behaviors of this transformational leadership dimension. Charisma/idealized influence seems to be highly relevant to at least two of the six core competencies of global leadership, as listed by Osland and colleagues (2006).

The other dimension of transformational leadership not included in this study is individual consideration, and this construct is also similar to certain aspects of global leadership. Individual consideration is defined as the extent to which a leader attends to their employee's unique development needs and aspirational goals (Bass, 1985). By this definition, it is conceptually similar to two interpersonal global leadership competencies identified by Bird and Osland (2004), mindful communication and creating and building trust with individuals and groups. It seems likely that these essential global leadership competencies would help a leader demonstrate behaviors characteristic of individual consideration.

The theorized associations between the dimensions of transformational leadership and those of global leadership add to the argument presented here delineating the connections between the overall constructs of transformational leadership and global leadership. Future research is necessary to empirically examine these assertions, as well as to clarify the associations and distinctions between transformational leadership and global leadership.

In summary, transformational leadership behaviors may help global leaders more effectively navigate the increased complexities and ambiguities they face due to the global nature of their position by supporting the development of competencies deemed 
important by the global leadership literature. That is, transformational leadership seems to provide practical ways (i.e., behaviors) for global leaders to exhibit some essential global leadership competencies. If future research continues to support the conceptual connections between transformational leadership and global leadership outlined here, there are two major practical implications. First, global organizations should select leaders who have demonstrated transformational leadership behaviors in past positions. Second, leaders of global organizations should be trained in the dimensions of transformational leadership in order to enhance the global leadership competencies noted above. Transformational leadership, with its effectiveness having been demonstrated in many studies throughout the world, as well as in a recent qualitative review and metaanalysis (DeRue et al. (2011), seems to be a stepping stone toward helping to build the essential competencies of global leaders.

Given the connections between transformational leadership and global leadership argued for here this study suggests there are a few behaviors upon which the managers in this Chinese sample can focus to become more effective global leaders.

First, focusing on developing a sense of inclusion and identity with the team and organization (i.e., organizational identity) can help increase employee creativity, which as discussed above, is important for managers in Chinese high-tech firms. In addition, since the prediction of diversity climate by transformational leadership was so strongly positive and significant, it seems likely that working to increase transformational leadership behaviors would help lead to a more positive diversity climate, which has been linked to many positive organizational outcomes, not the least of which is increased creativity. 


\section{Conclusion}

This study examines the complex relationships among transformational leadership, workplace diversity and creative performance. By examining these relationships in Chinese work settings, our understanding of these constructs is deepened. The findings of this study contribute to the literature on creative performance, especially by examining the interaction of transformational leadership and diversity climate on both employee self-rated creative performance and supervisor-rated creative performance. The significant interaction of these contextual constructs on supervisor-rated creativity is a substantial contribution to the literature, since this relationship had only previously been theorized.

This study also contributes to the workplace diversity literature by lending credence to the importance of using the nomological network of diversity at work to parcel out each dimension of this multi-dimensional concept in designing workplace diversity research. It also contributes to the transformational leadership literature by adding to the growing body of literature that addresses the boundary conditions of the effects of transformational leadership.

This study specifically extends our knowledge of the associations between transformational leadership and diversity climate, organizational justice and organizational identity, and each of these variables (including the interaction of transformational leadership and diversity climate) on creative performance. The findings of this study provide insight into the mechanisms through which transformational leadership and organizational justice promote creative performance in Chinese work 
settings, i.e., through diversity climate and organizational identity, respectively. This study makes important theoretical contributions given the popularity of these constructs in the current organizational psychology and management literature. This is also true regarding the assertion that the behaviors of transformational leadership may serve as helpful tools for global leaders to navigate the increased complexity and ambiguity of leading a global organization.

Moreover, the study provides further evidence of the validity and utility of the workplace diversity taxonomy as a way to specify and isolate the vital dimensions of diversity that manifest in specific employment contexts. Its parsimonious yet thorough coverage of the domain of workplace diversity may enable scholars to account for the effects of specific dimensions of workplace diversity on important organizational outcomes, such as creative performance, individual-, team-, and organization-level performance, retention, and job satisfaction and engagement.

Future research should take into account both individual differences and contextual factors in understanding and predicting creative behaviors. Further investigation of the distal and proximal variables and processes that shape human behaviors for creativity will enhance our knowledge of this important work outcome. Increasing our understanding in this area will help scholars develop practical, data-driven advice and interventions for organizational leaders to promote and manage an essential element of performance at work - human ingenuity. 
Table 1. Typology of Work Team Diversity

\begin{tabular}{|c|c|c|}
\hline & $\begin{array}{l}\text { Diversity on relationship- } \\
\text { oriented attributes }\end{array}$ & $\begin{array}{l}\text { Diversity on task- } \\
\text { oriented attributes }\end{array}$ \\
\hline $\begin{array}{l}\text { Diversity on readily } \\
\text { detected attributes }\end{array}$ & $\begin{array}{l}\text { Gender } \\
\text { Age } \\
\text { Ethnicity } \\
\text { Nationality } \\
\text { Religion }\end{array}$ & $\begin{array}{l}\text { Department/unit } \\
\text { membership } \\
\text { Organizational tenure } \\
\text { Formal credentials and } \\
\text { titles } \\
\text { Education level } \\
\text { Memberships in } \\
\text { professional } \\
\text { organizations }\end{array}$ \\
\hline $\begin{array}{l}\text { Diversity on underlying } \\
\text { attributes }\end{array}$ & $\begin{array}{l}\text { Personality } \\
\text { Attitudes } \\
\text { Values } \\
\text { Racial/ethnic identity } \\
\text { Sexual identity } \\
\text { Other social identities }\end{array}$ & $\begin{array}{l}\text { Task knowledge } \\
\text { Organizational } \\
\quad \text { knowledge } \\
\text { Experience } \\
\text { Cognitive abilities } \\
\text { Communication skills } \\
\text { Mental models }\end{array}$ \\
\hline
\end{tabular}


Table 2. Overview of Measures by Source and Data Collection Timing

\begin{tabular}{lcc}
\hline Measure & \# Items & $\begin{array}{c}\text { Data Collection } \\
\text { Timing }\end{array}$ \\
\hline Demographics & & Time 1 \\
Proactive Personality & 10 & Time 1 \\
Openness to Experience & $8^{1}$ & Time 1 \\
Transformational Leadership & 10 & Time 1 \\
Diversity Climate & 5 & Time 2 \\
Organizational Justice & $4^{2}$ & Time 2 \\
Organizational Identity & $4^{2}$ & Time 2 \\
Employee Self-rated Creative Performance & 13 & Time 3 \\
Supervisor-rated Creative Performance & 13 & Time 3 \\
\hline
\end{tabular}

Note. ${ }^{1}$ In the employee survey, eight items were used to measure openness to experience, but four items were deleted when creating the composite score used in the path analyses due to poor item performance. ${ }^{2}$ In the employee survey, four items were used to measure these constructs, but one item was deleted when creating the composite score for each construct used in the path analyses due to poor item performance. For both constructs, the deleted item was negatively worded (i.e., higher score on the item indicated lower levels of the construct), indicating a method effect. 
Table 3. Results of Single-level Confirmatory Factor Analyses for Main and Control Variables

\begin{tabular}{|c|c|c|c|c|c|c|c|c|}
\hline Variable & $\begin{array}{l}\# \\
\text { Items }\end{array}$ & $\overline{\chi^{2}}$ & $\overline{d f}$ & $\chi^{2 / d f}$ & RMSEA & $\overline{\text { SRMR }}$ & CFI & $\overline{\text { TLI }}$ \\
\hline $\begin{array}{l}\text { Transformational } \\
\text { Leadership }^{1}\end{array}$ & 10 & 187.64 & 33 & 5.69 & .11 & .04 & .94 & .92 \\
\hline $\begin{array}{l}\text { Diversity } \\
\text { Climate }\end{array}$ & 5 & 122.70 & 5 & 24.54 & .24 & .04 & .93 & .86 \\
\hline $\begin{array}{l}\text { Organizational } \\
\text { Justice }\end{array}$ & 4 & $.02^{2}$ & 2 & .01 & .00 & .001 & 1.00 & 1.01 \\
\hline $\begin{array}{l}\text { Organizational } \\
\text { Identity }\end{array}$ & 4 & 11.06 & 2 & 5.53 & .10 & .03 & .94 & .83 \\
\hline $\begin{array}{l}\text { Employee Self- } \\
\text { Rated CP }\end{array}$ & 13 & 280.96 & 65 & 4.32 & .10 & .05 & .92 & .90 \\
\hline $\begin{array}{l}\text { Supervisor-rated } \\
\text { CP }\end{array}$ & 13 & 242.28 & 65 & 3.72 & .09 & .04 & .94 & .93 \\
\hline $\begin{array}{l}\text { Proactive } \\
\text { Personality }\end{array}$ & 10 & 299.65 & 35 & 8.56 & .14 & .08 & .78 & .71 \\
\hline $\begin{array}{l}\text { Openness to } \\
\text { Experience }\end{array}$ & 8 & 121.63 & 9 & 13.51 & .18 & .10 & .91 & .85 \\
\hline $\begin{array}{l}\text { Openness to } \\
\text { Experience }\end{array}$ & 4 & 8.00 & 2 & 4.00 & .09 & .01 & 1.00 & .98 \\
\hline
\end{tabular}

Note. ${ }^{1}$ Second-order factor model, with inspirational motivation and intellectual stimulation as first-order factors of transformational leadership; ${ }^{2} p=.99 . d f=$ degrees of freedom. $\chi^{2} / d f=$ chi square ratio. $\mathrm{CP}=$ creative performance. All $\chi^{2}$ values were significant at the $p<.01$ level unless otherwise noted. 
Table 4. Results of Multilevel Confirmatory Factor Analyses for Main and Control Variables

\begin{tabular}{|c|c|c|c|c|c|c|c|c|}
\hline Variable & $\begin{array}{c}\# \\
\text { Items }\end{array}$ & $\overline{\chi^{2}}$ & $d f$ & $\overline{\chi^{2} / d f}$ & RMSEA & $\begin{array}{c}\text { SRMR } \\
\text { (Within) }\end{array}$ & CFI & TLI \\
\hline $\begin{array}{l}\text { Diversity } \\
\text { Climate }\end{array}$ & 5 & 126.12 & 15 & 8.41 & .13 & .05 & .94 & .91 \\
\hline $\begin{array}{l}\text { Diversity } \\
\text { Climate }^{1}\end{array}$ & 5 & $9.77^{2}$ & 3 & 3.26 & .07 & .01 & 1.00 & .97 \\
\hline $\begin{array}{l}\text { Organizational } \\
\text { Justice }\end{array}$ & 4 & $.40^{3}$ & 8 & .05 & .00 & .00 & 1.00 & $\begin{array}{c}1.0 \\
2\end{array}$ \\
\hline $\begin{array}{l}\text { Organizational } \\
\text { Justice }\end{array}$ & 3 & .00 & 3 & 0 & .00 & .00 & 1.00 & $\begin{array}{c}1.0 \\
1\end{array}$ \\
\hline $\begin{array}{l}\text { Organizational } \\
\text { Identity }\end{array}$ & 4 & $8.72^{4}$ & 8 & 1.09 & .02 & .04 & 1.00 & .99 \\
\hline $\begin{array}{l}\text { Organizational } \\
\text { Identity }\end{array}$ & 3 & .23 & 3 & .08 & .00 & .00 & 1.00 & $\begin{array}{c}1.0 \\
4\end{array}$ \\
\hline $\begin{array}{l}\text { Employee Self- } \\
\text { Rated CP }\end{array}$ & 13 & 296.90 & 143 & 2.08 & .06 & .05 & .94 & .94 \\
\hline $\begin{array}{l}\text { Supervisor- } \\
\text { rated CP }\end{array}$ & 13 & 463.59 & 143 & 3.24 & .08 & .06 & .88 & .87 \\
\hline $\begin{array}{l}\text { Proactive } \\
\text { Personality }\end{array}$ & 10 & 178.68 & 80 & 2.23 & .06 & .06 & .92 & .91 \\
\hline $\begin{array}{l}\text { Openness to } \\
\text { Experience }\end{array}$ & 8 & 283.46 & 48 & 5.91 & .11 & .15 & .74 & .69 \\
\hline $\begin{array}{l}\text { Openness to } \\
\text { Experience }\end{array}$ & 4 & 20.20 & 8 & 2.53 & .06 & .07 & .97 & .96 \\
\hline
\end{tabular}

Note. ${ }^{1}$ maximal model results. ${ }^{2} p=.02 .{ }^{3} p=.99 .{ }^{4} p=.37 . d f=$ degrees of freedom. $\chi^{2} / d f=$ chi square ratio. $\mathrm{CP}=$ creative performance. All $\chi^{2}$ values were significant at the $p$ $<.01$ level unless otherwise noted. 
Table 5. CFA Results of Comparative Models for Transformational Leadership

\begin{tabular}{lccccccc}
\hline CFA Models & $d f$ & $\chi^{2}$ & $\chi^{2} / d f$ & RMSEA & SRMR & CFI & TLI \\
\hline Model 1 & 77 & $1,053.98$ & 13.69 & .17 & .10 & .72 & .67 \\
Model 2 & 76 & 440.05 & 5.79 & .11 & .06 & .90 & .87 \\
Model 3 & 74 & 252.41 & 3.41 & .08 & .05 & .95 & .94 \\
Model 4 & 72 & 252.41 & 3.51 & .08 & .05 & .95 & .93 \\
\hline
\end{tabular}

Note. $n=418 . d f=$ degrees of freedom. $\chi^{2} / d f=$ chi square ratio. In Model 1, the items of transformational leadership and organizational justice loaded on one factor. In Model 2, the transformational leadership items are loaded on one factor and the organizational justice items are loaded on another factor. In Model 3, the items for inspirational motivation, intellectual stimulation, and organizational justice are loaded onto once factor each. In Model 4, the items for intellectual stimulation and inspirational motivation are modeled as second-order factors of transformational leadership and the organizational justice items are loaded onto its own factor. Model 3 and Model 4 best fit the data among the four measurement models tested. 
Table 6. CFA Results of Comparative Models for the Workplace Diversity Inventory

\begin{tabular}{lccccccc}
\hline CFA Models & $d f$ & $\chi^{2}$ & $\chi^{2} / d f$ & RMSEA & SRMR & CFI & TLI \\
\hline $\begin{array}{l}\text { 1-Factor Model: } \\
\text { Single-level }\end{array}$ & 44 & 637.04 & 14.48 & .18 & .08 & .80 & .75 \\
$\begin{array}{l}\text { 1-Factor Model: } \\
\text { Multi-level }\end{array}$ & 99 & 651.96 & 6.59 & .12 & $\begin{array}{c}.09 \\
\text { (within) }\end{array}$ & .81 & .79 \\
$\begin{array}{l}\text { 3-Factor Model: } \\
\text { Single-level }\end{array}$ & 41 & 218.12 & 5.32 & .10 & .04 & .94 & .92 \\
$\begin{array}{l}\text { 3-Factor Model: } \\
\text { Multi-level }\end{array}$ & 137 & 295.11 & 2.15 & .05 & $\begin{array}{c}.05 \\
\text { (within) }\end{array}$ & .95 & .94 \\
\hline
\end{tabular}

Note. $n=418 . d f=$ degrees of freedom. $\chi^{2} / d f=$ chi square ratio. In the 1-factor model, all items of the Workplace Diversity Inventory scale were loaded on one factor. In 3-factor model, the diversity climate items were loaded on one factor, the organizational justice items were loaded a second factor, and the organizational identity items were loaded onto a third factor. The multi-level CFA for the 3-factor model best fit the data between the two measurement models tested. The models were tested using the items included in the employee survey (i.e., 5 items for diversity climate, and 4 items each for organizational justice and organizational identity). 
Table 7. Means and Standard Deviations of Demographic Variables

\begin{tabular}{|c|c|c|c|}
\hline Demographic Variable & Mean (years) & Standard Deviation & Range (years) \\
\hline Age & 29.37 & 5.04 & $22-59$ \\
\hline Education ${ }^{1}$ & 4.59 & 1.77 & $0-17$ \\
\hline Organizational Tenure & 3.72 & 3.91 & $.08-36$ \\
\hline Gender $^{2}$ & 0.24 & .43 & $\mathrm{n} / \mathrm{a}$ \\
\hline
\end{tabular}

Note. ${ }^{1}$ Education was measured by asking how many years of education participants had after college. 2 Gender was measured on a 2 -point scale $(0=$ male; $1=$ female $)$. 
Table 8. Means, Standard Deviations, Reliability Estimates and Correlations for Main and Control Variables

\begin{tabular}{|c|c|c|c|c|c|c|c|c|c|c|c|c|c|c|}
\hline Variable & Mean & $\mathrm{sd}$ & 1 & 2 & 3 & 4 & 5 & 6 & 7 & 8 & 9 & 10 & 11 & 12 \\
\hline 1. $\mathrm{TL}^{1}$ & 5.34 & .82 & .92 & $.51 * *$ & $.72 * *$ & $.80 * *$ & $.60 * *$ & .06 & $-.24 * *$ & $-.33 * *$ & $.20 * *$ & $.25 * *$ & $.36^{* *}$ & $.20 * *$ \\
\hline 2. $\mathrm{DC}^{2}$ & 4.95 & .95 & $.40 * *$ & .93 & $.50 * *$ & $.52 * *$ & $.45 * *$ & $.22 * *$ & .04 & $.25 * *$ & $.11 *$ & $.19 * *$ & .03 & $.17 * *$ \\
\hline 3. $\mathrm{OJ}^{2}$ & 4.95 & 1.17 & $.35 * *$ & $.63 * *$ & .88 & $.61 * *$ & $.50 * *$ & $.23 * *$ & $-.60 * *$ & $-.52 * *$ & $.33 * *$ & $-.13 * *$ & $.34 * *$ & $.43 * *$ \\
\hline 4. $I^{2}$ & 5.37 & .74 & $.40 * *$ & $.54 * *$ & $.52 * *$ & .60 & $.29 * *$ & $-.29 * *$ & $-.28 * *$ & $-.14 * *$ & $.42 * *$ & $.29 * *$ & $.55^{* *}$ & $.18 * *$ \\
\hline 5. $\mathrm{ECP}^{3}$ & 5.34 & .69 & $.25 * *$ & $.22 * *$ & $.17 * *$ & $.24 * *$ & .93 & $.67 * *$ & -.10 & $-.29 * *$ & $.38 * *$ & $.23 * *$ & $.26 * *$ & $.14 * *$ \\
\hline 6. $\mathrm{SCP}^{4}$ & 4.91 & .77 & -.02 & .03 & .05 & .09 & $.37 * *$ & .94 & .07 & -.10 & .07 & $-.17 * *$ & .01 & $.31 * *$ \\
\hline 7. $\mathrm{PP}^{5}$ & 5.27 & .77 & $.11 *$ & $.17 * *$ & $.13^{*}$ & $.19 * *$ & $.37 * *$ & $.11 *$ & .82 & $.88 * *$ & $-.30 * *$ & $-.20 * *$ & $-.61 * *$ & $-.66 * *$ \\
\hline 8. $\mathrm{OE}^{6}$ & 4.18 & 1.40 & $-.14 * *$ & .05 & -.07 & -.10 & -.01 & -.03 & $.36^{* *}$ & .96 & $-.26 * *$ & $-.25 * *$ & $-.56 * *$ & $-.60 * *$ \\
\hline 9. $\mathrm{AG}^{7}$ & .00 & 4.21. & -.09 & .00 & .03 & .05 & $.15^{* *}$ & .06 & -.01 & .01 & $\mathbf{n} / \mathbf{a}$ & $.71 * *$ & $.87 * *$ & $.43 * *$ \\
\hline 10. $\mathrm{GR}^{7}$ & .00 & .36 & -.07 & -.09 & -.04 & -.04 & -.04 & -.09 & -.05 & .04 & $-.11 *$ & $\mathbf{n} / \mathbf{a}$ & $.54 * *$ & $.33 * *$ \\
\hline 11. $\mathrm{OT}^{8}$ & .00 & 3.18 & $-.16^{* *}$ & $-.10 *$ & -.07 & -.02 & .10 & .05 & -.03 & -.00 & $.60 * *$ & .07 & $\mathbf{n} / \mathbf{a}$ & $.69 * *$ \\
\hline 12. $\mathrm{ED}^{9}$ & .00 & 1.40 & .01 & -.06 & .00 & .02 & $.11^{*}$ & .06 & .07 & .04 & .05 & -.05 & -.09 & $\mathbf{n} / \mathbf{a}$ \\
\hline
\end{tabular}

Note. $\mathrm{TL}=$ transformational leadership. $\mathrm{DC}=$ diversity climate. $\mathrm{OJ}=$ organizational justice. $\mathrm{ID}=$ organizational identity. $\mathrm{ECP}=$ employee self-rated creative performance. $\mathrm{SCP}=$ supervisor-rated creative performance. $\mathrm{PP}=$ proactive personality. $\mathrm{OE}=$ openness to experience. $\mathrm{AG}=$ age. $\mathrm{GR}=$ gender. $\mathrm{OT}=$ organizational tenure. $\mathrm{ED}=$ education. ${ }^{1} \mathrm{n}=418$, measured in wave $1 .{ }^{2} \mathrm{n}=415$, measured in wave 2 ; one measured item was deleted from the composite used in the path analysis to increase reliability and validity of constructs - organizational justice and organizational identity were measured with three items each. ${ }^{3} \mathrm{n}=356$, measured in wave $3 .{ }^{4} \mathrm{n}=318$, measured in wave $3 .{ }^{5} \mathrm{n}=371$, measured in wave $1 .{ }^{6} \mathrm{n}=371$, measured in wave 1 ; four measured items were deleted from the composite used in the path analysis to increase reliability and validity of the construct - openness to experience was measured using four items. ${ }^{7} \mathrm{n}=418$, measured in wave $1 .{ }^{8} \mathrm{n}=418$, measured in wave 1 ; organizational tenure was measured in months, converted to years in the table. ${ }^{9} \mathrm{n}=418$; education was measured using years after college. Coefficient alpha estimates are listed in bold on the diagonal. Pooled, within correlations are listed above the diagonal. All demographic control variables (age, gender, organizational tenure and education) were group mean centered. ${ }^{* *}=$ correlation is significant at the .01 level $(2$-tailed); $*=$ correlation is significant at the .05 level (2-tailed). 
Table 9. Level of Nesting in Focal Variables by Team

\begin{tabular}{ll}
\hline Variable & ICC(1) \\
\hline Transformational Leadership & .13 \\
Diversity Climate & .05 \\
Organizational Justice & .03 \\
Organizational Identity & .03 \\
Employee Self-rated Creative Performance (ECP) & .03 \\
Supervisor-rated Creative Performance (SCP) & .32 \\
\hline
\end{tabular}

Note. $n=418$. Cluster variable $=$ team. 
Table 10. Level of Nesting in Focal Variables by Organization

\begin{tabular}{ll}
\hline Variable & ICC(1) \\
\hline Transformational Leadership & .02 \\
Diversity Climate & .01 \\
Organizational Justice & .00 \\
Organizational Identity & .01 \\
Employee Self-rated Creative Performance (ECP) & .01 \\
Supervisor-rated Creative Performance (SCP) & .01 \\
\hline
\end{tabular}

Note. $n=418$. Cluster variable $=$ organization . 
Table 11. Hypothesis Tests Results

\begin{tabular}{|c|c|c|}
\hline Hypothesis & ECP Model & $\begin{array}{l}\text { SCP } \\
\text { Model }\end{array}$ \\
\hline $\begin{array}{l}\text { 1. There will be a significant indirect effect of TL on CP } \\
\text { through DC. }\end{array}$ & Support & No Support \\
\hline a. TL will be significantly and positively related to DC. & Support & Support \\
\hline b. TL will be significantly and positively related to CP. & Support $^{1}$ & No Support \\
\hline c. DC will be significantly and positively related to $\mathrm{CP}$. & Support $^{1}$ & No Support \\
\hline 2. TL and DC will interact to significantly affect CP. & Support & No Support \\
\hline $\begin{array}{l}\text { 3. There will be a significant indirect effect of TLxDC on } \\
\text { CP through OJ. }\end{array}$ & No Support & No Support \\
\hline a. TL will be significantly and positively related to OJ. & No Support & No Support \\
\hline b. DC will be significantly and positively related to OJ. & No Support & No Support \\
\hline c. TLxDC will to significantly predict OJ. & Support $^{2}$ & Support $^{2}$ \\
\hline d. OJ will be significantly and positively related to CP. & No Support & No Support \\
\hline $\begin{array}{l}\text { e. There will be a significant indirect effect of DC on } \mathrm{CP} \\
\text { through OJ. }\end{array}$ & No Support & No Support \\
\hline $\begin{array}{l}\text { 4. There will be a significant indirect effect of TLxDC on } \\
\text { CP through OI. }\end{array}$ & No Support & No Support \\
\hline a. TL will be significantly and positively related to OI. & Support $^{2}$ & Support $^{2}$ \\
\hline b. DC will be significantly and positively related to OI. & Support $^{2}$ & Support $^{2}$ \\
\hline c. TLxDC to significantly predict OI. & No Support & No Support \\
\hline d. OI will be significantly and positively $\mathrm{CP}$. & Support $^{2}$ & Support \\
\hline e. OJ will be significantly and positively related to OI. & Support & Support \\
\hline $\begin{array}{l}\text { f. There will be a significant indirect effect of DC on CP } \\
\text { through OI. }\end{array}$ & No Support & No Support \\
\hline
\end{tabular}

Note. ${ }^{1}=$ partial support, direction was opposite of hypothesized relationship. ${ }^{2}=$ approached significance. $\mathrm{TL}=$ transformational leadership. $\mathrm{DC}=$ diversity climate. TLxDC $=$ interaction term of transformational leadership and diversity climate. $\mathrm{OJ}=$ organizational justice. ID = organizational identity. $\mathrm{CP}=$ individual creative performance. 
Table 12. Model Fit Indices for ECP Model and SCP Models

\begin{tabular}{lcccc}
\hline Fit Index & \multicolumn{2}{c}{ ECP Model $^{1}$} & SCP Model & \\
\hline & Single-level & Multilevel & Multilevel & $\begin{array}{c}\text { Exploratory }_{\text {Model }^{3}} \\
\text { Multilevel }\end{array}$ \\
Chi-square & $\chi^{2}(5)=4.09$, & $\chi^{2}(10)=.6 .66$, & $\chi^{2}(10)=6.38$, & $\chi^{2}(10)=6.66$, \\
Test of & $p=.54$ & $p=.76$ & $p=.78$ & $p=.76$ \\
Model Fit & & & & \\
CFI & 1.00 & 1.00 & 1.00 & 1.00 \\
TLI & 1.00 & 1.01 & 1.01 & 1.01 \\
RMSEA & 0.00 & 0.00 & 0.00 & 0.00 \\
SRMR & 0.00 & Within: .00 & Within: .00 & Within: .00 \\
& & Between: .02 & Between: .02 & Between: 02 \\
\hline
\end{tabular}

\footnotetext{
Note. ${ }^{1}$ The ECP model used employee self-ratings of creative performance as the outcome variable; $n=418 .{ }^{2}$ The SCP model used supervisor ratings of creative performance as the outcome variable; $n=371 .{ }^{3}$ The exploratory model included employee self-ratings of creative performance predicting supervisor ratings of creative performance (SCP) and SCP was the outcome variable; $n=371$.
} 
Table 13. ECP Model: Single-level Path Analysis Results for Taxonomy of Workplace Diversity Variables

\begin{tabular}{|c|c|c|}
\hline Variable and Predictor & Beta Weight & $p$ Value \\
\hline \multicolumn{3}{|l|}{ Diversity Climate } \\
\hline \multicolumn{3}{|l|}{ Control Variables } \\
\hline Gender & -.06 & .15 \\
\hline Education & -.08 & .08 \\
\hline Organizational Tenure & -.04 & .35 \\
\hline Proactive Personality & .09 & .08 \\
\hline Openness to Experience & .07 & .17 \\
\hline \multicolumn{3}{|l|}{ Main Variables } \\
\hline Transformational Leadership & .39 & .00 \\
\hline \multicolumn{3}{|l|}{ Organizational Justice } \\
\hline \multicolumn{3}{|l|}{ Control Variables } \\
\hline Gender & .03 & .49 \\
\hline Education & .03 & .38 \\
\hline Organizational Tenure & .02 & .70 \\
\hline Proactive Personality & .06 & .15 \\
\hline Openness to Experience & -.11 & .01 \\
\hline \multicolumn{3}{|l|}{ Main Variables } \\
\hline Transformational Leadership & -.20 & .25 \\
\hline Diversity Climate & .22 & .32 \\
\hline Transformational Leadership x Diversity Climate & .56 & .08 \\
\hline \multicolumn{3}{|l|}{ Organizational Identity } \\
\hline \multicolumn{3}{|l|}{ Control Variables } \\
\hline Gender & .01 & .72 \\
\hline Education & .03 & .41 \\
\hline Organizational Tenure & .07 & .09 \\
\hline Proactive Personality & .12 & .01 \\
\hline Openness to Experience & -.11 & .01 \\
\hline \multicolumn{3}{|l|}{ Main Variables } \\
\hline Transformational Leadership & .11 & .54 \\
\hline Diversity Climate & .23 & .30 \\
\hline Transformational Leadership x Diversity Climate & .12 & .71 \\
\hline Organizational Justice & .25 & .00 \\
\hline
\end{tabular}

Note. $n=418$. 
Table 14. ECP Model: Single-level Path Analysis Results for Self-rated Creative Performance

Variable and Predictor Beta Weight $p$-Value

\section{Employee Self-rated Creative Performance}

Control Variables

Gender $-.01$

.92

Education

.10

.05

Organizational Tenure

.15

.00

Proactive Personality

.38

.00

Openness to Experience

$-.14$

.01

Main Variables

Transformational Leadership

$-.08$

.71

Diversity Climate

$-.21$

.44

Transformational Leadership x Diversity Climate

.47

.24

Organizational Justice

$-.04$

.49

Organizational Identity

.06

.31

Note. $n=418$. 
Table 15. Pseudo R-Square Values for Multi-level Models

Significant Pathway Deleted

R-Square

Estimate

\section{ECP Model}

TL predicting DC

.14

$\mathrm{OJ}$ predicting $\mathrm{OI}$

TLxDC predicting ECP

TL predicting ECP

.01

DC predicting ECP

.01

TLxDC predicting OJ

.01

DC predicting OI

.01

TL predicting OI

\section{SCP Model}

TL predicting DC

OJ predicting OI

OI predicting $\mathrm{SCP}$

.02

TLxDC predicting OJ

\section{Exploratory Model}

TL predicting DC

ECP predicting SCP

$\mathrm{OJ}$ predicting $\mathrm{OI}$

OI predicting SCP

OI predicting ECP

TLxDC predicting OJ

DC predicting OI

DC predicting ECP $\quad .00$

TL predicting ECP $\quad .00$

TLxDC predicting ECP $\quad .00$

Note. $n=418 . \mathrm{TL}=$ transformational leadership. $\mathrm{DC}=$ diversity climate. $\mathrm{TLxDC}=$ interaction of transformational leadership and diversity climate. $\mathrm{OJ}=$ organizational justice. ID = organizational identity. $\mathrm{ECP}=$ employee self-rated creative performance. $\mathrm{SCP}=$ supervisor-rated creative performance. 
Table 16. ECP Model: Multi-level Path Analysis Results for Taxonomy of Workplace Diversity Variables

\begin{tabular}{|c|c|c|}
\hline Variable and Predictor & Beta Weight & $p$ Value \\
\hline \multicolumn{3}{|l|}{ WITHIN MODEL } \\
\hline \multicolumn{3}{|l|}{ Diversity Climate } \\
\hline \multicolumn{3}{|l|}{ Control Variables } \\
\hline Gender & -.05 & .67 \\
\hline Education & -.06 & .08 \\
\hline Organizational Tenure & -.00 & .34 \\
\hline Proactive Personality & .13 & .05 \\
\hline Openness to Experience & .04 & .17 \\
\hline \multicolumn{3}{|l|}{ Main Variables } \\
\hline Transformational Leadership & .48 & .00 \\
\hline \multicolumn{3}{|l|}{ WITHIN MODEL } \\
\hline \multicolumn{3}{|l|}{ Organizational Justice } \\
\hline \multicolumn{3}{|l|}{ Control Variables } \\
\hline Gender & .11 & .43 \\
\hline Education & .05 & .19 \\
\hline Organizational Tenure & .00 & .72 \\
\hline Proactive Personality & .10 & .14 \\
\hline Openness to Experience & -.07 & .01 \\
\hline \multicolumn{3}{|l|}{ Main Variables } \\
\hline Transformational Leadership & -.32 & .25 \\
\hline Diversity Climate & .19 & .52 \\
\hline Transformational Leadership x Diversity Climate & .10 & .08 \\
\hline \multicolumn{3}{|l|}{ WITHIN MODEL } \\
\hline \multicolumn{3}{|l|}{ Organizational Identity } \\
\hline \multicolumn{3}{|l|}{ Control Variables } \\
\hline Gender & .01 & .92 \\
\hline Education & .01 & .64 \\
\hline Organizational Tenure & .00 & .06 \\
\hline Proactive Personality & .13 & .00 \\
\hline Openness to Experience & -.05 & .01 \\
\hline \multicolumn{3}{|l|}{ Main Variables } \\
\hline Transformational Leadership & .28 & .11 \\
\hline Diversity Climate & .32 & .08 \\
\hline Transformational Leadership x Diversity Climate & -.02 & .62 \\
\hline Organizational Justice & .15 & .00 \\
\hline
\end{tabular}

Note. $n=371$. 
Table 17. ECP Model: Multi-level Path Analysis Results for Employee Self-rated Creative Performance

\begin{tabular}{|c|c|c|}
\hline Variable and Predictor & Beta Weight & $p$ Value \\
\hline \multicolumn{3}{|l|}{ WITHIN MODEL } \\
\hline \multicolumn{3}{|l|}{ Employee Self-rated Creative Performance } \\
\hline \multicolumn{3}{|l|}{ Control Variables } \\
\hline Gender & -.02 & .78 \\
\hline Education & .05 & .04 \\
\hline Organizational Tenure & .00 & .00 \\
\hline Proactive Personality & .36 & .00 \\
\hline Openness to Experience & .02 & .66 \\
\hline \multicolumn{3}{|l|}{ Main Variables } \\
\hline Transformational Leadership & -.43 & .04 \\
\hline Diversity Climate & -.52 & .02 \\
\hline Transformational Leadership x Diversity Climate & .11 & .01 \\
\hline Organizational Justice & -.04 & .30 \\
\hline Organizational Identity & .11 & .06 \\
\hline \multicolumn{3}{|l|}{ BETWEEN MODEL } \\
\hline \multicolumn{3}{|l|}{ Employee Self-rated Creative Performance } \\
\hline \multicolumn{3}{|l|}{ Control Variables } \\
\hline Gender & .01 & .95 \\
\hline Education & .01 & .81 \\
\hline Organizational Tenure & .00 & .43 \\
\hline Proactive Personality & -.08 & .50 \\
\hline Openness to Experience & -.07 & .27 \\
\hline \multicolumn{3}{|l|}{ Main Variables } \\
\hline Team-level Transformational Leadership & .75 & .08 \\
\hline Team-level Diversity Climate & .95 & .04 \\
\hline Team-level Interaction (TL $x$ DC) & -.17 & .05 \\
\hline Team-level Organizational Justice & .10 & .48 \\
\hline Team-level Organizational Identity & -.09 & .63 \\
\hline
\end{tabular}

Note. Within model, $n=371$; between model, $n=419$. For the between model, predictor variables were grand-mean centered and control variables were group-mean centered to eliminate team effects, so control variables reflect individual differences. Gender was coded as $0=$ male, $1=$ female. 
Table 18. SCP Model: Multi-level Path Analysis Results for Taxonomy of Workplace Diversity Variables

\begin{tabular}{|c|c|c|}
\hline Variable and Predictor & Beta Weight & $p$ Value \\
\hline \multicolumn{3}{|l|}{ WITHIN MODEL } \\
\hline \multicolumn{3}{|l|}{ Diversity Climate } \\
\hline \multicolumn{3}{|l|}{ Control Variables } \\
\hline Gender & -.05 & .67 \\
\hline Education & -.06 & .08 \\
\hline Organizational Tenure & -.00 & .34 \\
\hline Proactive Personality & .13 & .05 \\
\hline Openness to Experience & .04 & .17 \\
\hline \multicolumn{3}{|l|}{ Main Variables } \\
\hline Transformational Leadership & .48 & .00 \\
\hline \multicolumn{3}{|l|}{ WITHIN MODEL } \\
\hline \multicolumn{3}{|l|}{ Organizational Justice } \\
\hline \multicolumn{3}{|l|}{ Control Variables } \\
\hline Gender & .11 & .43 \\
\hline Education & .05 & .19 \\
\hline Organizational Tenure & .00 & .72 \\
\hline Proactive Personality & .10 & .14 \\
\hline Openness to Experience & -.07 & .01 \\
\hline \multicolumn{3}{|l|}{ Main Variables } \\
\hline Transformational Leadership & -.32 & .25 \\
\hline Diversity Climate & .19 & .52 \\
\hline Transformational Leadership $x$ Diversity Climate & .10 & .08 \\
\hline \multicolumn{3}{|l|}{ WITHIN MODEL } \\
\hline \multicolumn{3}{|l|}{ Organizational Identity } \\
\hline \multicolumn{3}{|l|}{ Control Variables } \\
\hline Gender & .01 & .92 \\
\hline Education & .01 & .64 \\
\hline Organizational Tenure & .00 & .06 \\
\hline Proactive Personality & .13 & .00 \\
\hline Openness to Experience & -.05 & .01 \\
\hline \multicolumn{3}{|l|}{ Main Variables } \\
\hline Transformational Leadership & .28 & .11 \\
\hline Diversity Climate & .32 & .08 \\
\hline Transformational Leadership x Diversity Climate & -.02 & .62 \\
\hline Organizational Justice & .15 & .00 \\
\hline
\end{tabular}

Note. $n=371$. 
Table 19. SCP Model: Multi-level Path Analysis Results for Supervisor-rated Creativity

\begin{tabular}{|c|c|c|}
\hline Variable and Predictor & Beta Weight & $p$ Value \\
\hline \\
\hline \multirow{2}{*}{\multicolumn{3}{|c|}{$\begin{array}{l}\text { Supervisor-rated Creative Performance } \\
\text { Control Variables }\end{array}$}} \\
\hline & & \\
\hline Gender & -.22 & .03 \\
\hline Education & .05 & .06 \\
\hline Organizational Tenure & .00 & .05 \\
\hline Proactive Personality & .04 & .45 \\
\hline Openness to Experience & .02 & .75 \\
\hline \multicolumn{3}{|l|}{ Main Variables } \\
\hline Transformational Leadership & .16 & .50 \\
\hline Diversity Climate & .23 & .36 \\
\hline Transformational Leadership x Diversity Climate & -.05 & .32 \\
\hline Organizational Justice & -.01 & .74 \\
\hline Organizational Identity & .16 & .01 \\
\hline \multicolumn{3}{|l|}{ BETWEEN MODEL } \\
\hline \multicolumn{3}{|l|}{ Supervisor-rated Creative Performance } \\
\hline \multicolumn{3}{|l|}{ Control Variables } \\
\hline Gender & -.49 & .07 \\
\hline Education & 17 & .01 \\
\hline Organizational Tenure & .00 & .73 \\
\hline Proactive Personality & .57 & .00 \\
\hline Openness to Experience & -.09 & .24 \\
\hline \multicolumn{3}{|l|}{ Main Variables } \\
\hline Team-level Transformational Leadership & 68 & .34 \\
\hline Team-level Diversity Climate & 1.02 & .16 \\
\hline Team-level Interaction (TL x DC) & -.15 & .30 \\
\hline Team-level Organizational Justice & -.01 & .98 \\
\hline Team-level Organizational Identity & -.37 & 19 \\
\hline
\end{tabular}

Note. Within model, $n=371$; between model, $n=419$. For the between model, predictor variables were grand-mean centered and control variables were group-mean centered to eliminate team effects, so control variables reflect individual differences. Gender was coded as $0=$ male, $1=$ female. 
Table 20. Results of Indirect Effects Testing

\begin{tabular}{lcc}
\hline Indirect Effects Path & Beta & $p$ - \\
& Weight & Value \\
\hline
\end{tabular}

HYPOTHESIZED INDIRECT EFFECTS

Single-level ECP Model

H1: Indirect effect of TL on CP through DC

H3. Indirect effect of TL XDC on CP through $\mathrm{OJ} \quad-.04 \quad-.04$

$\mathrm{H} 3 \mathrm{e}$ : Indirect effect of DC on CP through OJ $\quad-.02 \quad .56$

H4: Indirect effect of TLxDC on CP through ID $\quad \begin{array}{ll}.02 & .55\end{array}$

H4f: Indirect effect of DC on CP though ID $\quad .03 \quad .34$

\section{Multi-level ECP Model}

H1: Indirect effect of TL on CP through DC

$-.14 \quad .16$

H3: Indirect effect of TLxDC on CP through OJ $\quad-.00 \quad .47$

H3e: Indirect effect of DC on CP through OJ $\quad-.01 \quad 59$

H4: Indirect effect of TLxDC on CP through ID $\quad-.00 \quad-55$

H4f: Indirect effect of DC on CP though ID $\quad .04 \quad .20$

\section{Multi-level SCP Model}

H1: Indirect effect of TL on CP through DC

H3: Indirect effect of TLxDC on CP through OJ $\quad-.00 \quad-86$

H3e: Indirect effect of DC on CP through OJ $\quad-.03 \quad-22$

H4: Indirect effect of TLxDC on CP through ID $\quad-.00 \quad 54$

H4f: Indirect effect of DC on CP though ID $\quad .05 \quad .16$

\section{EXPLORATORY INDIRECT EFFECTS}

\section{Single-level ECP}

$\begin{array}{lll}\text { Indirect effect of TL on CP through ID } & .02 & .34\end{array}$

$\begin{array}{lll}\text { Indirect effect of OJ on CP through ID } & .01 & .35\end{array}$

Multi-level ECP

Indirect effect of TL on CP through ID $\quad \begin{array}{ll}.03 & .19\end{array}$

$\begin{array}{lll}\text { Indirect effect of } \mathrm{OJ} \text { on } \mathrm{CP} \text { through ID } & .02 & .09\end{array}$

\section{Single-level SCP}

Indirect effect of TL on CP through ID $\quad \begin{array}{ll}.03 & .21\end{array}$

$\begin{array}{lll}\text { Indirect effect of OJ on CP through ID } & .02 & .22\end{array}$

Multi-level SCP

$\begin{array}{lll}\text { Indirect effect of TL on CP through ID } & .04 & .17\end{array}$

$\begin{array}{lll}\text { Indirect effect of OJ on CP through ID } & .02 & .05\end{array}$

Note. $\mathrm{ECP}=$ employee self-rate creative performance. $\mathrm{SCP}=$ supervisor-rated creative performance. $\mathrm{CP}=$ creative performance. $\mathrm{H}=$ hypothesis. $\mathrm{TL}=$ transformational leadership. $\mathrm{DC}=$ diversity climate. $\mathrm{TLxDC}=$ the interaction of transformational leadership and diversity climate. $\mathrm{OJ}=$ organizational justice. ID $=$ organizational identity. 
Table 21. Exploratory SCP Model: Multi-level Path Analysis Results for Taxonomy of Workplace Diversity Variables

\begin{tabular}{|c|c|c|}
\hline Variable and Predictor & Beta Weight & $p$ Value \\
\hline \multicolumn{3}{|l|}{ WITHIN MODEL } \\
\hline \multicolumn{3}{|l|}{ Diversity Climate } \\
\hline \multicolumn{3}{|l|}{ Control Variables } \\
\hline Gender & -.05 & .60 \\
\hline Education & -.06 & .04 \\
\hline Organizational Tenure & -.00 & .24 \\
\hline Proactive Personality & .13 & .13 \\
\hline Openness to Experience & .04 & .25 \\
\hline \multicolumn{3}{|l|}{ Main Variables } \\
\hline Transformational Leadership & .48 & .00 \\
\hline \multicolumn{3}{|l|}{ WITHIN MODEL } \\
\hline \multicolumn{3}{|l|}{ Organizational Justice } \\
\hline \multicolumn{3}{|l|}{ Control Variables } \\
\hline Gender & .11 & .42 \\
\hline Education & .05 & .11 \\
\hline Organizational Tenure & .00 & .71 \\
\hline Proactive Personality & .10 & .16 \\
\hline Openness to Experience & -.07 & .01 \\
\hline \multicolumn{3}{|l|}{ Main Variables } \\
\hline Transformational Leadership & -.32 & .21 \\
\hline Diversity Climate & .19 & .52 \\
\hline Transformational Leadership x Diversity Climate & .10 & .06 \\
\hline \multicolumn{3}{|l|}{ WITHIN MODEL } \\
\hline \multicolumn{3}{|l|}{ Organizational Identity } \\
\hline \multicolumn{3}{|l|}{ Control Variables } \\
\hline Gender & .01 & .91 \\
\hline Education & .01 & .72 \\
\hline Organizational Tenure & .00 & .08 \\
\hline Proactive Personality & .13 & .01 \\
\hline Openness to Experience & -.05 & .02 \\
\hline \multicolumn{3}{|l|}{ Main Variables } \\
\hline Transformational Leadership & .28 & .15 \\
\hline Diversity Climate & .32 & .12 \\
\hline Transformational Leadership x Diversity Climate & -.02 & .63 \\
\hline Organizational Justice & .15 & .00 \\
\hline
\end{tabular}

Note. $n=371$. 
Table 22. Exploratory SCP Model: Multi-level Path Analysis Results for Self-rated Creativity

\begin{tabular}{lcc}
\hline Variable and Predictor & Beta Weight & $p$ Value \\
\hline & & \\
WITHIN MODEL & & \\
Self-rated Creative Performance & & \\
Control Variables & -.02 & .77 \\
Gender & .05 & .01 \\
Education & .00 & .00 \\
Organizational Tenure & .36 & .00 \\
Proactive Personality & .02 & .74 \\
Openness to Experience & & \\
Main Variables & -.42 & .07 \\
Transformational Leadership & -.52 & .05 \\
Diversity Climate & .11 & $\mathbf{. 0 1}$ \\
Transformational Leadership x Diversity Climate & -.04 & .16 \\
Organizational Justice & .11 & .08 \\
Organizational Identity & & \\
\hline
\end{tabular}

Note. $n=371$. 
Table 23. Exploratory SCP Model: Multi-level Path Analysis Results for Supervisorrated Creativity

\begin{tabular}{lcc}
\hline Variable and Predictor & Beta Weight & $p$ Value \\
\hline & & \\
WITHIN MODEL & & \\
Supervisor-rated Creative Performance & & \\
Control Variables & -.21 & .00 \\
Gender & .04 & .23 \\
Education & .00 & .13 \\
Organizational Tenure & -.08 & .03 \\
Proactive Personality & -.00 & .94 \\
Openness to Experience & & \\
Main Variables & .28 & .21 \\
Transformational Leadership & .39 &. $\mathbf{1 2}$ \\
Diversity Climate & -.08 &. .07 \\
Transformational Leadership x Diversity Climate & .01 & .82 \\
Organizational Justice & $\mathbf{. 1 2}$ & $\mathbf{. 0 2}$ \\
Organizational Identity &. $\mathbf{0 7}$ & $\mathbf{. 0 0}$
\end{tabular}

\section{BETWEEN MODEL}

Supervisor-rated Creative Performance

Control Variables

Gender

Education

Organizational Tenure

$\begin{array}{ll}-.78 & .20\end{array}$

Proactive Personality

$.11 \quad .39$

Openness to Experience

$-.00$

$1.06 \quad .02$

Main Variables

$\begin{array}{lcc}\text { Team-level Transformational Leadership } & -3.27 & \mathbf{. 0 5} \\ \text { Team-level Diversity Climate } & -3.62 & \mathbf{. 0 4} \\ \text { Team-level Interaction (TL x DC) } & .72 & \mathbf{. 0 4} \\ \text { Team-level Organizational Justice } & -.74 & .20 \\ \text { Team-level Organizational Identity } & .23 & .73\end{array}$

Note. Within model, $n=371$. Between model, $n=419$. For the between model, predictor variables were grand-mean centered and control variables were group-mean centered to eliminate team effects, so control variables reflect individual differences. Gender was coded as $0=$ male, $1=$ female. 
Figure 1. Hypothesized Relationships between Leadership, Diversity, and Creativity

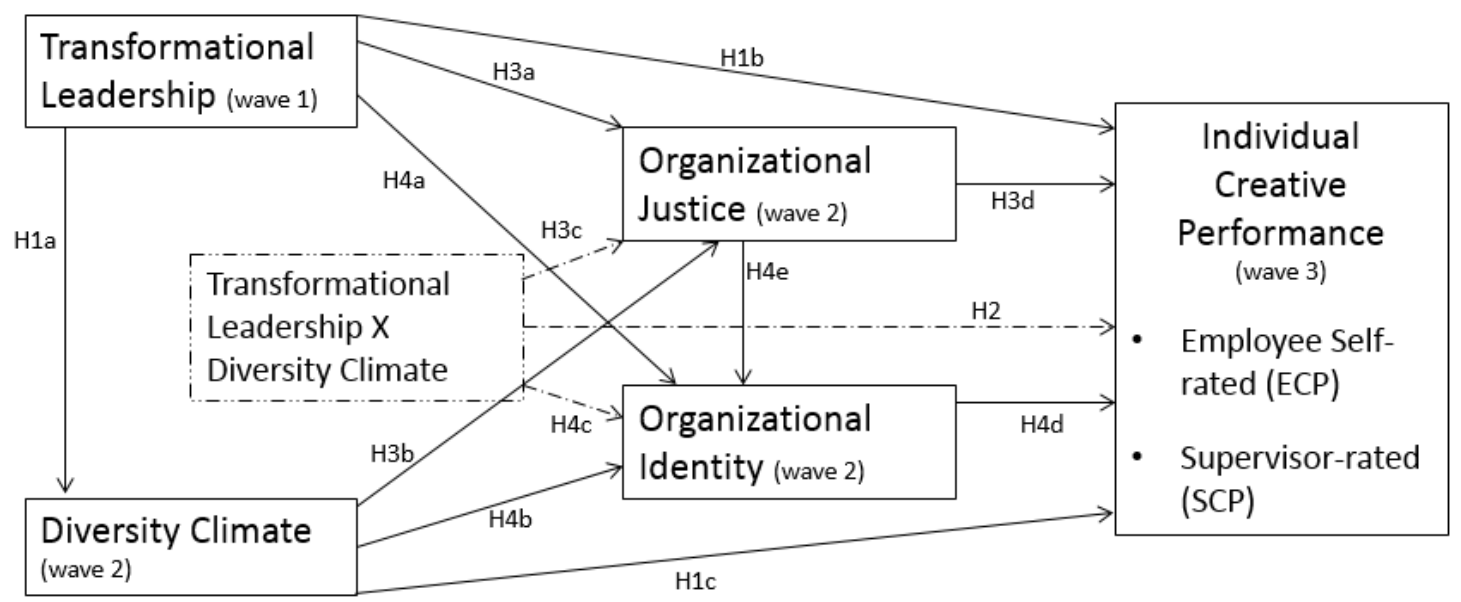

Note. All main effect hypotheses are labeled in the figure (mediation hypotheses are not labeled). The dashed and dotted lines indicated the interaction hypotheses. 
Figure 2. Expanded Componential Theory of Creativity

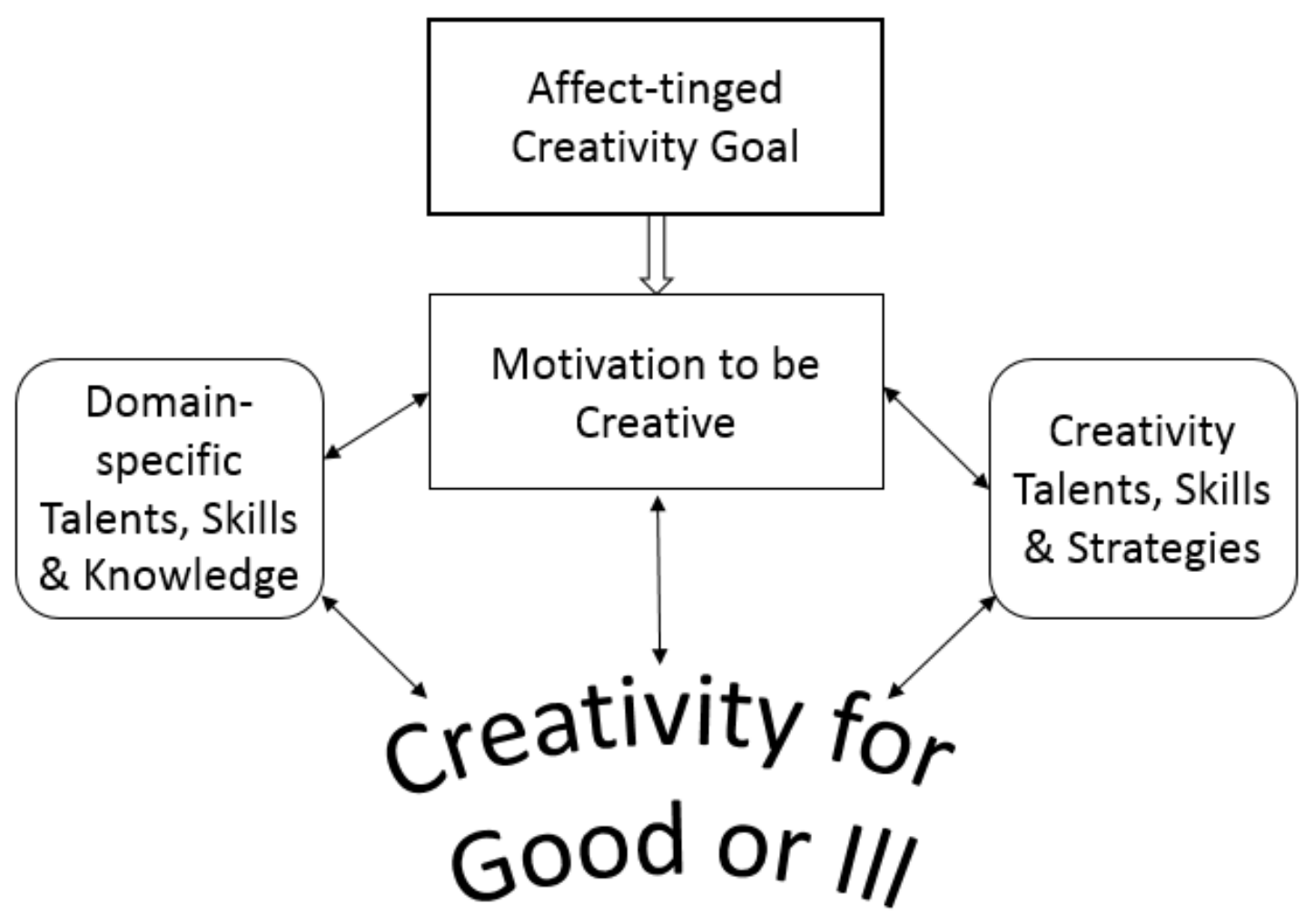


Figure 3. ECP Model: Single-level Path Analysis Results

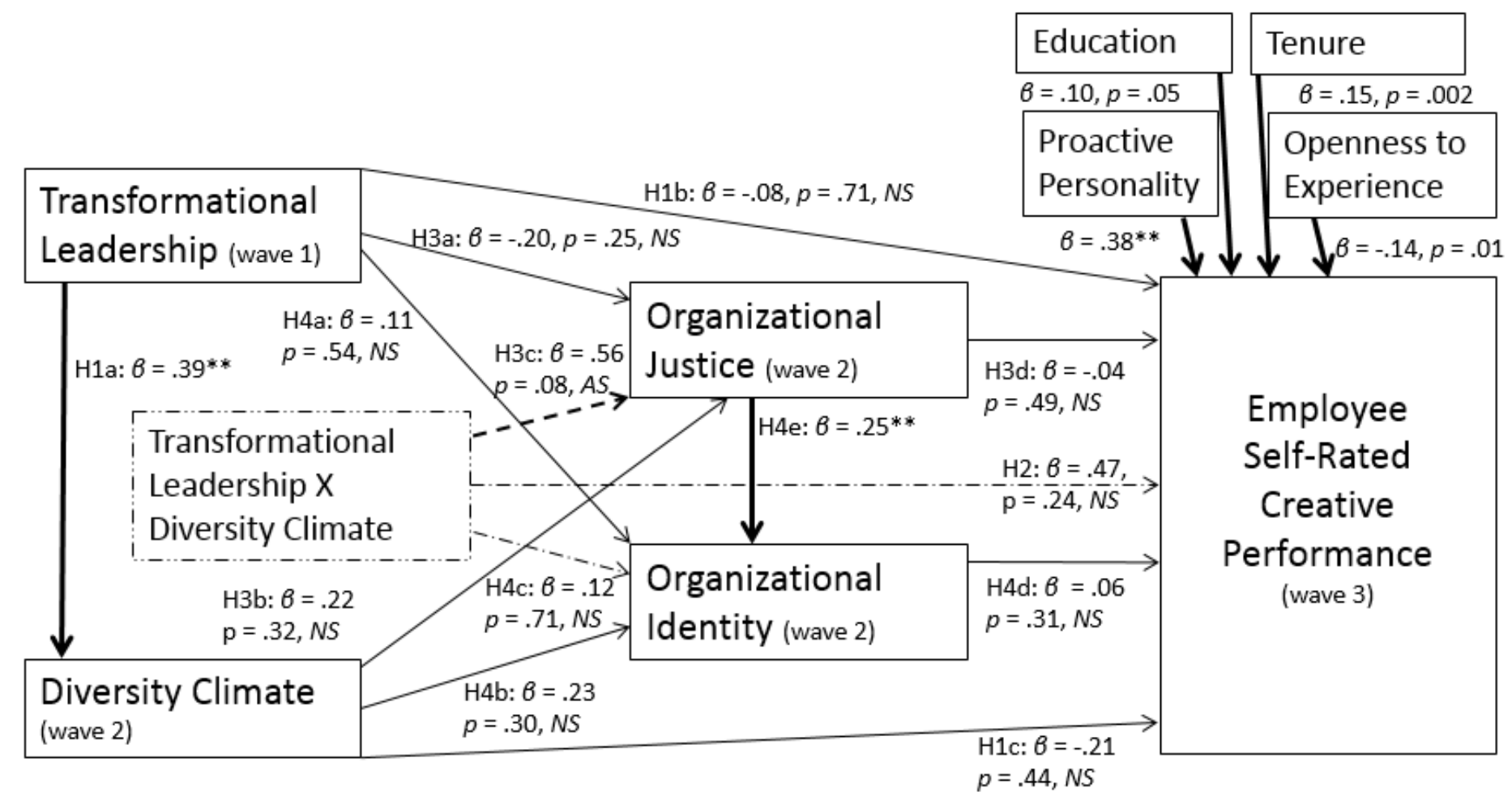

Note. ECP = employee self-rated creative performance Bolded lines indicate significant pathways. Dashed lines $\overrightarrow{\mathrm{s}}$ indicate pathways that approached significance. Dashed and dotted lines indicate the interaction pathways. ** indicates $p<.001$. 
Figure 4. ECP Model: Multi-level Path Analysis Results

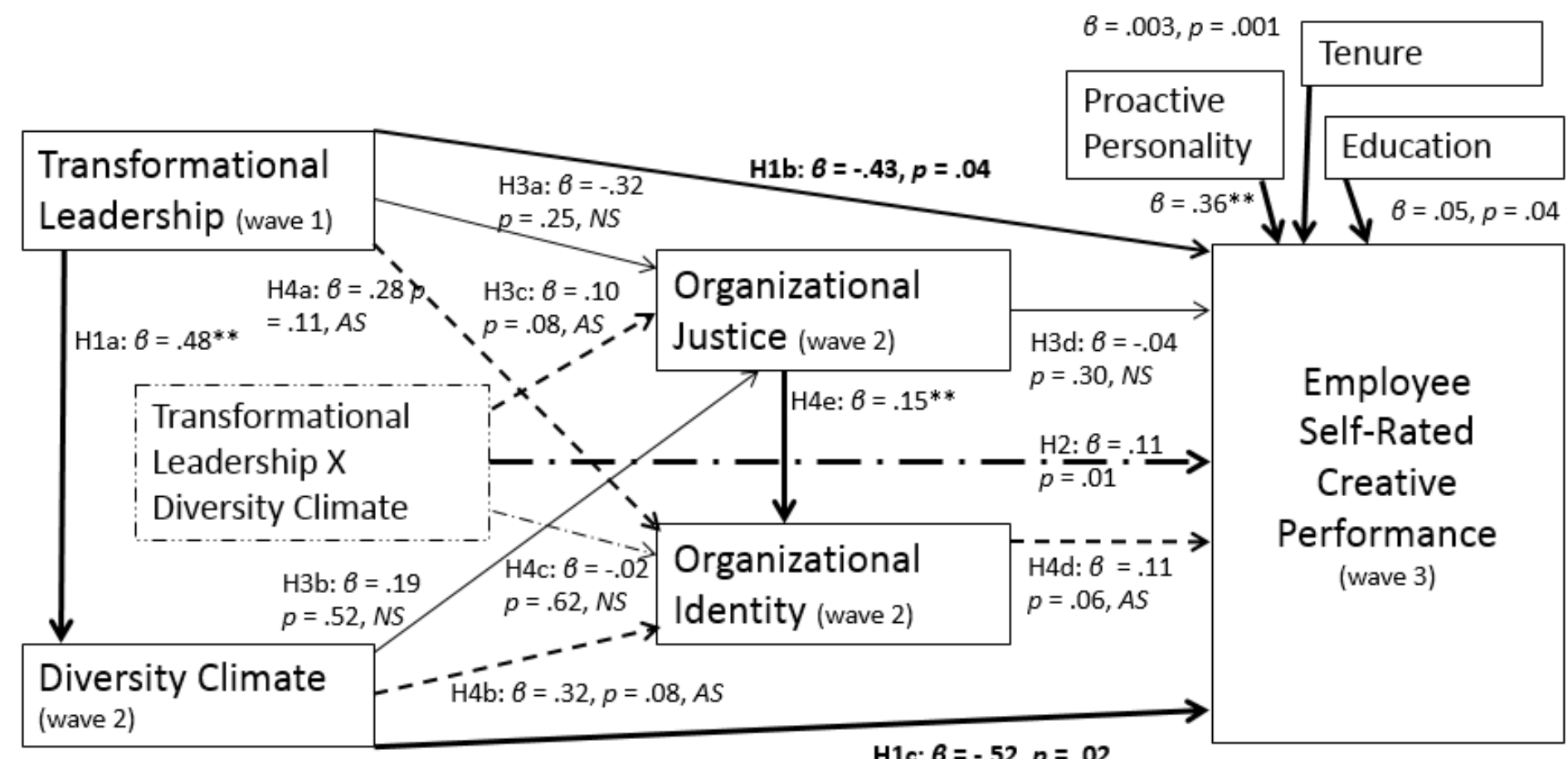

Note. ECP = employee self-rated creative performance. Bolded lines indicate significant pathways. Dashed lines indicate pathways that approached significance. Dashed and dotted lines indicate the interaction pathways. 
Figure 5. SCP Model: Multi-level Path Analysis Results

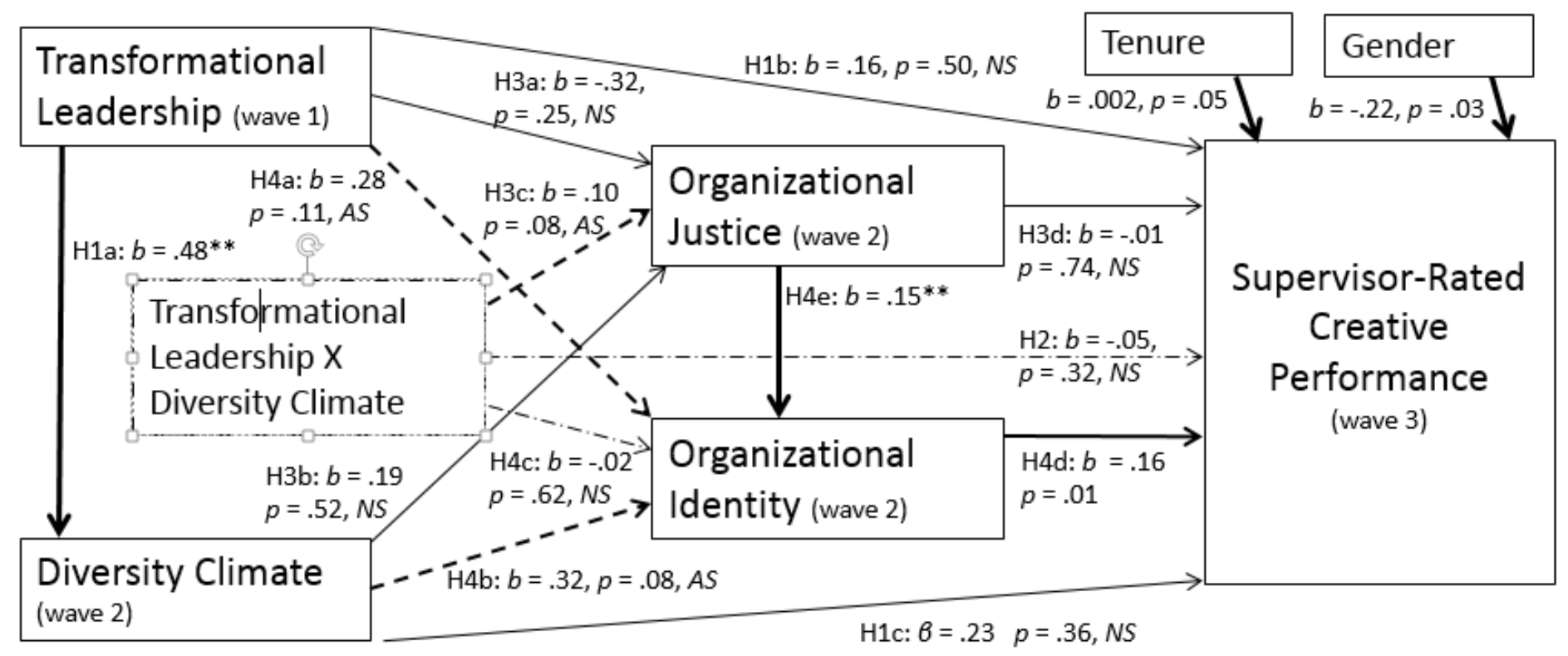

Note. SCP = supervisor-rated creative performance. Bolded lines indicate significant pathways. Dashed lines indicate pathways that approached significance. Dashed and dotted lines indicate the interaction pathways ** indicates $p<.001$. 
Figure 6. Exploratory Multilevel Path Analysis Results with ECP Predicting SCP

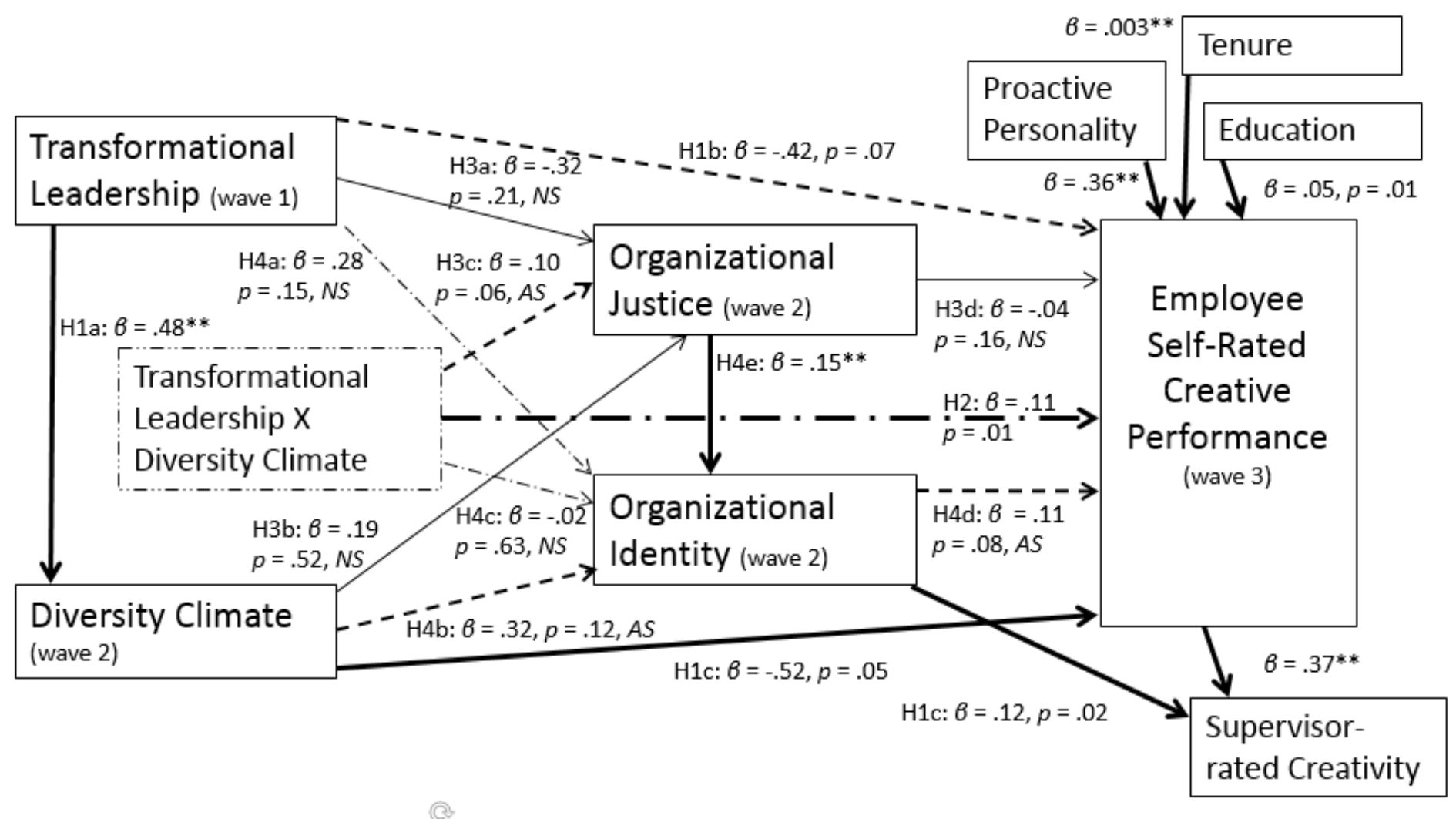

Note. $\mathrm{ECP}=$ employee self-rated creative performance. $\mathrm{SCP}=$ supervisor-rated creative performance. Bolded

lines indicate significant pathways. Dashed lines indicate pathways that approached significance. Dashed and dotted lines indicate the interaction pathways. ** indicates $p<.001$. 
Figure 7. ECP Model: Interaction of Transformational Leadership and Diversity Climate in Predicting Organizational Justice

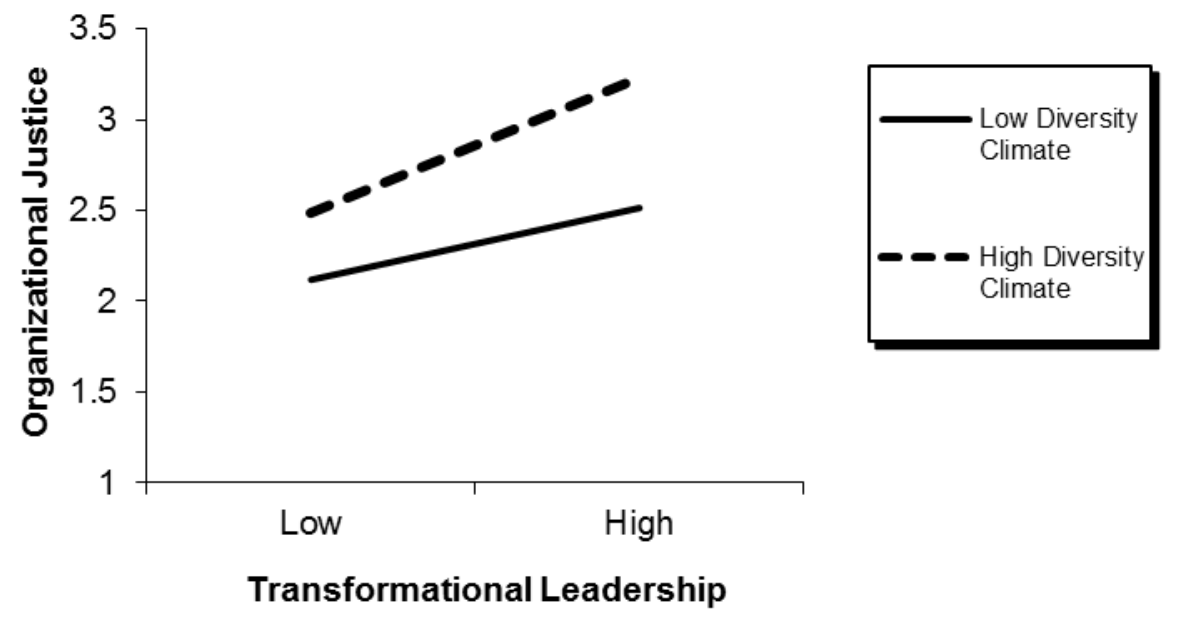

Note. ECP = employee self-rated creative performance. 
Figure 8. Multilevel ECP Model: Interaction of Transformational Leadership and Diversity Climate in Predicting ECP

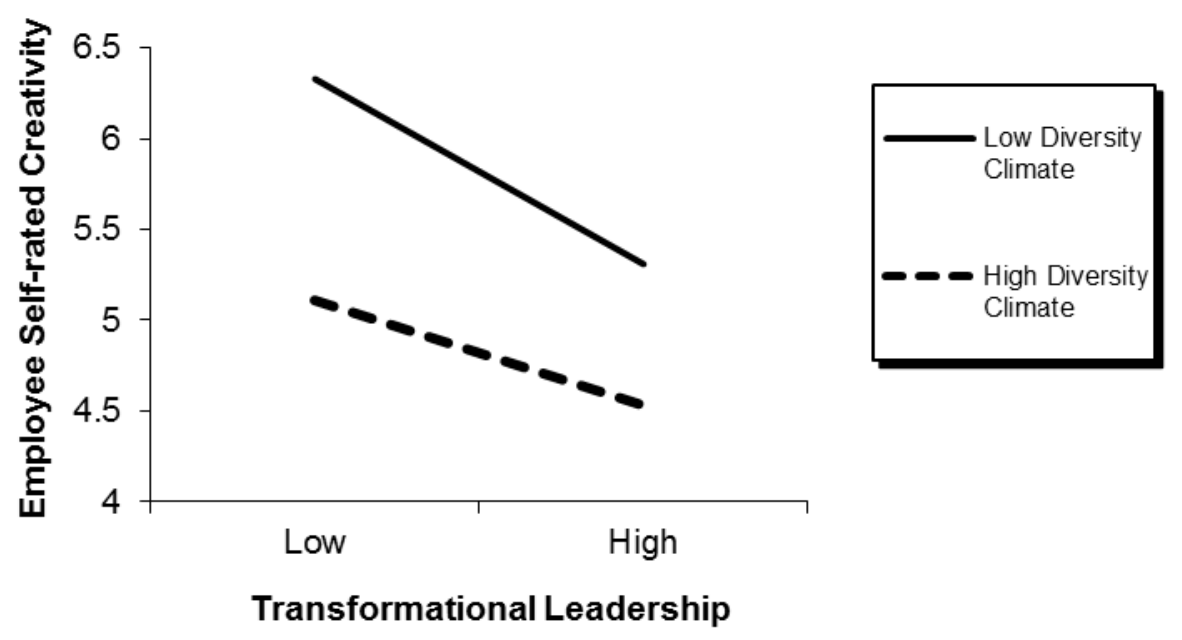

Note. $\mathrm{ECP}=$ employee self-rated creative performance. 


\section{References}

Ah Chong, L. M., \& Thomas, D. C. (1997). Leadership perceptions in cross-cultural context: Pakeha and Pacific Islanders in New Zealand. Leadership Quarterly, 8, 275-293.

Amabile, T. M. (1979). Effects of external evaluation on artistic creativity. Journal of Personality and Social Psychology, 37, 221-233.

Amabile, T. M. (1983). The social psychology of creativity. New York: SpringerVerlag.

Amabile, T. M. (1988). A model of creativity and innovation in organizations. In B. M. Staw, \& L. L. Cummings (Eds.) Research in organizational behavior, vol. 10, pp. 123-167. Greenwich, CT: JAI Press.

Amabile, T. M. (1996). Creativity in context: Update to "The Social Psychology of Creativity.” Boulder, CO: Westview Press.

Amabile, T. M., \& Conti, H. (1999). Changes in the work environment for creativity during downsizing. Academy of Management Journal, 42, 630-640.

Amabile, T. M., Conti, R., Coon, H., Lazenby, J., \& Herron, M. (1996). Assessing the work environment for creativity. Academy of Management Journal, 39, 11541184.

Amabile, T. M., Goldfarb, P., \& Brackfield, S. C. (1990). Social influences on creativity: Evaluation, coaction, and surveillance. Creativity Research Journal, 3, 6-21.

Amabile, T. M., \& Gryskiewicz, N. D. (1989). The creative environment scales: Work environment inventory. Creativity Research Journal, 2, 231-252. 
Amabile, T. M., Schatzel, E. A., Moneta, G. B., \& Kramer, S. J. (2004). Leader behaviors and the work environment for creativity: Perceived leader support. Leadership Quarterly, 15, 5-32.

Anderson, N., De Dreu, C. K. W., \& Nijstad, B. A. (2004). The routinization of innovation research: A constructively critical review of the state-of-the-science. Journal of Organizational Behavior, 25, 147-173.

Andrews, F. M., \& Farris, F. (1967). Supervisory practices and innovation in scientific teams. Personnel Psychology, 20, 497-515.

Aryee, S., Walumbwa, F. O., Zhou, Q., \& Hartnell, C. A. (2012). Transformational Leadership, Innovative Behavior, and Task Performance: Test of Mediation and Moderation Processes. Human Performance, 25:1, 1-25.

Ashforth, B. E., \& Mael, F. (1989). Social identity theory and the organization. Academy of Management Review, 14, 20-39.

Avery, D. R.,\&McKay, P. F. (2010). Doing diversity right: An empirically based approach to effective diversity management. In G. P. Hodgkinson \& J. K. Ford (Eds.), International review of industrial and organizational psychology, Vol. 25 (pp. 227-252). Chichester, UK: John Wiley \& Sons.

Avolio, B. J., \& Bass, B. M. (2004). MLQ-Multifactor leadership questionnaire. Menlo Park, CA: Mind Garden.

Baba, V. V, Tourigny, L., Wang, X., \& Liu, W. (2009). Proactive Personality and Work Performance in China: The moderating Effects of Emotional Exhaustion and 
Perceived Safety Climate. Canadian Journal of Administrative Sciences, 26: 2337. DOI: $10.1002 /$ CJAS.90.

Barling, J., Christie, A., \& Hoption, C. (2011). Leadership. In S. Zedeck (Ed.), APA handbook of industrial and organizational psychology, Vol. 1: Building and developing the organization (pp. 183-240). APA Handbooks in Psychology, Washington, DC, US: American Psychological Association.

Barcikowski, R. S. (1981). Statistical power with group mean as the unit of analysis. Journal of Educational Statistics, 6, 267-285.

Barron, R. M. \& Kenny, D. A. (1986). The Moderator-Mediator Variable Distinction in Social Psychological Research: Conceptual, Strategic, and Statistical Considerations. Journal of Personality and Social Psychology, 51 (6), 1173-1182.

Bartis, S., Szymanski, K., \& Harkins, S. G. (1988). Evaluation and performance: A two-edged knife. Personality and Social Psychology Bulletin, 14, 242-251.

Bass, B. M. (1985). Leadership and performance beyond expectations. New York, NY: Free Press.

Bass, B. M. (1997). Does the transactional-transformational leadership paradigm transcend organizational and national boundaries? American Psychologist, 52, $130-139$.

Bass, B.M., \& Avolio, B.J. (1989). The Multifactor Leadership Questionnaire. Consulting Psychologists Press, Palo Alto, CA.

Bass, B. M., \& Avolio, B.J. (1990). Manual for the Multifactor Leadership Questionnaire. Palo Alto, CA: Consulting Psychologists Press. 
Bass, B. M., Avolio, B. J., Jung, D. I., \& Berson, Y. (2003). Predicting unit performance by assessing transformational and transactional leadership. Journal of Applied Psychology, 88, 207-218.

Bass, B. M., \& Riggio, R. E. (2006). Transformational leadership (2nd ed.). Mahwah, NJ: Erlbaum.

Bateman, T. S., O’Neill, H., \& Kenworthy-U'Ren, A. (2002). A hierarchical taxonomy of top managers' goals. Journal of Applied Psychology, 87 (6), 1134-1148.

Bauer, T. N., Bodner, T., Erdogan, B., Truxillo, D. M., \& Tucker, J. S. (2007). Newcomer adjustment during organizational socialization: A meta-analytic review of antecedents, outcomes, and methods. Journal of Applied Psychology, $92,707-721$.

Beechler, S. \& Javidan, M. (2007). Leading with a global mindset. Advances in International Management, 19, 131-169.

Bell, S. T. (2007). Deep-level composition variables as predictors of team performance: A meta-analysis. Journal of Applied Psychology, 92, 595-615.

Bell, S. T., Villado, A. J., Lukasik, M. A., Belau, L., \& Briggs, A. L. (2011). Getting specific about demographic diversity variable and team performance relationships: A meta-analysis. Journal of Management, 37(3), 709-743.

Bird, A., \& Osland, J. S. (2004). Global competencies: An introduction. In H.W. Lane, M. L. Maznevski, M. E. Mendenhall, \& J. McNett (Eds.), Handbook of global management: A guide to managing complexity (pp. 57-80). London: Blackwell Publishing. 
Bliese, P. D., \& Jex, S. M. (2002). Incorporating a multilevel perspective into occupational stress research: Theoretical, methological, and practical implications. Journal of Occupational Health Psychology (7), 265-276.

Bowers, C. A., Pharmer, J. A., \& Salas, E. (2000). When member homogeneity is needed in work teams: A meta-analysis. Small Group Research, 31, 305-327.

Boone, C. A. J. J., van Olffen, W., van Witteloostuijn, A., \& de Brabander, B. (2004). The genesis of top management team diversity: Selective turnover among top management teams in Dutch newspaper publishing, 1970-94. Academy of Management Journal, 47, 633-656.

Brewer, M. (1991). The social self: On being the same and different at the same time. Personality and Social Psychology Bulletin, 17, 475-482.

Brislin, R. W. 1986. The wording and translation of research instruments. In W. J. Lonner \& J. W. Berry (Eds.), Field methods in cross-cultural research (pp. 137164). Beverly Hills, CA: Sage.

Brislin, R. (1993). Understanding culture's influence on behavior. Fort Worth: Harcourt. Burns, J. M. (1978). Leadership. New York, NY: Harper.

Carmeli, R., \& Schaubroeck, J. (2007). The influence of leaders' and other referents' normative expectations on individual involvement in creative work. Leadership Quarterly, 18, 35-48.

Chattopadhyay, P., Tluchowska, M., \& George, E. (2004). Identifying the ingroup: A closer look at the influence of demographic dissimilarity on employee social identity. Academy of Management Review, 29, 180-202. 
Cheek, J. M., \& Stahl, S. S. (1986). Shyness and verbal creativity. Journal of Research in Personality, 20, 51-61.

Chhokar, J. S., Brodbeck, F. C., \& House, R. J. (Eds.). (2007). Culture and leadership across the world: The globe book of in-depth studies of 25 societies. New York, NY: Erlbaum.

Cho, S., \& Mor Barak, M. E. (2008). Understanding diversity and inclusion in a perceived homogenous culture: A study of organizational commitment and job performance among Korean employees. Administration in Social Work, 32(4), 100-126.

Clark, K., \& James, K., (1999). Justice and Positive and Negative Creativity. Creativity Research Journal, 14 (4), 311-320.

Cohen-Charash, Y., \& Spector, P. E. (2001). The role of justice in organizations: Metaanalysis. Organizational Behavior and Human Decision Processes, 86 (2), 278321.

Cohen-Meitar, R., Carmeli, A., \& Waldman, D. A. (2009). Linking meaningfulness in the workplace to employee creativity: The intervening role of organizational identification and positive psychological experiences. Creativity Research Journal, 21 (4), 361-375.

Colquitt, J. A., Conlon, D. E., Wesson, M. J., Porter, C. O. L. H., Ng, K. Y. (2001). Justice at the millennium: A meta-analytic review of 25 years of organizational justice research. Journal of Applied Psychology, 86 (3), 425-445. 
Cox, T. Jr. (1993). Cultural diversity in organizations: Theory, research and practice. San Francisco, CA: Berrett-Koehler.

Cox, T. Jr. (2001). Creating the multicultural organization: A strategy for capturing the power of diversity. San Francisco, CA: Jossey-Bass.

Cropanzano, R., Li, A., \& James, K. (2007). Intraunit justice and interunit justice and the people who experience them. In F. Dansereau \& F. J. Yammarino (Eds.). Research in Multilevel Issues (Vol. 6, pp. 415-438). Englewood Cliffs, NJ: Erlbaum.

Cropley, D. H., Cropley, A., Kaufmann, J., \& Runco, M. (2010). Dark Side of Creativity. New York, NY: Cambridge University Press.

Csikszentmihalyi, M. (1999). Implications of a systems perspective for the study of creativity. In R. J. Sternberg (Ed.), The handbook of creativity (pp. 313-335). New York, NY: Cambridge University Press.

Dahm, M. J., Willems, E. P., Ivancevich, J. M. \& Graves, D. E., (2009). Development of an organizational diversity needs analysis instrument. Journal of Applied Social Psychology, 39, 283-318.

Dahlin, K. B., Weingart, L. R., \& Hinds, P. J. (2005). Team diversity and information use. Academy of Management Journal, 48, 1107-1123.

Dass, P., \& Parker, B. (1999). Strategies for managing human resource diversity: From resistance to learning. Academy of Management Executive, 13, 68-80. 
DeRue, D. S., Nahgang, J. D., \& Wellman, N. (2011). Trait and behavioral theories of leadership: an integration and meta-analytic test of their relative validity. Personnel Psychology, 64, 7-52.

De Dreu, C. K. W., \& Gelfand, M. J. (2008). Conflict in the workplace: Sources, functions, and dynamics across multiple levels of analysis. In C. K. W. De Dreu \& M. J. Gelfand (Eds.), The psychology of conflict and conflict management in organizations (pp. 3-54). New York, NY: Erlbaum.

de Wit, F. R. C., Greer, L. L., \& Jehn, K. A. (2012). The paradox of intragroup conflict: A meta-analysis. Journal of Applied Psychology, 97, 360-390.

Deci, E. L., \& Ryan, R. M. (1980). The empirical exploration of intrinsic motivational processes. In L. Berkowitz (Ed.), Advances in experimental social psychology (pp. 39-80). New York: Academic Press.

Deci, E. L., \& Ryan, R. M. (1985). Intrinsic motivation and self-determination in human behavior. New York: Plenum Press.

Den Hartog, D. N., House, R. J., Hanges, P. J., RuizQuintanilla, S. A., Dorfman, P. W., Abdalla, I. A., et al. (1999). Culturally-specific and cross-culturally generalizable implicit leadership theories: Are attributes of charismatic leadership theories universally endorsed? Leadership Quarterly, 10, 219-256.

Den Hartog, D. N., Van Muijen, J. J., \& Koopman, P. L.(1997). Transactional versus transformational leadership: An analysis of the MLQ. Journal of Occupational and Organizational Psychology, 70, 19-34. 
Drazin, R., Glynn, M., \& Kazanjian, R. (1999). Multilevel theorizing about creativity in organizations: A sensemaking perspective. Academy of Management Review, 24, $286-307$.

Drazin, R., \& Schoonhoven, C. B. (1996). Community, population, and organization effects on innovation: A multilevel perspective. Academy of Management Journal, 39, 1065-1083.1

Ellemers, N., De Gilder, D., \& Haslam, S. A. (2004). Motivating individuals and groups at work: A social identity perspective on leadership and group performance. Academy of Management Review, 29, 459-478.

Ely, R. J. (2004). A field of group diversity, participation in diversity education programs, and performance. Journal of Organizational Behavior, 25, 755-780.

Ely, R. J. \& Roberts, L. M. (2006). Shifting frames of diversity research: From difference to relationships. In A. P. Brief (Ed.) Diversity at work (pp. 175-201). New York, NY: Cambridge University Press.

Ely, R. J., \& Thomas, D. A. (2001). Cultural diversity at work: The effects of diversity perspectives on work group processes and outcomes. Administrative Science Quarterly, 46, 229-273.

Eden, D., \& Leviatan, U. (1975). Implicit leadership theory as a determinant of the factor structure underlying supervisory behavior scales. Journal of Applied Psychology, 60. $736-741$. 
Farmer, S. M., Tierney, P., \& Kung-McIntyre, K. (2003).Employee creativity in Taiwan: An application of role identity theory. Academy of Management Journal, 46, 618630.

Feist, G. J. (1998). A meta-analysis of personality in scientific and artistic creativity. Personality and Social Psychology Review, 2, 290-309.

Feist, G. J. (1999). Influence of personality on artistic and scientific creativity. In R. Sternberg (Ed.), Handbook of creativity (pp. 273-296). Cambridge, England: Cambridge University Press.

Felfe, J. (2006). Validation of a German version of the "Multifactor Leadership Questionnaire" (MLQ Form 5x Short) by Bass and Avolio (1995). Zeitschrift für Arbeits und Organisationspsychologie, 50, 61-78.

Findler, L., Wind, L., \& Mor Barak, M. E. (2007). The challenge of workforce management in a global society: Modeling the relationship between diversity, organizational culture, and employee well-being, job satisfaction and organizational commitment. Administration in Social Work, 31(3), 63-94.

Finke, R. A., Ward, T. B., \& Smith, S. M. (1992). Creative cognition: Theory, research, and applications. Cambridge, MA: MIT Press.

Finkelstein, S., \& Hambrick, D. C. (1996). Strategic leadership: Top executives and their effects on organizations. St. Paul, MN: West.

Fleishman, E.A., Quaintance, M. K., \& Broedling, L. A. (1987). Taxonomies of human performance: The description of human tasks. Orlando, FL: Academic Press. 
Florida, R. (2004). America's looming creativity crisis. Harvard Business Review, 10, 122-124.

Ford, C. M. (1996). A theory of individual creative action in multiple social domains. Academy of Management Review, 21, 1112-1142.

Frink, D. D., Robinson, R. K., Reithel, B., Arthur, M. M., Ammeter, A. P., \& Ferris, G. R. (2003). Gender demography and organization performance: A two-study investigation with convergence. Group and Organization Management, 28, 127147.

Frese, M., Teng, E., \& Wijnen, C. J. (1999). Helping to improve suggestion systems: Predictors of making suggestions in companies. Journal of Organizational Behavior, 20, 1139-1155.

Fukushige, A. \& Spicer, D. P. (2007). Leadership preferences in Japan: An exploratory study. Leadership and Organization Development Journal, 28, 508-530.

Gardner, H. (1988). Creativity: An Interdisciplinary perspective. Creativity Research Journal, 1, 8-26.

Gardner, H. 1989. To open minds. New York: Basic Books.

George, J. M., \& Zhou, J. (2001). When openness to experience and conscientiousness are related to creative behavior: An interactional approach. Journal of Applied Psychology, 86, 513-524.

Gerstner, C. R., \& Day, D. V. (1994). Cross-cultural comparison of leadership prototypes. Leadership Quarterly, 5, 121-134. 
Gibson, C. B., \& Vermeulen, F. (2003). A healthy divide: Subgroups as a stimulus for team learning behavior. Administrative Science Quarterly, 48, 202-239.

Gilbert, J. A., \& Ivancevich, J. M. (2000). Valuing diversity: A tale of two organizations. Academy of Management Executive, 14, 93-105.

Gilson, L. L. (2008). Why be creative: A review of the practical outcomes associated with creativity at the individual, group, and organizational levels. In J. Zhou \& C. E. Shalley (Eds.), Handbook of organizational creativity (pp. 303-322). New York: Erlbaum.

Gilson, L. L., \& Shalley, C. E. (2004). A little creativity goes a long way: An examination of teams' engagement in creative processes. Journal of Management, 30, 453-470.

Gough, H. G. (1979). A creativity scale for the Adjective Check List. Journal of Personality and Social Psychology, 37, 1398-1405.

Greenberg, J. (1987). A taxonomy of organizational justice theories. Academy of Management Review, 12, 9-22.

Guillaume, Y. R. F., Brodbeck, F. C., \& Riketta, M. (2012). Surface- and deep-level dissimilarity effects on social integration and individual effectiveness related outcomes in work groups: A meta-analytic integration. Journal of Occupational and Organizational Psychology, 85, 80-115.

Guillaume, Y. R. F., Dawson, J. F., Woods, S. A., Sacramento, C. A., \& West, M. A. (2013). Getting diversity at work to work: What we know and what we still don't 
know. Journal of occupational and organizational psychology (0963-1798), 86 (2), p. 123.

Guzzo, R., \& Dickson, M. (1996). Teams in organizations: Recent research on performance and effectiveness. Annual Review of Psychology, 47, 307-338.

Haq, R. (2004). International perspectives on workplace diversity. In M. S. Stockdale and F. J. Crosby (Eds.), The psychology and management of workplace diversity (pp. 277-298). Malden, MA: Blackwell.

Harrison, D. A., \& Klein, K. J. (2007). What's the difference? Diversity constructs as separation, variety, or disparity in organizations. Academy of Management Review 32(4), 1199-1228.

Hays-Thomas, R. (2004). Why now? The contemporary focus on managing diversity. In M. S. Stockdale and F. J. Crosby (Eds.), The psychology and management of workplace diversity (pp. 3-30). Malden, MA: Blackwell.

Heinitz, K., Liepmann, D., \& Felfe, J. (2005). Examining the factor structure of the MLQ: Recommendation for a reduced set of factors. European Journal of Psychological Assessment, 21, 182-190.

Hirschfeld, R. R., Jordan, M. H., Feild, H. S., Giles, W. F., \& Armenakis, A. A. (2005). Teams' female representation and perceived potency as inputs to team outcomes in a predominantly male field setting. Personnel Psychology, 58, 893-924.

Hirst, G., van Knippenberg, D., \& Zhou, J. (2009). A multi-level perspective on employee creativity: Goal orientation, team learning behavior, and individual creativity. Academy of Management Journal, 52, 2. 
Hofstede, (2001). Culture's Consequences: International Differences in Work-Related Values.

Hoffman, E. (1985). The effect of race-ratio composition on the frequency of organizational communication. Social Psychology Quarterly, 48, 17-26.

Hogan, R., \& Kaiser, R. B. (2005). What we know about leadership. Review of General Psychology, 9, 169-180.

Hogg, M. A. \& Terry, D. J. (2000). Social identity and self-categorization processes in organizational contexts. Academy of Management Review, 25, 121-140.

Holt, K., \& Seki, K. (2012). Global leadership: A developmental shift for everyone. Industrial and Organizational Psychology: Perspectives on Science and Practice, 5, 196-215.

Homan, A. C., van Knippenberg, D., Van Kleef, G. A., \& De Dreu, C. K. W. (2007). Bridging faultlines by valuing diversity: Diversity beliefs, information elaboration, and performance in diverse work groups. Journal of Applied Psychology, 92, 1189-1199.

Horwitz, S. K., \& Horwitz, I. B. (2007). The effects of team diversity on team outcomes: A meta-analytic review of team demography. Journal of Management, 33, 9671015.

House, R. J., Hanges, P. J., Javidan, M., Dorfman, P. W., \& Gupta, V. (Eds.) (2004). Culture, leadership and organizations: The GLOBE study of 62 societies. Thousand Oaks, CA: Sage. 
Hox, J. (2002). Multilevel analysis: Techniques and applications. Mahwah, NJ: Lawrence Erlbaum Associates, Inc.

Hu, L.T., \& Bentler, P. (1999). Cutoff criteria for fit indexes in covariance structure analysis: Conventional criteria versus new alternatives. Structural Equation Modeling, 6, 1-55.

Hülsheger, U. R., Anderson, N. \& Salgado, J. F. (2009). Team-level predictors of innovation at work: A comprehensive meta-analysis spanning three decades of research. Journal of Applied Psychology, 94, 1128-1145.

Ibarra, H. (1992). Homophily and differential returns: Sex differences in network structure and access in an advertising firm. Administrative Science Quarterly, 37, $422-447$.

Jackson, S. E. (1992). Consequences of group composition for the interpersonal dynamics of strategic issue processing. In P. Shrivastava, A. Huff, \& J. Dutton (Eds.), Advances in strategic management (pp. 345-382). Greenwich, CT: JAI Press.

Jackson, S. E., Brett, J. F., Sessa, V. I., Cooper, D. M., Julin, J. A., \& Peyronnin, K. (1991). Some differences make a difference: Individual dissimilarity and group heterogeneity as correlates of recruitment, promotions, and turnover. Journal of Applied Psychology, 76, 675-689.

Jackson, S. E., \& Chung, Y. (2008). The people make the place complicated. In D. B. Smith (Ed.), The people make the place: Dynamic linkages between individuals and organizations (pp. 37-52). Mahwah, NJ: Erlbaum. 
Jackson, S. E., Hitt, M. A., \& DeNisi, A. S. (Eds.) (2003). Managing knowledge for sustained competitive advantage: Designing strategies for effective human resource management. San Francisco: Jossey-Bass.

Jackson, S. E., Joshi, A., \& Erhardt, N. L. (2003). Recent research on team and organizational diversity: SWOT analysis and implications. Journal of Management, 29, 801-830.

Jackson, S. E., \& Joshi, A. (2004). Diversity in social context: A multiattribute, multilevel analysis of team diversity and sales performance. Journal of Organizational Behavior, 25, 675-702.

Jackson, S. E., \& Joshi, A. (2011). Work Team Diversity. In S. Zedeck (Ed.), APA handbook of industrial and organizational psychology, Vol. 1: Building and developing the organization (pp. 651-686). APA Handbooks in Psychology, Washington, DC, US: American Psychological Association.

Jackson, S. E., Joshi, A., \& Erhardt, N. L. (2003). Recent research on team and organizational diversity: SWOT analysis and implications. Journal of Management, 29, 801-830.

Jackson, S. E., May, K. E., \& Whitney, K. (1995). Understanding the dynamics of diversity in decision-making teams. In R. A. Guzzo, E. Salas, \& Associates (Eds.), Team effectiveness and decision making in organizations (pp. 204-261). San Francisco, CA: Jossey-Bass Publishers.

Janssen O (2000) Job demands, perceptions of effort-reward fairness and innovative work behavior. Journal of Occupational and Organizational Psychology 73: 287-302. 
James, K. (in press). Organizational (In)Justice and Creativity. Creativity Research Journal.

James, K. (in press). Culture and organizational justice: State of the literature and suggestions for the future directions. Handbook of Organizational Justice.

James, K. \& Clark, K. (2009). Organizational Justice Effects on Positive and Negative Creativity. Manuscript under review, Portland State University.

James, K., Clark, K., \& Cropanzano, R. (1999). Positive and negative creativity in groups, institutions, and organizations: A model and theoretical extension. Creativity Research Journal, 12, 211-227.

James, K., \& Drown, D. (2008). Whether "malevolent" or "negative" creativity is relevant to terrorism prevention: Lessons from 9/11 and hazardous material trucking. Creativity Research Journal, 20(2), 120-127.

James, K. \& Lahti, K. (2011a). Organizational vision and system influences on employee inspiration and organizational performance. Creativity and Innovation Management Journal. 20, 1-14.

James, K. \& Lahti, K. (2011b). Multi-level inspirational leadership, organizational vision supports, and employee vision inspiration. Manuscript under review, Portland State University.

James, K., Lovato, C., \& Cropanzano, R. (1994). Correlational and known-group comparison validation of a workplace prejudice/discrimination inventory. Journal of Applied Social Psychology, 24, 1573-1592. 
James, K., \& Taylor, A. S. (2010). Positive Creativity and Negative Creativity (and Unintended Consequences). In D. Copley, A. Cropley, J. Kaufmann, and M. Runco (Eds.), Dark Side of Creativity (pp. 33-56). New York, NY: Cambridge University Press.

James, K., Yao, X., \& Lahti, K. (in press). Multi-level inspirational leadership, organizational vision supports, and employee vision inspiration and creativity.

James, L. R. (1982). Aggregation bias in estimates of perceptual agreement. Journal of Applied Psychology, 67, 219-229.

James, L. R., \& Brett, J. M. (1984). Mediators, moderators, and tests for mediation. Journal of Applied Psychology, 69, 307-321.

Javidan, M., Dorfman, P. W., de Luque, M. S., House, R. J., 2006). In the eye of the beholder: Cross cultural lessons in leadership from Project GLOBE. Academy of Management: Perspectives, February, 60-90.

Jehn, K. A. (1995). A multimethod examination of the benefits and detriments of intragroup conflict. Administrative Science Quarterly, 40, 256-282.

Jehn, K. A., \& Bendersky, C. (2003). Intragroup conflict in organizations: A contingency perspective. Research in Organizational Behavior, 25, 189-244.

Jehn, K. A., \& Mannix, E. A. (2001). The dynamic structure of conflict: A longitudinal study of intragroup conflict and group performance. Academy of Management Journal, 44, 238-251.

Johnson, R. E. \& Lord, R. G. (2010). Implicit effects of justice on self-identity. Journal of Applied Psychology, 95 (4), 681-695. 
Joshi, A., Liao, H., \& Jackson, S. E. (2006). Cross-level effects of workplace diversity on sales performance and pay. Academy of Management Journal, 49, 459-481.

Joshi, A., \& Roh, H. (2007). Context matters: A multilevel framework for work team diversity research. In J. J. Martocchio (Ed.), Research in personnel and human resources management (Vol. 26, pp. 1-48). Greenwich, CT: JAI Press.

Joshi, A., \& Roh, H. (2009). The role of context in work team diversity research: A metaanalytic review. Academy of Management Journal, 52, 599-627.

Judge, T. A., \& Bono, J. E. (2000). Five-factor model of personality and transformational leadership. Journal of Applied Psychology, 85, 751-765.

Judge, T. A., \& Piccolo, R. F. (2004). Transformational and transactional leadership: A meta-analytic test of their relative validity. Journal of Applied Psychology, 89, $755-768$.

Judy, R. W., \& D’Amico, C. (1997). Workforce 2020: Work and workers in the 21st century. Indianapolis, IN: Hudson Institute.

Kark, R., \& Carmeli, A. (2008). Alive and creating: The mediating role of vitality and aliveness in the relationship between psychological safety and creative work involvement. Journal of Organizational Behavior, 30, 785-804.

Kark, R., Shamir, B., \& Chen, G. (2003). The two faces of transformational leadership: Empowerment and dependency. Journal of Applied Psychology, 88, 246-255.

Kearney, E., \& Gebert, D. (2009). Managing diversity and enhancing team outcomes: The promise of transformational leadership. Journal of Applied Psychology, 94, $77-89$. 
Kochan, T., Bezrukova, K., Ely, R., Jackson, S., Joshi, A., Jehn, K., et al. (2003). The effects of diversity on business performance: Report of the Diversity Research Network. Human Resource Management, 42, 3-21.

Koper, G., van Knippenberg, D., Bouhuijs, F., Vermunt, R., \& Wilke, H. (1993). Procedural fairness and self esteem. European Journal of Social Psychology, 23, 313-325.

Kossek, E. E., Markel, K. S., \& McHugh, P. P. (2003). Increasing diversity as an HRM change strategy. Journal of Organizational Change Management, 16, 328-352.

Kossek, E. E., \& Zonia, S. C. (1993). Assessing diversity climate: A field study of reactions to employer efforts to promote diversity. Journal of Organizational Behavior, 14, 61-81.

Kristof, A. L. (1996). Person-organization fit: An integrative review of its conceptualization, measurement, and implication. Personnel Psychology, 49(1), $1-49$.

Kristof-Brown, A. L., Zimmerman, R. D., \& Johnson, E. C. 2005. Consequences of individuals' fit at work: A meta-analysis of person-job, person-organization, person-group, and person-supervisor fit. Personnel Psychology, 58, 281-342.

Kulik, C. T., \& Roberson, L. (2008). Diversity initiative effectiveness: What organizations can (and cannot) expect from diversity recruitment, diversity training, and formal mentoring programs. In A. P. Brief (Ed.), Diversity at work (pp. 265-317). Cambridge, England: Cambridge University Press. 
Lane, H.W., M.L. Maznevski, M.E. Mendenhall \& J. McNett. (eds). 2004. The Blackwell handbook of global management: A guide to managing complexity. London Blackwell.

Lau, D. C., \& Murnighan, J. K. (1998). Demographic diversity and faultlines: The compositional dynamics of organizational groups. Academy of Management Review, 23, 325-340.

Leong, L. Y. C., \& Fischer, R. (2011). Is transformational leadership universal? A metaanalytic investigation of multifactor leadership questionnaire means across cultures. Journal of Leadership \& Organizational Studies, 18 (2), 164-174.

Lester, S. (2006). Diversity and profitability: Making the connection (Executive Action Report No. A-0175-06-EA). New York: The Conference Board, Inc.

Levy, O., Beechler, S., Taylor, S., \& Boyacigiller, N. (2007). What we talk about when we talk about global mindset: Managerial cognition in Multinational Corporations. Journal of International Business Studies, 38(2), 231-258.

Li, A. (2012). Toward a geocentric meta-paradigm of creative cognition. Paper presented at the Academy of Management Annual Meeting, Boston, MA.

Li, A., \& Cropanzano, R. (2009). Do East Asians Respond More/Less Strongly to Organizational Justice Than North Americans? A Meta-Analysis. Journal of Management Studies, 46 (5), 787-805.

Lim, B.-C., \& Ployhart, R. E. (2004). Transformational leadership: Relations to the fivefactor model and team performance in typical and maximum contexts. Journal of Applied Psychology, 89, 610-621. 
Lipponen, J., Wisse, B., \& Perala, J. (2011). Perceived justice and group identification: The moderating role of previous identification. Journal of Personnel Psychology, $10,13-23$.

Locke, E. A., \& Latham, G. P. (2002). Building a practically useful theory of goal setting and task motivation: A 35-year odyssey. American Psychologist, 57, 705-717.

Madjar, N., Oldham, G. R., \& Pratt, M. G. (2002). There's no place like home?: The contributions of work and nonwork creativity support to employees' creative performance. Academy of Management Journal, 45, 757-767.

Mainemelis C (2010) Stealing fire: Creative deviance in the evolution of new ideas. Academy of Management Review 35: 558-578.

Mangaliso, M. P., \& Nkomo, S. N. (2001). Herdbuoys-McCann Erickson's Chairman Peter Vundla on Black entrepreneurs and economic empowerment. Academy of Management Executive, 15, 16-22.

McCall, M., \& Hollenbeck, G. (2002). Developing global executives: The lessons of international experience. Boston, MA: Harvard Business School Press.

McCall, G., \& Simmons, J. L. 1978. Identities and interaction. New York: Free Press. McKay, P. F., \& Avery, D. R. (2006). What has race got to do with it? Unraveling the role of racioethnicity in job seekers' reactions to site visits. Personnel Psychology, 59, 395-429.

McKay, P. F., Avery, D. R., \& Morris, M. A. (2008). Mean racial-ethnic differences in employee sales performance: The moderating role of diversity climate. Personnel Psychology, 61, 349-374. 
McKay, P. F., Avery, D. R., Tonidandel, S., Morris, M. A., Hernandez, M., \& Hebl, M. R. (2007). Racial differences in employee retention: Are diversity climate perceptions the key? Personnel Psychology, 60, 35-62.

McGrath, J. E. (1984). Groups, interaction, and performance. Englewood Cliffs, NJ: Prentice-Hall.

McLaren, R.B. (1993). The dark side of creativity. Creativity Research Journal, 6, 137144.

Mento, A. J., Steel, R. P., \& Karren, R. J. (1987). A meta-analytic study of the effects of goal setting on task performance: 1966-1984. Organizational Behavior and Human Decision Processes, 39, 52-83.

Messick, D. M. \& Mackie, D. M. (1989). Intergroup relations. Annual Review of Psychology, 40, 51-81.

Milliken, F., \& Martins, L. (1996). Searching for common threads: Understanding the multiple effects of diversity in organizational groups. Academy of Management Review, 21, 402-433.

Mohammed, S., \& Angell, L. C. (2004). Surface- and deep-level diversity in workgroups: Examining the moderating effects of team orientation and team process on relationship conflict. Journal of Organizational Behavior, 25, 1015-1039.

Mor Barak, M. E. (2011). Managing diversity: Toward a globally inclusive workplace (Second ed.) Thousand Oaks, CA: Sage Publishing. 
Mor Barak, M. E., Cherin, D. A., \& Berkman, S. (1998). Organizational and personal dimensions of diversity climate: Ethnic and gender differences in employee perceptions. Journal of Applied Behavioral Sciences, 31, 82-104.

Mor Barak, M. E., Findler, L., \& Wind, L. (2003). Cross-cultural aspects of diversity and well-being in the workplace: An international perspective. Journal of Social Work Research and Evaluation, 4(2), 49-73.

Mor Barak, M. E., \& Levin, A. (2002). Outside the corporate mainstream and excluded from the work community: A study of diversity, job satisfaction and well-being. Community, Work \& Family, 5(2), 133-157.

Mueller, C., Finley, A., Iverson, R., \& Price, J. (1999). The effects of group racial composition on job satisfaction, organizational commitment, and career commitment: The case of teachers. Work and Occupation, 26, 187-219.

Mumford, M. D. (2011). Handbook of Organizational Creativity. London: Academic Press.

Muthén, L. K., \& Muthén, B. O. (2010). Mplus: Statistical analysis with latent variables: User's guide. Los Angeles, CA: Muthén \& Muthén.

Ng, T. W., \& Feldman, D. C. (2012). A comparison of self-ratings and non-self-report measures of employee creativity. Human Relations, 65(8), 1021-1047.

Nemeth, C. J. (1986). Differential contributions of majority and minority influence. Psychological Review, 91, 23-32.

Nemeth, C. J. (1997). Managing innovation: When less is more. California Management Review, 40, 59-74. 
Nishii, L. H., \& Raver, J. L. (2003, April). Collective climates for diversity: Evidence from a field study. Paper presented at the 18th Annual Conference of the Society for Industrial and Organizational Psychology, Orlando, FL.

Nishii, L. H., \& Özbilgin, M. F. (2007). Global diversity management: Towards a conceptual framework. International Journal of Human Resource Management, $11,1883-1894$.

Nunnally, J. C., \& Bernstein, I. H. (1994). Psychometric theory (Third ed.). New York: McGraw-Hill.

O'Connell, D., Hickerson, K, \& Pillutla, A. (2011). Organizational visioning: An integrative review. Group \& Organization Management, 36, 103-125.

O’Reilly, C. A., III, Caldwell, D. F., \& Barnett, W. P. (1989). Work group demography, social integration, and turnover. Administrative Science Quarterly, 34, 21-37.

Oh, H., Chung, M., \& Labianca, G. (2004). Group social capital and group effectiveness: The role of informal socializing ties. Academy of Management Journal, 47, 860-875.

Ohio State, Orientations to East Asia. Accessed on 4-28-13: http://people.cohums.ohiostate.edu/bender4/eall131/EAHReadings/module0 $\underline{\text { 1/module01ethnicdiversity.html. }}$.

Okuda, S. M., Runco, M. A., \& Berger, D. W. (1991). Creativity and the finding and solving of real-world problems. Journal of Psychoeducational Assessment, 9, 45-53. 
Oldham, G. R., \& Cummings, A. (1996). Employee creativity: Personal and contextual factors at work. Academy of Management Journal, 39 (3), 607634.

Osche, R. (1990). Before the gates of excellence: Factors that aid and hinder creativity. Cambridge: Cambridge University Press.

Osland, J. S., Bird, A., Mendenhall, M., \& Osland, A. (2006). Developing global leadership capabilities and global mindset: A review. In: G. Stahl \& I. Bjorkman (Eds), Handbook of research in international human resource management, (pp. 197-222). Northhampton, MA: Edward Elgar Publishing.

Osland, J. S., Taylor, S., \& Mendenhall, M. E. (2009). Global leadership: Progress and challenges. In R. S. Bhagat \& R. M. Steers (Eds). Cambridge Handbook of Culture, Organizations, and Work (pp. 245-271).

Pearsall, M. J., Ellis, A. P. J. \& Evans, J. M. (2008). Unlocking the effects of gender faultlines on team creativity: Is activation the key? Journal of Applied Psychology, 93, 225-234.

Pelled, L. H., Eisenhardt, K. M., \& Xin, K. R. (1999). Exploring the black box: An analysis of work group diversity, conflict and performance. Administrative Science Quarterly, 44, 1-28.

Pearsall, M. J., Ellis, A. P. J., \& Evans, J. M. (2008). Unlocking the effects of gender faultlines on team creativity: Is activation the key? Journal of Applied Psychology, 93, 225-234. 
Petkus, E. 1996. The creative identity: Creative behavior from the symbolic interactionist perspective. Journal of Creative Behavior, 30, 188-196.

Pfeffer, J. (1983). Organizational demography. In L. L. Cummings \& B. M. Staw (Eds.), Research in organizational behavior (Vol. 5, pp. 299-357). Greenwich, CT: JAI Press.

Pfeffer, J. (1992). Managing with power: Politics and influence in organizations. Boston, MA: Harvard Business Press.

Piliavin, J. A., \& Callero, P. L. 1991. Giving blood: The development of an altruistic identity. Baltimore: Johns Hopkins.

Pillai, R., Schriesheim, C. A., \& Williams, E. S. (1999). Fairness perceptions and trust as mediators for transformational and transactional leadership: A twosample study. Journal of Management, 25, 897-933.

Plaut, V. C., Thomas, K. M., \& Goren, M. J. (2009). Is multiculturalism or color blindness better for minorities? Psychological Science, 20, 444-445.

Podsakoff, P. M., MacKenzie, S. B., Moorman, R. H. \& Fetter, R. (1990). Transformational leader behaviors and their effects on followers' trust in leader, satisfaction and organizational citizenship behaviors. Leadership Quarterly, 1, 107-142.

Podsakoff, P. M., MacKenzie, S. B., \& Bommer, W. H. (1996). Transformational leader behaviors and substitutes for leadership as determinants of employee satisfaction, commitment, trust, and organizational citizenship behaviors. Journal of Management, 22, 259-298. 
Podsakoff, P. M., MacKenzie, S. B., Lee, J., \& Podsakoff, N. P. (2003). Common method biases in behavioral research: A critical review of the literature and recommended remedies. Journal of Applied Psychology, 88, 879.

Preacher, K. J., Rucker, D. D., \& Hayes, A. F., (2007). Addressing Moderated Mediation Hypotheses: Theory, Methods, and Prescriptions. Multivariate Behavioral Research, 42(1), 185-227.

Pugh, S. D., Dietz, J., Brief, A. P., \& Wiley, J. W. (2008). Looking inside and out: The impact of employee and community demographic composition on organizational diversity climate. Journal of Applied Psychology, 93, 6, 1422-1428.

Randel, A. E. (2002). Identity salience: A moderator of the relationship between group gender composition and work group conflict. Journal of Organizational Behavior, 23, 749-766.

Reagans, R., \& Zuckerman, E. W. (2001). Networks, diversity, and productivity: The social capital of corporate R\&D teams. Organization Science, 12, 502-517.

Reagans, R., Zuckerman, E. W., \& McEvily, B. (2004). How to make the team: Social networks vs. demography as criteria for designing effective teams. Administrative Science Quarterly, 49, 101-133.

Reiter-Palmon, R., \& Illies, J. J. (2004). Leadership and creativity: Understanding leadership from a creative problem-solving perspective. Leadership Quarterly, $15,55-77$.

Reynolds, K. J., Turner, J. C., \& Haslam, S. A. (2003). Social identity and selfcategorization theories' contribution to understanding identification, salience and 
diversity in teams and organizations. In J. T. Polzer (Ed.), Research on managing groups and teams (Vol. 5, pp. 279-304). Oxford, England: Elsevier Science.

Rich, G. (1997). The sales manager as a role model: Effects of trust, job satisfaction, and performance of salespeople. Journal of Academy of Marketing Science, $25,319-328$.

Richard, O. C., Barnett, T., Dwyer, S., \& Chadwick, K. (2004). Cultural diversity in management, firm performance, and the moderating role of entrepreneurial orientation. Academy of Management Journal, 47, 255-266.

Riley, A., \& Burke, P. J. 1995. Identities and self-verification in the small group. Social Psychology Quarterly, 58, 61-73.

Roberson, Q. R., \& Colquitt, J. A. (2005). Shared and configural justice: A social network model of justice in teams. Academy of Management Review, 30, 3 , $595-607$.

Roberson, Q. M., \& Stevens, C. K. (2006). Making sense of diversity in the workplace: Organizational justice and language abstraction in employees' accounts of diversity related incidents. Journal of Applied Psychology, 91, 379-391.

Rotundo, M., Nguyen, D. H., \& Sackett, P. R. (2001). A Meta-analytic review of gender differences in perceptions of sexual harassment. Journal of Applied Psychology, 86, 914-922.

Rossette, A. S., Leonardelli, G. J., \& Phillips, K. W. (2008). The White standard: Racial bias in leader categorization. Journal of Applied Psychology, 93, 758-777. 
Runco, M. A., \& Chand, I. (1995). Cognition and creativity. Educational Psychology Review, 7, 243-267.

Rupp, D. E., Bashshur, M. \& Liao, H. (2007). Justice climate past, present, and future: models of structure and emergence. In F. Dansereau, \& F. J. Yammarino (Eds.), Research In Multilevel Issues (Vol. 6). Englewood Cliffs, NJ: Erlbaum.

Rynes, S. \& Rosen, B. (1995). A field survey of factors affecting the adoption and perceived success of diversity training. Personnel Psychology, 48, 247-270.

Saucier, G. (1994). Mini-markers: A brief version of Goldberg's unipolar Big-Five markers. Journal of Personality Assessment, 63, 506-516.

Schaubroeck, J., Lam, S. S. K., \& Cha, S. E. (2007). Embracing transformational leadership: Team values and the impact of leader behavior on team performance. Journal of Applied Psychology, 92, 1020-1030.

Schneider, B. (1987). The people make the place. Personnel Psychology, 40, 437-453.

Schneider, B., Goldstein, H. W., \& Smith, D. B. (1995). The ASA framework: An update. Personnel Psychology, 48, 747-773.

Schepers, P. \& van den Berg, P. T. (2007). Social Factors of Work-Environment Creativity. Journal of Business and Psychology, 21 (3), 407-428.

Schumpeter, J. (1939). Business cycles: A theoretical, historical, and statistical analysis of the capitalist process. New York: McGraw-Hill.

Scullion, H., \& Collings, D. (2011). Global Talent Management. New York and London: Routledge. 
Seibert, S., Crant, M., \& Kraimer, M. (1999). Proactive personality and career success, Journal of Applied Psychology, 84, 416-427.

Shalley, C. E. (1991). Effects of productivity goals, creativity goals, and personal discretion on individual creativity. Journal of Applied Psychology, 76, 179-185.

Shalley, C. E. (1995). Effects of coaction, expected evaluation, and goal setting on creativity and productivity. Academy of Management Journal, 38, 483-503.

Shalley, C. E., \& Gilson, L. L. (2004). What leaders need to know: A review of social and contextual factors that can foster or hinder creativity. Leadership Quarterly, $15,33-53$.

Shalley, C. E., Gilson, L. L., \& Blum, T. C. (2000). Matching creativity requirements and the work environment: Effects of satisfaction and intention to leave. Academy of Management Journal, 43, 215-223.

Shalley, C. E., Gilson, L. L., \& Blum, T. C. (2009). Interactive effects of growth need strength, work context, and job complexity on self-reported creative performance. Academy of Management Journal, 52, 489-505.

Shalley, C. E., \& Perry-Smith, J. E. (2001). Effects of social-psychological factors on creative performance: The role of informational and controlling expected evaluation and modeling experience. Organizational Behavior and Human Decision Processes, 84, 1-22.

Shalley, C. E., Zhou, J., \& Oldham, G.R. (2004). The effects of personal and contextual characteristics on creativity: Where should we go from here? Journal of Management, 30(6), 933-958. 
Shao, L., \& Webber, S. (2006). A cross-cultural test of the 'five-factor model of personality and transformational leadership.' Journal of Business Research, 59, 936-944.

Shaw, M. E. (1981). Group dynamics: The psychology of small group behavior (3rd ed.). New York: McGraw-Hill.

Shin (2012). Cognitive team diversity and individual team member creativity: A crosslevel interaction. The Academy of Management Review, 37(2), 197.

Shin, S., \& Zhou, J. (2003). Transformational leadership, conservation, and creativity: Evidence from Korea. Academy of Management Journal, 46, 703-714.

Si, S., \& Wei, F. (2012). Transformational and transactional leaderships, empowerment climate, and innovation performance: A multilevel analysis in the Chinese context. European Journal of Work and Organizational Psychology, 21: 2, 299320.

Simonton, D. K. (1995). Foresight in insight? A Darwinian Answer. In R. J. Sternberg and J. E. Davidson (eds.) The nature of insight (pp. 465-494. Cambridge, MA: MIT Press.

Simonton, D. K. (1999). Creativity as blind variation and selective retention: Is the creative process Darwinian? Psychological Inquiry, 10, 309-328.

Singer, M. S., \& Singer, A. E. (1990). Situational constraints on transformational versus transactional leadership behaviour, subordinates' leadership preference, and satisfaction. Journal of Social Psychology, 130, 385-396. 
Singh, B., Winkel, D. E., \& Selvarajan, T. T. (2013). Managing diversity at work: Does psychological safety hold the key to racial differences in employee performance? Journal of Occupational and Organizational Psychology, 86, 242-263.

Smith, S. M., Ward, T. B., \& Finke, R. A. (Eds.). (1995). The creative cognition approach. Cambridge, MA: MIT Press.

Snijders, T. A. B., \& Bosker, R. (1999). Multilevel analysis: an introduction to basic \& advanced multilevel modelling. Sage Publications, London (1999).

Somech, A. (2006). The effects of leadership style and team process on performance and innovation in functionally heterogeneous teams. Journal of Management, 32, $132-157$.

Stewart, G. L. (2006). A meta-analytic review of relationships between team design features and team performance. Journal of Management, 32, 29-54.

Stockdale, M. S. \& Cao, C. (2004). Looking back and heading forward: Major themes of the psychology and management of workplace diversity. In M. S. Stockdale and F. J. Crosby (eds.), The psychology and management of workplace diversity (pp. 300-316). Malden, MA: Blackwell.

Szymanski, K., \& Harkins, S. G. (1992). Self-evaluation and creativity. Personality and Social Psychology Bulletin, 18, 259-265.

Tajfel, H., \& Turner, J. C. (1979). An integrative theory of intergroup conflict. In W. G. Austin \& S. Worchel (Eds.), Social psychology of intergroup relations (pp. 3347). Monterey, CA: Brooks-Cole. 
Tajfel, H., \& Turner, J. C. (1985). The social identity theory of intergroup behavior. In S. Worchel \& W. G. Austin (Eds.), Psychology of intergroup relations (2nd ed., pp. 7-24). Chicago: Nelson-Hall.

Taras et al (2010). A three-decade, multilevel, meta-analytic review of Hofstede's cultural value dimensions.

Taylor, A. S., James, K., \& Murry, A. (2012). Leveraging workforce diversity using a multidimensional approach. In C. L. Scott \& M. Y. Byrd (Eds.), Handbook of Research on Workforce Diversity and in a Global Society: Technologies and Concepts (pp. 203-224). IGI Global. Taxonomy. 2012. In dictionary.com. Retrieved September 28, 2012, from http://dictionary.reference.com/browse/taxonomy.

Taylor, P. J., Russ-Eft, D. F., \& Chan, D. W. L. (2005). A meta-analytic review of behavior modeling training. Journal of Applied Psychology, 90, 692-709.

Tepper, B. J., \& Percy, P. M. (1994). Structural validity of the Multifactor Leadership Questionnaire. Educational and Psychological Measurement, 54, 734-744.

Tesluk, P. E, Farr, J. L. \& Klein, S. R. (1997). Influences of organizational culture and climate on individual creativity. Journal of Creative Behavior, 31, 2741.

Thatcher, S. M. B., \& Greer, L. L. (2008). Does it really matter if you recognize who I am? The implications of identity comprehension for individuals in work teams. Journal of Management, 34, 5-24. 
Tierney, P., \& Farmer, S. M. (2002). Creative self-efficacy: Potential antecedents and relationship to creative performance. Academy of Management Journal, 45, 1137-1148.

Tierney, P., \& Farmer, S. M. (2004). The Pygmalion process and employee creativity. Journal of Management, 30, 413-432.

Tierney, P., Farmer, S. M., \& Graen, G. B. (1999). An examination of leadership and employee creativity: The relevance of traits and relationships. Personnel Psychology, 52, 591-620.

Tjosvold, D. (2008). The conflict-positive organization: It depends upon us. Journal of Organizational Behavior, 29, 19-28.

Torrance, E. P. (1974). Torrance tests of creative thinking: Directions manual and scoring guide. Englewood Cliffs, NJ: Prentice Hall.

Triandis, H. C. (2003). The Future of Workforce Diversity in International Organisations: A Commentary. Applied Psychology: An International Review, 52, 486-495.

Tsui, A. S., Egan, T. D., \& O’Reilly, C. A., III. (1992). Being different: Relational demography and organizational attachment. Administrative Science Quarterly, 37, 549-579.

Turner, J. C. (1981). The experimental social psychology of inter-group behavior. In J. C. Turner \& H. Giles (Eds.), Intergroup behavior (pp. 66-101). Chicago, IL: University of Chicago Press. 
Turner, J. C., \& Haslam, A. (2001). Social identity, organizations and leadership. In M. Turner (Ed.), Groups at work: Theory and research (pp. 25-65). Mahwah, NJ: Erlbaum.

van Dijk, H., van Engen, M. L.,\&van Knippenberg, D. (2012). Defying conventional wisdom: A meta-analytical examination of the differences between demographic and job-related diversity relationships with performance. Organizational Behavior and Human Decision Processes, 119, $38-53$.

van Knippenberg, D., De Cremer, D., \& van Knippenberg, B. (2007). Leadership and fairness: The state of the art. European Journal of Work and Organizational Psychology, 16, 113-140.

van Knippenberg, D., \& Schippers, M. C. (2007). Work group diversity. Annual Review of Psychology, 58, 515-541.

Ward, T. B., Smith, S. M., \& Finke, R. A. (1999). Creative cognition. J. Sternberg (Ed.), Handbook of creativity (pp. 189-212). New York: Cambridge University Press.

Wasserman, I. C., Gallegos, P. V., \& Ferdman, B. M. (2008). Dancing with resistance: Leadership challenges in fostering a culture of inclusion. In K. M. Thomas (Ed.), Diversity resistance in organizations: Manifestations and solutions (pp. 175-200). New York: Taylor \& Francis. 
Webber, S. S., \& Donahue, L. M. (2001). Impact of highly and less job-related diversity on work group cohesion and performance. Journal of Management, 27, 141-162.

Wegge, J., Roth, C., Kanfer, R., Neubach, B., \& Schmitt, K.-H. (2008). Age and gender diversity as determinants of performance and health in a public organization: The role of task complexity and group size. Journal of Applied Psychology, 93, 1301-1303.

Weisberg, R. W. (1993). Creativity: Beyond the myth of genius. New York: Freeman.

Wieland, A. J. (2004). Business leadership and diversity: The relationships between team diversity climate, leaders' diversity attitudes, and leadership styles. Dissertation Abstracts International: Section B: The Sciences and Engineering. Vol.65(4-B),2004, pp. 2134.

West, M. A., Tjosvold, D., \& Smith, K. (Eds.) (2003). International handbook of organizational teamwork and cooperative working (pp. 277-296) New York: Wiley.

Wiersema, M. F., \& Bantel, K. A. (1992). Top management team demography and corporate strategic change. Academy of Management Journal, 35, 91-121.

Williams, K., \& O’Reilly, C. A. (1998). Demography and diversity in organizations: A review of 40 years of research. In B. M. Staw \& L. L. Cummings (Eds.), Research in organizational behavior, 20, 77-140. Greenwich, CT: JAI Press. 
Wood, W. (1987). Meta-analytic review of sex differences in group performance. Psychological Bulletin, 102, 53-71.

Woodman, R. W., Sawyer, J. E., \& Griffin, R. W. (1993). Toward a theory of organizational creativity. Academy of Management Review, 18, 293-321.

Yuan, F., \& Zhou, J. (2008). Differential effects of expected external evaluation on different parts of the creative idea production process and on final product creativity. Creativity Research Journal, 20, 391-403.

Zaccaro, S. J., \& Banks, D. J. (2001). Leadership, vision, and organizational effectiveness. In S. J. Zaccaro \& R. J. Klimoski (Eds.), The nature of organizational leadership (pp. 181-218). San Francisco, CA: Jossey-Bass.

Zenger, T. R., \& Lawrence, B. S. (1989). Organizational demography: The differential effects of age and tenure distributions on technical communication. Academy of Management Journal, 32, 353-376.

Zhou, J. (1998). Feedback valence, feedback style, task autonomy, and achievement orientation: Interactive effects on creative performance. Journal of Applied Psychology, 83, 261-276.

Zhou, J., \& George, J. M. (2001). When job dissatisfaction leads to creativity: Encouraging the expression of voice. Academy of Management Journal, 44, $682-696$.

Zhou, J., \& Oldham, G. R. (2001). Enhancing creative performance: Effects of expected developmental assessment strategies and creative personality. Journal of Creative Behavior, 35, 151-167. 
Zhou, J., \& Shalley, C. E. (2003). Research on employee creativity: A critical review and directions for future research. In J. Martocchio (Ed.), Research in personnel and human resource management (pp. 165-217). Oxford, England: Elsevier.

Zhou, J., \& Shalley, C. E. (Eds.). (2008). Handbook of organizational creativity. New York: Erlbaum.

Zhou, J., \& Shalley, C. E. (2011). Deepening our understanding of creativity in the workplace: A review of different approaches to creativity research. In S. Zedeck (Ed.), APA handbook of industrial and organizational psychology, Vol. 1: Building and developing the organization (pp. 275-302). APA Handbooks in Psychology, Washington, DC, US: American Psychological Association.

Zhou, L. \& Shi, J. (2009). Psychometric properties of the Chinese translation of the proactive personality scale. Psychological Reports (105), 43-56. DOI 10.2466/PR0.105.1.43-56.

Zhou, J., Shin, S. J., \& Cannella, A. (2008). Employee self-perceived creativity after mergers and acquisitions: Interactive effects of threat-opportunity perception, access to resources, and support for creativity. Journal of Applied Behavioral Science, 44, 397-421.

Zhu, W., Newman, A., Miao, Q., \& Hooke, A. (2012). Revisiting the mediating role of trust in transformational leadership effects: Do different types of trust make a difference? 


\section{Transformational Leadership}

Participants were asked to respond based on the following 7-point scale:

$1=$ Strongly Disagree

2 = Moderately Disagree

3 = Slightly Disagree

$4=$ Neutral

5 = Slightly Agree

$6=$ Moderately Agree

$7=$ Strongly Agree

Please indicate your level of agreement or disagreement with each statement by circling one of the seven alternatives next to each statement.

I believe my supervisor...

\section{Inspirational Motivation}

1. Seeks new opportunities for our organization.

2. Paints an interesting picture of the future for our work group.

3. Has a clear understanding of where we are going.

4. Inspires others with his/her plans for the future.

5. Is able to get others to commit to his/her dream(s) for the future.

\section{Intellectual Stimulation}

6. Provides individuals with new ways of looking at things that are puzzling to them.

7. Has ideas that have forced individuals to rethink some of their own ideas.

8. Stimulates individuals to think about old problems in new ways.

9. Is good at getting individuals to think "outside the box".

10. Helps individuals be creative when difficult problems arise. 


\section{Workplace Diversity}

Participants were asked to respond based on the following 7-point scale:

$1=$ Strongly Disagree

2 = Moderately Disagree

3 = Slightly Disagree

$4=$ Neutral

$5=$ Slightly Agree

$6=$ Moderately Agree

$7=$ Strongly Agree

Please indicate your level of agreement or disagreement with each statement by circling one of the seven alternatives next to each statement.

\section{Diversity Climate}

1. Senior management is committed to diversity in my organization.

2. My organization takes steps to increase diversity.

3. Organization policies support my manager in increasing diversity.

4. My organization puts a lot of resources into diversity initiatives.

5. Leaders here connect diversity to the organization's mission and vision.

\section{Organizational Justice}

6. People at work are treated fairly regardless of who they are.

7. Policies are implemented consistently for all employees.

8. My manager creates a comfortable working environment for all types of people.

9. Certain people are denied opportunities at work because of who they are. $(R)$

\section{Organizational Identity}

10. I consider myself part of my work team.

11. Ifeel separate from my co-workers. (R)

12. I identify with my co-workers.

13. I feel a strong sense of belonging at my organization.

Note. Items in italics were deleted from the composite variables used in path analyses. 


\section{Employee Self-rated Individual Creative Performance}

Participants were asked to respond based on the following 7-point scale:

$1=$ Strongly Characteristic

$2=$ Moderately Characteristic

3 = Slightly Characteristic

$4=$ Neutral

5 = Slightly Uncharacteristic

$6=$ Moderately Uncharacteristic

7 = Strongly Uncharacteristic

Please indicate how characteristic each behavior is of your actions at work by circling one of the seven alternatives next to each statement.

1. I suggest new ways to achieve goals or objectives.

2. I come up with new and practical ideas to improve performance.

3. I seek out new technologies, processes, techniques, and/or product ideas.

4. I suggest new ways to increase quality.

5. I am a good source of creative ideas.

6. I am not afraid to take risks.

7. I promote and champion ideas to others.

8. I exhibit creativity on the job when given the opportunity to.

9. I develop adequate plans and schedules for the implementation of new ideas.

10. I often have new and innovative ideas.

11. I come up with creative solutions to problems.

12. I often have a fresh approach to problems.

13. I suggest new ways of performing work tasks. 


\section{Supervisor-rated Individual Creative Performance}

Participants were asked to respond based on the following 7-point scale:

1 = Strongly Characteristic

$2=$ Moderately Characteristic

3 = Slightly Characteristic

$4=$ Neutral

5 = Slightly Uncharacteristic

$6=$ Moderately Uncharacteristic

7 = Strongly Uncharacteristic

Please indicate how characteristic each behavior is of your actions at work by circling one of the seven alternatives next to each statement.

\section{The employee who I supervise...}

1. $\quad$ suggests new ways to achieve goals or objectives.

2. comes up with new and practical ideas to improve performance.

3. seeks out new technologies, processes, techniques, and/or product ideas.

4. suggests new ways to increase quality.

5. is a good source of creative ideas.

6. is not afraid to take risks.

7. promotes and champions ideas to others.

8. exhibits creativity on the job when given the opportunity to.

9. develops adequate plans and schedules for the implementation of new ideas.

10. often has new and innovative ideas.

11. comes up with creative solutions to problems.

12. often has a fresh approach to problems.

13. suggests new ways of performing work tasks.

Note. Items in italics are did not perform statistically as well as the other items in the scale. However, all items were retained and included in the composite variable used in the path analyses. 


\section{Proactive Personality}

Participants were asked to respond based on the following 7-point scale:

$1=$ Strongly Disagree

$2=$ Moderately Disagree

3 = Slightly Disagree

$4=$ Neutral

$5=$ Slightly Agree

$6=$ Moderately Agree

$7=$ Strongly Agree

Please indicate your level of agreement or disagreement with each statement by circling one of the seven alternatives next to each statement.

1. I am constantly on the lookout for new ways to improve my life.

2. Wherever I have been, I have been a powerful force for constructive change.

3. Nothing is more exciting than seeing my ideas turn into reality.

4. If I see something I don't like, I fix it.

5. No matter what the odds, if I believe in something, I will make it happen.

6. I love being a champion of my ideas, even against others' opposition.

7. I excel at identifying opportunities.

8. I am always looking for better ways to do things.

9. If I believe in an idea, no obstacle will prevent me from making it happen.

10. I can spot a good opportunity long before others can. 


\section{Openness to Experience}

Participants were asked to respond based on the following 7-point scale:

$1=$ Strongly Disagree

2 = Moderately Disagree

3 = Slightly Disagree

$4=$ Neutral

5 = Slightly Agree

$6=$ Moderately Agree

7 = Strongly Agree

Please indicate your level of agreement or disagreement with each statement by circling one of the seven alternatives next to each statement.

At work, I tend to be...

1. Philosophical

2. Complex

3. Uncreative

4. Imaginative

5. Deep

6. Unintellectual

7. Creative

8. Intellectual

Note. The openness to experience composite variable analyzed in the path analyses was composed of the items in bold. 